Florida International University FIU Digital Commons

\title{
Secure power grid infrastructure simulation and test-bed system creation
}

Nelson Cristaldo

Florida International University

DOI: $10.25148 /$ etd.FI14061543

Follow this and additional works at: https://digitalcommons.fiu.edu/etd

Part of the Electrical and Computer Engineering Commons

\section{Recommended Citation}

Cristaldo, Nelson, "Secure power grid infrastructure simulation and test-bed system creation" (2007). FIU Electronic Theses and Dissertations. 2668.

https://digitalcommons.fiu.edu/etd/2668 


\section{FLORIDA INTERNATIONAL UNIVERSITY}

Miami, Florida

SECURE POWER GRID INFRASTRUCTURE SIMULATION AND

TEST-BED SYSTEM CREATION

A thesis submitted in partial fulfillment of the

requirements for the degree of

MASTER OF SCIENCE

in

ELECTRICAL ENGINEERING

by

Nelson Cristaldo 
To: Dean Vish Prasad

College of Engineering and Computing

This thesis, written by Nelson Cristaldo, and entitled Secure Power Grid Infrastructure Simulation and Test-bed System Creation, having been approved in respect to style and intellectual content, is referred to you for judgment.

We have read this thesis and recommend that it be approved.

Nikki Pissinou

Jean Andrian

Osama Mohammed, Major Professor

Date of Defense: July 19, 2007

The thesis of Nelson Cristaldo is approved.

Dean Vish Prasad College of Engineering and Computing

Dean George Walker University Graduate School

Florida International University, 2007 


\section{DEDICATION}

I dedicate this work to my parents Porfirio and Stella. 


\section{ACKNOWLEDGMENTS}

I would like to express my deepest gratitude to my major professor, Dr. Osama Mohammed, for providing me the significant opportunity to work on this project partially supported by the U.S. Department of Energy. Dr. Mohammed ideas on Secure Power Grid Infrastructure are the foundation of this work and his principles on local diagnosis and distributed control systems form the backbone of the implementation examples presented in this thesis.

I would like to give special thanks to my friends and colleagues, Dr. Nagy Abed, Dr. Zhiquiang Liu, Dr. Shreerang Ganu, Dr. Shou Liu and Mr. Luis Henao who provided me with unconditional help to solve the problems I faced while working with the thesis. Their support will be always remembered and treasured.

I would also like to thanks to my graduate committee members Dr. Jean Andrian and Dr. Pissinou for reviewing this work and for their support. 
ABSTRACT OF THE THESIS

SECURE POWER GRID INFRASTRUCTURE SIMULATION AND

TEST-BED SYSTEM CREATION

by

Nelson Cristaldo

Florida International University, 2007

Miami, Florida

Professor Osama Mohammed, Major Professor

This thesis describes the design and construction of the test-bed system as well as its implementation to simulate the distributed control scheme. Some important elements of the system incorporate flexible power system network hardware and a data acquisition (DAQ) system to evaluate the issues that could trigger cascaded failures.

The objective of this research was to create an integrated simulation environment that represents the power system devices, the control, the protection, and the communication subsystems in the same simulation environment. We have utilized this simulator to conduct system studies and evaluate the interaction between power system components under steady-state and transient operation. The results of these studies have been used to enhance the system's ability to identify, and respond to the system's dynamic transients; improving the survivability and robustness of the power system during and after a disturbance by utilizing distributed control strategies. Experiments demonstrated in this thesis show the applicability of the developed simulator to conduct system studies and to evaluate the proposed distributed control infrastructure. The results show the advantages of utilizing distributed control and the impact in the system security and reliability. 


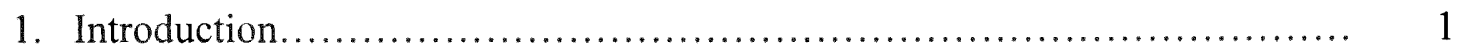

1.1. Literature Review................................................. 1

1.2. Problem Statement................................................... 4

1.3. Contributions of this Research to the Current State of Art................ 7

1.4. Organization of Thesis............................................. 9

2. Overview of the Current State of Power System Operations.................. 10

2.1. Power System Operation............................................... 10

2.2. Real-Time Sequence of Operation................................... 13

2.3. The Dynamic Control Problem..................................... 16

3. Distributed Control Infrastructure.......................................... 18

3.1. Distributed Control and Software Agents.............................. 18

3.2. Distributed Control Infrastructure Solution for DCP...................... 19

3.3. Distributed Control Infrastructure in Power System Networks............ 20

4. Telecommunication Network Infrastructure .............................. 22

4.1. Distributed Control Infrastructure and Telecommunication Network...... 22

4.2. Telecommunication Network Methods of Implementation................. 23

5. Application of Power Electronics Processor as Distributed Agents............ 27

5.1. Power Electronics and Flexible AC Transmission Systems (FACTS)...... 27

5.2. Application of FACTS Devices as Distributed Agents................... 28

5.3. Thyristor Switch Braking Resistor (TSBR) ......................... 29

5.4. Real Time Distributed Control Implementation for the TSBR........... 34

6. Hardware and Software Integration for the Real-Time Control System... 38

6.1. Introduction...................................................... 38

6.2. Block Diagram and Schematic Interface........................... 39

6.3. Real Time System Configuration..................................... 41

7. Test-bed System Development and Integration............................ 43

7.1. Introduction..................................................... 43

7.2. System Description and Scaling................................... 44

7.3. Hardware Sub-System ........................................... 46

7.3.1. Test-Bed Equipment.......................................... 46

7.3.2. Power Electronic Converter Board............................. 51

7.3.2.1. Thyristor Switch Braking Resistor (TSBR) Model......... 53

7.3.3. Development of the Transmission Line Model..................... 55

7.3.4. Development of the Bus-Bar Model............................ 58

7.3.5. Physical Model of the Overall Test-Bed........................... 60 
8. System Implementation and Experimental Results.............................................. 65

8.1. Transient Stability Problem........................................... 65

8.2. Case of Studies and System Performance Evaluation..................... 66

8.2.1. Two-Bus Test System Example.................................. 67

8.2.2. Four-Bus Test System Example................................ $\quad 70$

8.2.3. Classical 9-Bus Standard Test System Example..................... 73

8.2.3.1. The 9-Bus System Under Unexpected Load Variation Example.................................................... 74

8.2.3.2. The 9-Bus System Undergoes a Three-Phase Fault Example....

9. Discussion and Results ............................................... 81

10. Recommendations and Future Work .................................... 82

REFERENCES.............................................................. 83

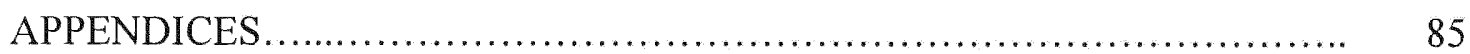




\section{LIST OF FIGURES}

FIGURE

PAGE

1.2.1 Centralized control infrastructure ............................ 5

1.2.2 Distributed control infrastructure ............................. 7

2.1.1 Profile of the power system operation.......................... 12

2.2.1 State-of-the art architecture of an EMS at the control center....... 13

2.2.2 Security assessment for stability limits of the power system........ 14

3.1.1 Multilevel distributed control system........................... 19

3.2.1 An illustration of the distributed control solution for DCP ........ 20

4.1.1 Collaborative and telecommunication network .................. 23

4.2.1 The communication setup for the 9-bus with two FACTS devices. 24

4.2.2 The central control data acquisition system...................... 25

4.2.3 Remote terminal units (RTUs) display....................... 26

4.2.4 Master station display ....................................... 26

5.1.1 Block diagram of a power electronic processor.................... 28

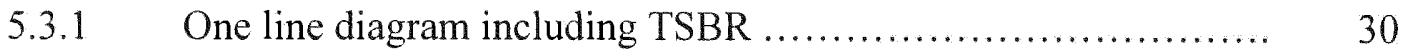

5.3.2 Wye and delta configuration for the TSBR.................... 31

5.3.3 Power angle diagram without TSBR .......................... 32

5.3.4 Power angle diagram with TSBR ............................. 33

5.3.5 Operation for damping oscillation and dynamic instability......... 33

5.4.1 Real-time control block diagram for the TSBR ................... 35

5.4.2 Real-time distributed control implementation for TSBR devices... 35

5.4.3 Matlab blocks for the PID controller........................... 36 
6.2.1 Host-target real time software subsystems architecture........... 40

6.3.1 Configuration of the real time simulator......................... 42

7.1.1 Power system four main subsystems.......................... 44

7.2.1 Scheme for components' blocks................................ 45

7.3.1.1 Supply and metering console workstation ...................... 47

7.3.1.2 Motor-generator set-up.................................... 48

7.3.1.3 Three-phase transformer...................................... 49

7.3.1.4 Resistance-reactance load .................................. 49

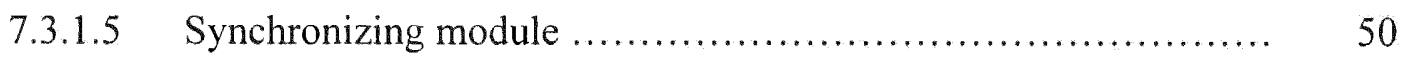

7.3.1.6 Resistance load cart ......................................... 51

7.3.2.1 Power electronic converter board ............................... 52

7.3.2.2 Circuit layout for the converter ................................ 53

7.3.2.1.1 Layout of the TSBR attached to the generator bus................. 54

7.3.2.1.2 Schematic of the (RTS) and the command station............... 55

7.3.3.1 Prototype schematic for the transmission line model .............. 56

7.3.3.2 Transmission line model for 150 miles.......................... 56

7.3.3.3 Transmission line model for 70 miles............................ 57

7.3.4.1 Prototype model for the bus-bar............................ 58

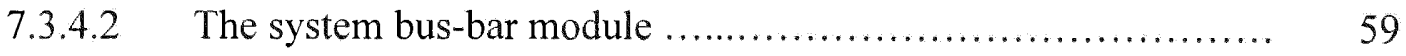

7.3.5.1 Two-bus test system.......................................... 60

7.3.5.2 One-line diagram for the two-bus test system.................... 61

7.3.5.3 Four-bus test system.................................... 62 
7.3.5.4 One-line diagram for the four-bus test system................. 62

7.3.5.5 Modify IEEE 9-bus multimachine test system................... 63

7.3.5.6 One-line configuration for the 9-bus test system .................. 64

8.2.1 Procedures to assess transient stability problems ............... 67

8.2.1.1 The two-bus test system during the event...................... 68

8.2.1.2 Frequency profile before, during and after the line tripped........ 69

8.2.1.3 Frequency response of the system with control action............. 69

8.2.2.1 The four-bus test system during the three-phase fault at Bus_3... 71

8.2.2.2 The system frequency collapses and triggered a Blackout......... 71

8.2.2.3 The distributed control infrastructure solving the DCP........... 72

8.2.2.4 The load rms current recovering to normal operational mode...... 73

8.2.3.1.1 The 9-bus test with an unexpected load variation at Bus 2........ 74

8.2.3.1.2 The frequency profile increasing after the load variation.......... 75

8.2.3.1.3 The frequency is controlled by the distributed control agents...... 76

8.2.3.2.1 The 9-bus one-line diagram during the three phase fault at Bus_3. 77

8.2.3.2.2 The frequency profile without the distributed control agents....... 78

8.2.3.2.3 The frequency profile with distributed control agents............. 78

8.2.3.2.4 The rms voltage profile without the distributed control action....... 79

8.2.3.2.5 The rms voltage profile with the distributed control action......... 79 


\section{LIST OF ACRONYMS}

NERC North America Electric Reliability Council

RTO Regional Transmission Organization

ISO Independent System Operator

SSSL Steady-State Stability Limits

VSL Voltage Stability Limits

TSL Transient Stability Limits

SCADA Supervisory Control and Data Acquisition System

FACTS Flexible AC Transmission System

DAQ Data Acquisition

ATC Available Transfer Capability

TTC Total Transfer Capability

DCP Dynamic Control Problems

IP Internet Protocol

TCP Transmission Control Protocol

TSBR Thyristor Switch Braking Resistor

HIL Hardware in the Loop

FPGA Field Programmable Gate Array

RTW Real Time Workshop

RTU Remote Terminal Unit

RTS Real-Time Station

PC Personal Computer 


$\begin{array}{ll}\text { Ag } & \text { Agent } \\ \mathrm{T}_{\mathrm{a}} & \text { Accelerating Torque } \\ \mathrm{T}_{\mathrm{m}} & \text { Mechanical Driving Torque } \\ \mathrm{T}_{\mathrm{e}} & \text { Electrical or Load Torque } \\ \dot{\delta} & \text { Rotor Angle of the Generator } \\ \omega & \text { Rotor Speed Deviation } \\ \omega_{0} & \text { Initial Speed of the Generator } \\ \mathrm{H} & \text { Deffective Inertia of the Generator } \\ \mathrm{D} & \text { Electric Power Injection from the Generator } \\ \mathrm{Pe} & \text { Mechanical Power of the Generator } \\ \mathrm{Pm} & \text { Maximum Clearing Angle } \\ \delta_{\text {max }} & \text { Voltage at Bus 1 } \\ \mathrm{E}_{1} & \text { Reactance of the Line } \\ \mathrm{X} & \end{array}$




\section{Chapter 1}

\section{Introduction}

\subsection{Literature Review}

Cascading failures in power system networks cause blackouts, which often lead to severe social and economic consequences. Cascading failures are usually initiated by a set of equipment failures that cause operating limit violations.

It is often very difficult to understand what really causes a cascading failure; although some general indications are determined. For example; the probability of a cascading event increases as the transmission system loading increases [1].

A well know example of transmission overload that caused a cascading failure is the U.S. 1965 Northeastern blackout; where, a faulty relay setting on a line between Niagara and Toronto tripped. This occasioned the power to shift to three parallel lines, which rapidly became overloaded, triggering other relay actions. The excess generated power at Niagara was immediately sent to New York State, overloading additional lines and resulting in a cascaded failure [2]. The consequences of blackouts like this one and others can become very severe as shown in Table 1.1 [3].

Throughout the history of blackouts in the U.S., cascading failures have reshaped the power system regulation and technology. For example; the 1965 blackout resulted in the creation of the North American Electric Reliability Council (NERC). After the 1977 failure, engineers developed the "N-1" reliability criterion that requires systems to be operated so as to withstand all single contingencies (i.e. one line de-energized, one generator unit outage, line-ground short circuit); this reliability criterion was then adopted by the NERC. In the aftermath of the 2003 blackouts that affected the northeastern of the 
U.S. and other tied regions, renewed attention has been focused on maintaining the highest level of reliability and security in the power system operations response to these events.

Table 1.1 - Several large scale cascading failures (NERC)

\begin{tabular}{|c|c|c|}
\hline DATE & Location & Consequences \\
\hline 9 Nov. 1965 & $\begin{array}{l}\text { Northeaster U.S., } \\
\text { Ontario }\end{array}$ & $30,000,000$ customers $(20,000 \mathrm{MW})$ interrupted. \\
\hline 13 July 1977 & New York City & $\begin{array}{l}9,000,000 \text { customers }(6,000 \mathrm{MW}) \text { interrupted. } \\
\text { Widespread looting and chaos. Police made } 4000 \\
\text { arrests. }\end{array}$ \\
\hline 2 July 1996 & Western U.S. & $2,000,000$ customers $(11,850 \mathrm{MW})$ interrupted. \\
\hline 3 July 1996 & Western U.S. & The disturbance from July 2 reoccurred. \\
\hline 10 Aug. 1996 & Western U.S. & $30,000,000$ customers $(20,000 \mathrm{MW})$ interrupted. \\
\hline 25 June 1998 & $\begin{array}{l}\text { Midwestern } \\
\text { U.S., central } \\
\text { Canada }\end{array}$ & $152,000$ customers ( $950 \mathrm{MW})$ interrupted. \\
\hline $\begin{array}{l}\text { Nov. } 1988 \text { to } \\
\text { June } 2003\end{array}$ & Western India & $\begin{array}{l}29 \text { large cascading failures over } 15 \text { years- }-1.9 \text { per } \\
\text { year. Millions of customers interrupted in most } \\
\text { cases. }\end{array}$ \\
\hline 14 Aug. 2003 & $\begin{array}{l}\text { Midwestern and } \\
\text { Northeastern US, } \\
\text { Southeastern } \\
\text { Canada } \\
\end{array}$ & $\begin{array}{l}50,000,000 \text { customers interrupted. Estimates of the } \\
\text { social costs range from } \$ 6 \text { billion (Graves, } 2003 \text { ) to } \\
\$ 10 \text { billion (ICF, 2003). Massive traffic jams in New } \\
\text { York City. }\end{array}$ \\
\hline 27 Sept. 2003 & Italy & $\begin{array}{l}57,000,000 \text { customers interrupted. At least } 5 \text { deaths } \\
\text { resulted. } 30,000 \text { passengers stranded in trains for } \\
\text { hours. }\end{array}$ \\
\hline
\end{tabular}

The most recent large scale failures in the electrical grid systems have revealed weaknesses in the control infrastructures, operating procedures and, in some cases, detailed understanding of the system dynamic response to an initial minor disturbance.

The results of these recent failures have reshaped many current ventures and redirected future focus of research in the broad area of electricity grid infrastructure development, 
insertion of new technologies to the grid, advanced control application, and increased reliability and security measures for the future electric system.

Some specific research focuses on this new trend are the development of custom microgrids, adaptive islanding, and integration of different types of distributed generation units into the electrical grid. Also the developments of high fidelity modeling for simulation and hardware in the loop, and the implementation of collaborative networks and intelligent agents in addition to the application of Flexibles AC Transmission System (FACTS) devices have become important.

The motivation for this thesis is based on the concept of Secure Power Grid Infrastructure proposed by Mohammed [4]. The research focused on the critical issues related to modernization and reliability enhancements of electric networks infrastructure.

Several research works by Talukdar and Caponogara [5-6] developed the mathematical structure and problem solving techniques for controlling networks with collaborative nets. Their work also includes the development of a framework for specifying the agents' tasks, the collaboration protocols, coordination layer, and agents' implementation in power system application.

The research work by Rehtanz, Handschin, and Becker [7-8] proposed the utilization of autonomous system and intelligent agents in the power system control and operation. They clearly exposed the infrastructure problems of centralized control and the advantages of distributed control infrastructure. The core of their work focuses on the design and application of control system architecture which provides and uses data and information on the various process levels appropriately. Also, their research included the development of the communication infrastructure technology required for the utilization of distributed 
control agents, the multi-agent negotiation models for power system applications. These authors also proposed the utilization of multi-agents approach to power system disturbance diagnosis, power system restoration, power system protection, coordination for secondary voltage control, and the development of a coordinating autonomous FACTS control systems.

This thesis proposes a method for limiting the costs of cascading failures by eliminating violation before dependent outages occurs. Specifically, the method developed here will create a hardware test-bed system to prove some of the concepts on distributed control infrastructures. This test-bed will help verify the applicability of FACTS devices as autonomous agents working in a coordinated network to control and mitigate transient stability problems that could trigger a cascaded event.

\subsection{Problem Statement}

In the past, the central control infrastructure was capable to preserve security and reliability of the power system because operations were planned and unscheduled transactions were uncommon. System analysis and corrective control actions were achieved by following the operating course of action based on off-line studies. Details on the current state of the art in power system operation are in chapter 2. In this chapter a summary of the standardized real-time sequence of operation as well as static and dynamic stability assessment methods in bulk control.

Nowadays, preserving security and reliability have become a very difficult task in a deregulated environment were very large and complex interconnected systems are subjected to unplanned fast changes. Even though determination of thermal limits and 
voltages violations are done on-line, system security and reliability are still based on offline system stability assessment [9].

Usually, the central control system effectively manages simultaneous conflicting objectives. For example, if a fault is created due to a lightning strike on a transmission line, the designed protection relays will trip the line to clear the fault and quickly restore the line to its operating mode. Here, the reliability and protection objectives were simultaneously achieved,

Central control infrastructure, shown in Figure (1.2.1), operates the electric power systems under very well defined system objectives, including:

- Economics - maximize the net economic benefit of service.

- Reliability - minimize the risk of service interruption.

- Protection-minimize the risk of infrastructure damage.

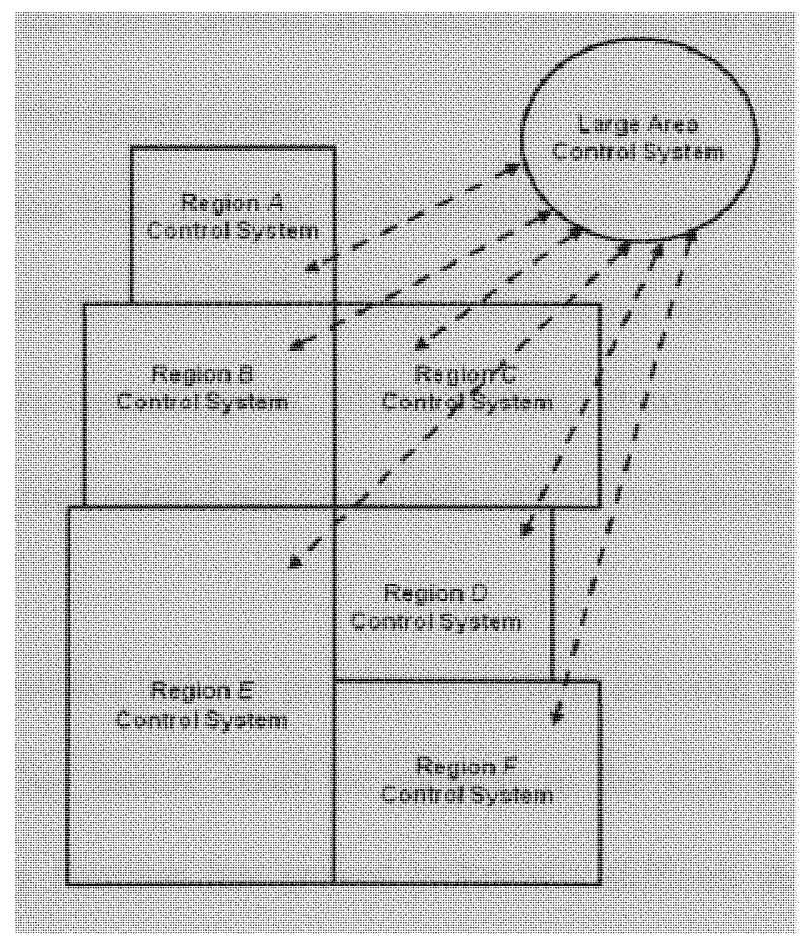

Figure (1.2.1) Centralized control infrastructure. 
On the contrary; centralized control systems do a very poor job balancing conflicting objectives during unexpected events. This is due to the fact that the central control infrastructure depends on the Supervisory Control and Data Acquisition (SCADA) Systems that does not predict nor respond to perturbations in the system. The system restoration depends on human operators (lack of real-time control systems). Even, aspiring to achieve real time control of the entire network from a central main frame location is highly improbable if not impossible. This is due to the complexity of power grid and the large amount of data computation. For example, only in the U.S. eastern interconnect there are approximately 100 control areas and about 50,000 buses controlled by hundreds of human operators, and thousands of instruments.

In other words, the central control infrastructure is not adequate to react effectively to local operating constraints violation or suppress it in time before causing a cascading failure. This is due to the size of system information involved, the lack of real-time control and the high inertia of the power system.

For this reason it has been proposed to decentralize the control of power networks in a distributed manner with the utilization of many autonomous agents. The proposed control scheme utilize software control agents; where, each agent solves its local problem using model predictive real-time control and cooperation within neighboring agents, as shown in Figure (1.2.2).

This thesis demonstrates that it is possible to perform network task without large scale centralized control. This feature could be valuable in the U.S. where centralization of control and regulatory function has proved technically difficult due to the free market operation of the power system. 


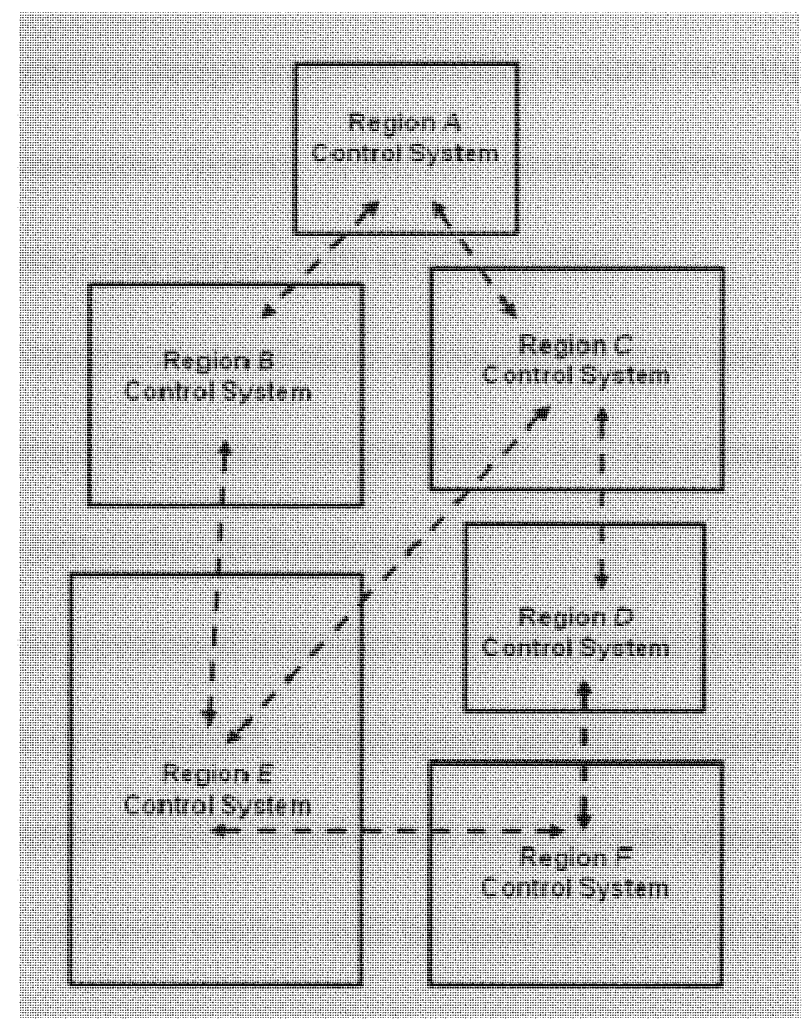

Figure (1.2.2) Distributed control infrastructure.

\subsection{Contributions of this Research to the Current State of the Art}

The specific goal of this research is to develop a test-bed system to prove that a distributed network infrastructure with autonomous agents is capable of eliminating power system violation before the protection system acts to disconnect equipment. For this method to be capable of increasing the over all reliability of the system without conflicting with other objectives; the agents must locally suppress violations, while maintaining everything else constant.

Essentially, the goal of this thesis is to concentrate on transient stability control aspects, and to implement the concepts of distributed monitoring, evaluation and control with system coordination. 
The methodology used to design transient stability control techniques relies on the use of Flexible AC Transmission System (FACTS) devices. Some of the most relevant contribution of this thesis to the current state of art includes:

- Create an integrated simulation environment that will represent the power system devices, the control, the protection, and the communication subsystems in the same simulation environment.

- To utilize the developed simulator to conduct system studies and evaluate the performance of distributed control agents and the interaction between the power system components under steady-state and transient operation.

- The results of these studies will be used to enhance the system's ability to identify, and respond to system's dynamic transients.

- Improve the survivability and robustness of the power system during and after a disturbance by utilizing distributed control strategies.

- This will increase the power system reliability and security under different operating conditions.

Finally, the development test-bed power system and the experimental results of implementing the distributed control infrastructure. This serves as a way to measure the performance of autonomous control for FACTS devices as well as demonstrate the benefits of a distributed control infrastructure in power system operation. The results would be the ability to mitigate a contingency that could trigger a cascaded event.

These are the important contribution of this work since it implies that it is possible to solve some global power network problems without the centralized control infrastructures 
and suggest the possibility to develop a self-monitoring, self-corrective and self-healing power grid.

\subsection{Organization of Thesis}

This thesis is organized in several additional chapters. Chapter 2 covers the power system operation utilizing the standard centralize control and the real-time sequence of operation. This chapter also covers the issues and disadvantages that centralized (bulk) control encounters while trying to solve dynamic control problems. Chapter 3 covers the concept of distributed control infrastructure and distributed control agents as well as its implementation into the power system network. In Chapter 4, the telecommunication network infrastructure is described and discussed in addition to the methods of implementation. This network infrastructure is required for the implementation of the distributed control infrastructure. Chapter 5 concentrates on the utilization of power electronic converters and the application of FACTS devices as distributed agents. Chapter 6 focuses on the application of real-time control implementation for these distributed agents. Chapter 7 discusses the development of the hardware test-bed system in the laboratory. This chapter also includes details on the design, scale, components, as well as software and hardware interfaces. Chapter 8 shows the distributed control experiments and their implementation of the integrated test-bed system. Chapter 9 gives a discussion of the results obtained by the implementation examples. Chapter 10 gives recommendations for future work. 


\section{Chapter 2}

\section{Overview of Current State of Power System Operations}

The electric utility industry is probably the largest and most complex industry in the world. With the present rise in demand of electrical energy, utility systems will continue to grow both in size and complexity. Also, our dependence on electricity is so great that it is essential to have an uninterrupted supply of power within set limits of frequency and voltage levels. Due to economic reasons and uncertainties in the forecast of loads, power reliability does not reach the $100 \%$ level. Consequently, the burden on system operators has increased tremendously in their effort to ensure that all customers are satisfied. Therefore, it becomes a necessity to evaluate new control system infrastructures; such as, the distributed control infrastructure.

\subsection{Power System Operation}

The power flow is strictly governed by the electric network equations. The flow pattern depends mainly on the load, the generation, distribution, and the network configuration. The amount of the power generated by each unit is constrained by its capacity. The rating of each line limits its flow of electric power, and so is the power handled by each transformer. For system security purposes, constraints may be imposed on the bus voltage angle across the lines. Voltages levels are to be within acceptable range. All of these conditions may be expressed in the form of equality constraints and the set of inequality constraints [10].

When the above constraints are satisfied, the power system is said to be in the normal operating state. Under such a condition, all the loads are met and all the system components are loaded within specified limits. It is the aim of the operator to maintain the system in the 
normal operating condition, even under expected circumstances. The controls chosen by the operator to achieve this aim are thus preventing in nature.

In practice, regional transmission organization (RTO) and independent system operators (ISOs), operate and coordinate the competitive wholesale electricity market. This ensures that the security and reliability from a centralized control and dispatch center are achieved. Federally regulated RTO are independent and impartial in managing the regional transmission system and the wholesale electricity market. RTO's associate members include power generators owners, transmission owners, electricity distributors, power marketers, and large consumers [10].

According to the U.S. Federal Energy Regulatory Commission (FERC), the RTO's goal is to promote efficiency in wholesale electricity markets and to ensure that electricity consumers pay the lowest price possible for reliable service. Listed below, is the FERC's definition of the minimum function than RTOs must satisfy:

- Tariff Administration and Design

- Congestion Management

- Parallel Path Flow

- Total Transmission Capability (TTC)

- Available Transmission Capability (ATC)

- Market Monitoring

- Planning and Expansion

- Interregional Coordination

Figure (2.1.1) shows the profile of the power system operation. The figure also show the centralized infrastructure (i.e. RTO, ISO); as well as its components, and 
characteristics. Furthermore, figure shows the operation of RTOs with each of them monitoring its region and delivering power at the lowest possible price while maintaining the highest possible system reliability.

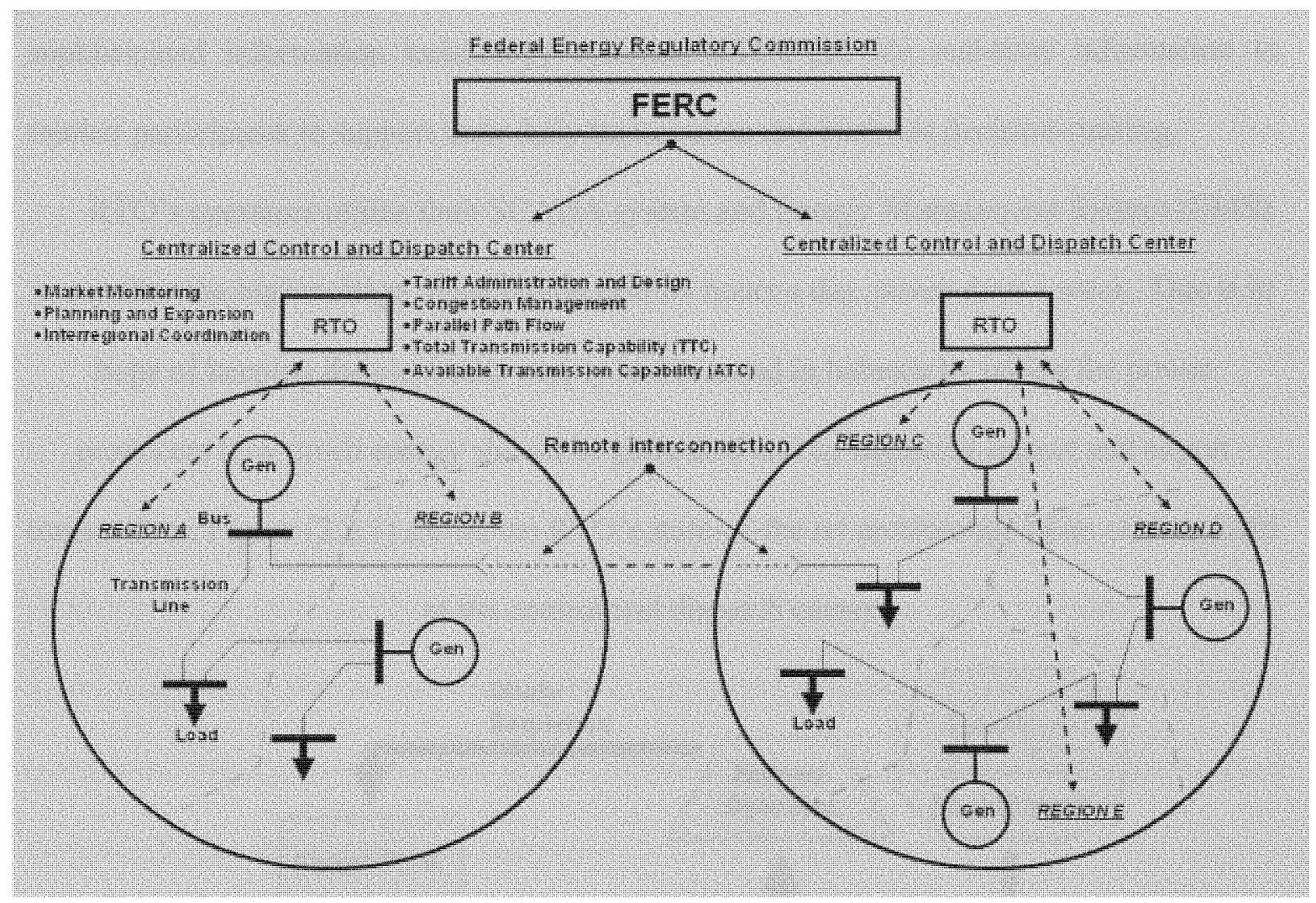

Figure (2.1.1) Profile of the power system operation.

RTOs are responsible for maintaining the integrity of the regional power grid and for managing changes and additions to the grid to accommodate new generating plants, substations and transmission lines. Also, they must analyze and forecast the future electricity needs of the region. The RTOs must ensure that the growth of the electric system takes place efficiently in an orderly planned fashion and that reliability is maintained. RTOs must develop innovative programs, such as demand-response initiatives and efforts to support renewable energy, to help expand supply options and keep prices competitive. 


\subsection{Real-Time Sequence of Operation}

To ensure the maximum reliability of the electric system, RTOs manage a sophisticated regional planning process for generation and transmission expansion. Utilizing sophisticated information technology systems, RTOs provide real-time information to market participants to support their daily transactions and business decision-making.

RTO's operators utilize their SCADA system and Energy Management System (EMS) to monitor the high-voltage transmission grid 24 hours a day, seven days a week. This is in hope of keeping the electricity supply and demand in balance by telling the power producers how much energy should be generated and by adjusting import and export transactions. Figure (2.2.1) shows the RTO's state-of-the art architecture of an on-line EMS at the control and dispatch center.

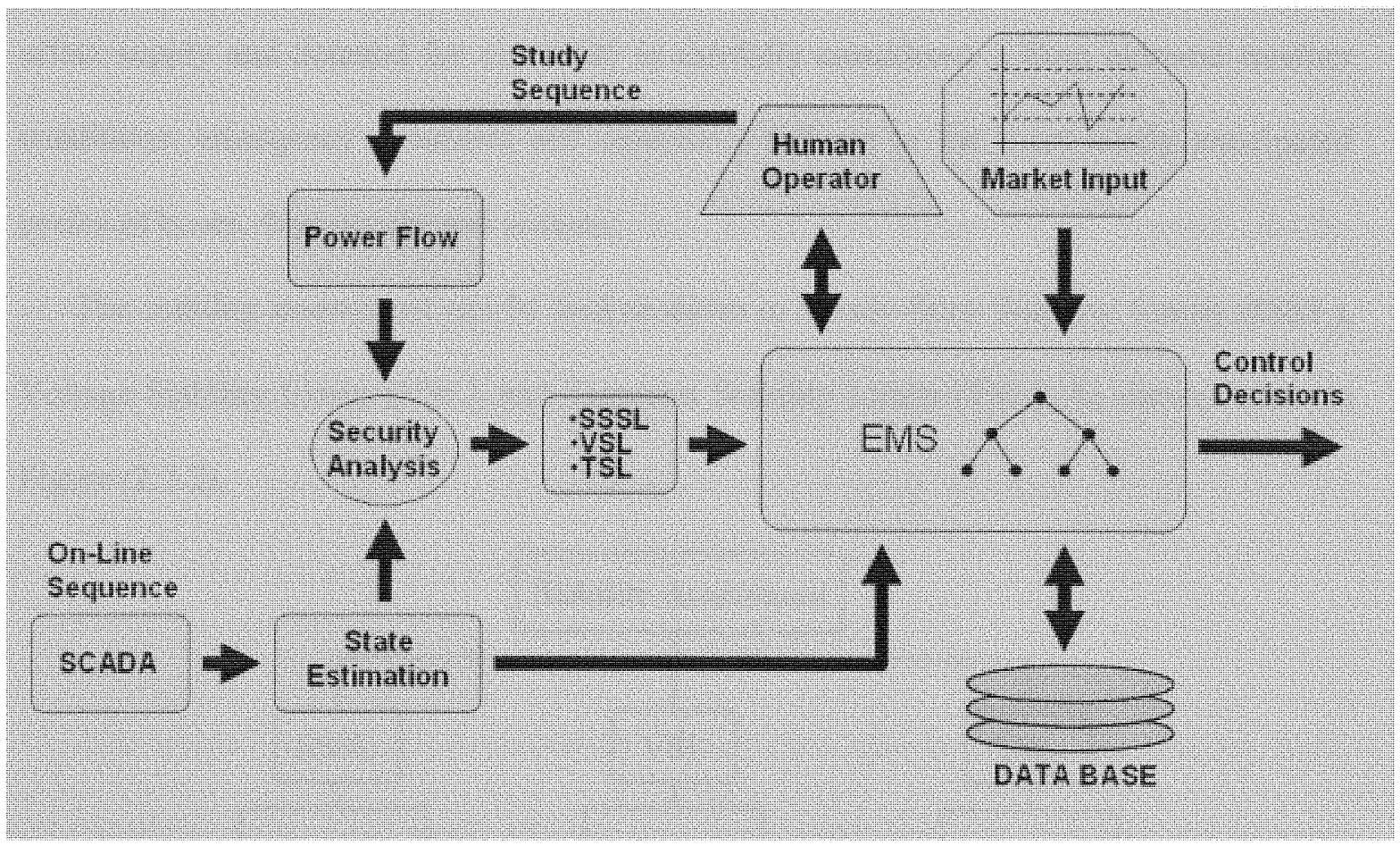

Figure (2.2.1) State-of-the art architecture of an EMS at the control center. 
Also, RTOs coordinate the continuous buying, selling and delivery of wholesale electricity through robust, open and competitive markets. In operating the markets, RTOs balance the needs of suppliers, wholesale customers and other market participants and continuously monitors market behavior. The wholesale electricity market is similar to a stock exchange. It establishes a market price for electricity by matching supply with demand. Online-tools make trading easy for members/customers by enabling them to submit bids and offers while providing them with continuous real-time data.

At the control center, power engineers and operators study thousands of "what if" scenarios and prepare to deal with virtually any event. Their power system security assessment consists of a set of procedures and methods used to determine whether the system is in a secure (stable) or in alert (insecure, unstable) state. Each variable that might affect supply and demand for electricity is carefully considered - from extreme weather conditions, emergency situations and equipment failures to the more easily anticipated cycles of hours, days, weeks and seasons.

The stability assessment can be broken down in Steady-State Stability Limits (SSSL), Voltage Stability Limits (VSL), and Transient Stability Limits (TSL). As shown below in Figure (2.2.2).

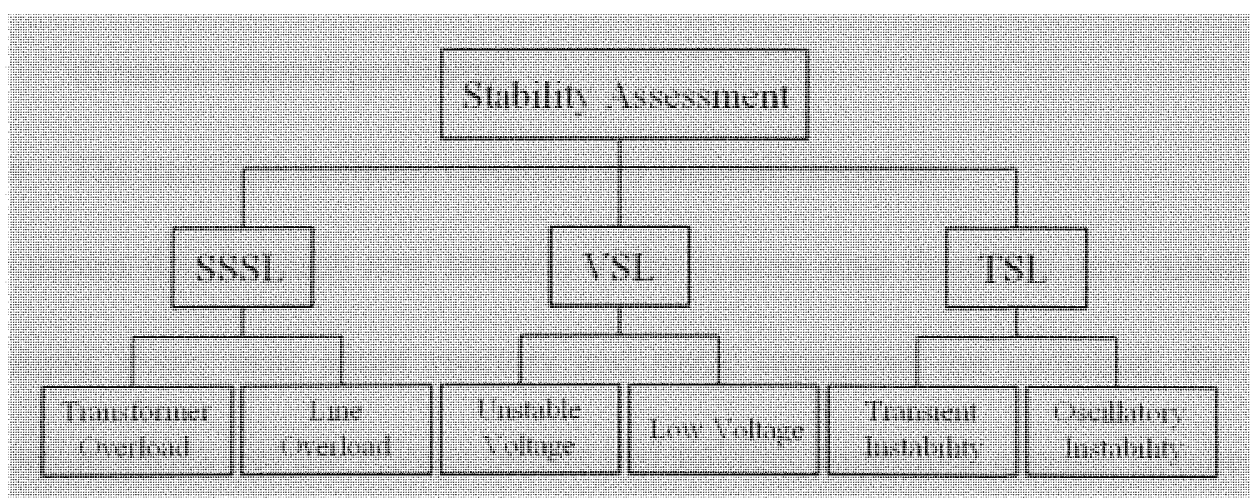

Figure (2.2.2) Security assessment for stability limits of the power system. 
Parameters such as voltage violations, frequency changes and thermal limits are easily identified using on-line assessments, and Available Transfer Capability (ATC), Total Transfer Capability (TTC), and Transmission Reliability Margin (TRM) are straightforward calculation, especially for local monitoring. Once the stability limits are set for the scheduled system's loading; the TTC and TRM can be easily calculated and locally monitored in real-time. However, the TSL is basically impossible to be determined in realtime. Therefore, TSL is determined off line, this is one of the main limitations of bulk control infrastructure. Further definitions and details can be found in Appendix A

Even with the centralized state-of-the art system described above, there is a wide range of power system decision problems, traditionally falling under one of the three categories of operation, maintenance, and planning.

Some of these decision problems include generation dispatching, fuel scheduling, control-room preventive and corrective actions, incident restoration, transmission service scheduling, unit commitment, transmission equipment maintenance, control system planning and transmission upgrade. All of them share common attributes, which are:

- The stability limit is a local property of the system state vector.

- Complex and computational intense application are required to solve the dynamic control problems.

- The essential decision variables are inherent uncertain.

This means that for each new solution of the system state there is a new stability limit. For example, every time shunt reactors and static capacitors are switched, the reactive compensation varies and the voltage profile and stability conditions change. In other words, stability limits exists and are not fixed and change with the system's loading, voltage and 
topology. This "changing nature" of the system stability is the main problem for on-line assessment because it requires very high computational speed to be able to compute stability limits and then utilize the results to perform corrective control actions.

Therefore, centralized infrastructures are not adequate to react effectively to local operating constrain violation or suppress it in time before causing a cascading failure.

\subsection{The Dynamic Control Problem (DCP)}

Dynamic control problems (DCP) can be described as problems whose variables represent the state and the control inputs to the system network. The network constrains are the representation of its dynamic and operating requirements [6].

Large networks such as electric power grid contain many devices that are constantly controlled to maintain the network under its security limits and maximize its operation. The adequate operations of such networks rely on the solution of DCP. In other words, the security of an electric power system depends on the ability of the control system to solve the DCP.

Whenever a contingency occurs, imbalances between these quantities force some generators to speed up while others slow down. If the difference in angle between any two machines increases indefinitely or if the oscillatory transient is not sufficiently damped, the system will become unstable; the generators will lose synchronization and, consequently, the system will collapse [11]. The core of the problem is the fact that mechanical adjustments are much slower than electric changes.

For example in electric power systems, generators can be modeled by two components; a mechanical part (turbine) and an electric part (generator). 
The mechanical power input and electric power output are identical in steady-state stable condition as in equation (2-1).

$$
\mathrm{T}_{\mathrm{a}}=\mathrm{T}_{\mathrm{m}}-\mathrm{T}_{\mathrm{e}} \quad \mathrm{N} \cdot \mathrm{m}
$$

Where, $T_{a}$ is the accelerating torque in Newton-meters. $T_{m}$ is the mechanical driving torque and $T_{e}$ is the electrical or load torque.

The dynamic equation or swing equation that governs the motion of the machine rotor relating the inertia torque to the resultant of the mechanical and electrical torques on the rotor are described in (2-2) and (2-3).

$$
\begin{gathered}
\dot{\delta} \propto \omega \\
\omega=\frac{\omega_{0}}{H} \mathrm{Pm}-\frac{\mathrm{D}}{\mathrm{H}} \omega-\frac{\omega_{\mathrm{o}}}{\mathrm{H}} \mathrm{Pe} \mathrm{rad} / \mathrm{s}^{2}
\end{gathered}
$$

Where, $\delta$ is the related rotor torque angle of the generator, $\omega$ is the rotor speed deviation of the generator after any change, $\omega_{0}$ is the initial speed of the generator. $H$ is the effective inertia of the generator, $\mathrm{D}$ is the damping factor of the generator and Pe is the electric power injection from the generator to the network.

This thesis applies distributed real-time control solution approach to address the DCP in power system networks. To investigate the potential of distributed control, the thesis presents experiments in a representative power networks model, in which the control task is to restore (after major disturbances) the synchronous mode of the generators. The system setup and results for the experiments are detailed in the later chapters. 


\section{Chapter 3}

\section{Distributed Control Infrastructure}

This chapter covers the basics of Distributed Control Infrastructure, its advantages and its potential implementation in the electric networks [5].

The opportunity of implementing distributed control in electric networks lies in the potential of improving performance, adaptability, robustness, and overall security of the grid. The research efforts in this thesis consist of applying the principles of distributed control infrastructures in an electrical system to evaluate its advantages over central control infrastructure.

\subsection{Distributed Control and Software Agents}

A distributed control system can be described as a control system with distributed processor capacity. Distributed control infrastructures for power systems utilize software control agents assigned to specific controlled equipments to perform specific set of tasks.

This software-control agent solves its local problem, and in coordination with neighboring agents to achieve the global system objectives in an optimal way. The local control action and coordination between agents happens in two different levels, the agent will periodically update its status through interaction at the second level.

The $\mathrm{N}$ distributed subsystems, of the large-scale power system, generates local solutions at the $\mathrm{N}$ subsystems, and then provides a new set of control parameters for the entire power system.

The different control decisions generated at the second level are then arranged by the system coordinator to provide a feasible solution to the global problem see Figure (3.1.1). 
The coordinator's objective is to avoid too strong corrective control actions originated from the situation where the control force is stronger than the system's oscillatory force.

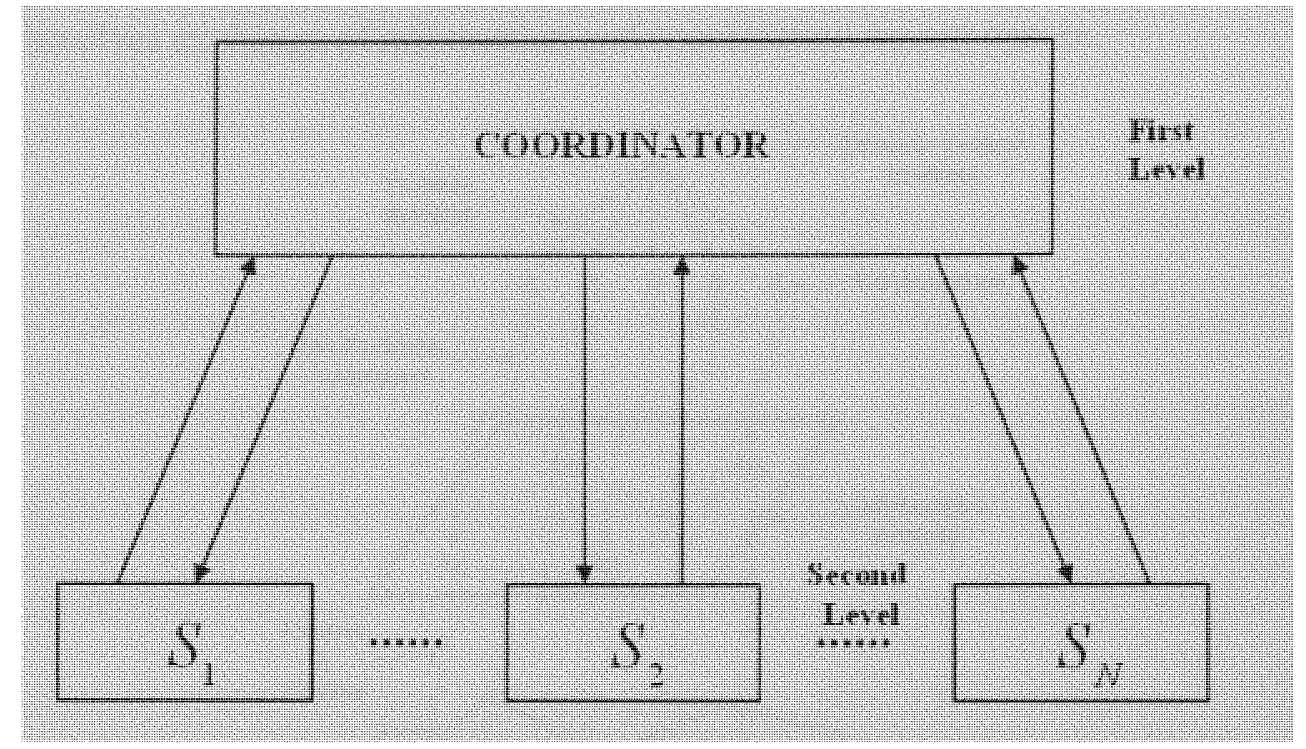

Figure (3.1.1) Multilevel distributed control system.

This coordination scheme requires a collaborative network to exchange information between neighboring agents and accomplish the common goal.

Unlike centered control infrastructures, the distributed control infrastructure can provide faster response to quickly solve the system's Dynamic Control Problem (DCP) and therefore increase the reliability margin of the system. The reliability margin accounts for the uncertainties associated with the transmission system where the power transfer operating point should be below the reliability margin.

\subsection{Distributed Control Infrastructure Solution for DCP}

Figure (3.2.1) shows an illustration of the solution approach for DCP based on a distributed control infrastructure. Note that the dynamic control problem (P), breaks up into a set of small and localized sub-problems, $\left\{\left(P_{1} \ldots P_{m}\right)\right\}$, then matches and engages the software-agents $\left\{\left(\mathrm{Ag}_{1} \ldots \mathrm{Ag}_{\mathrm{m}}\right)\right\}$ in solving the sub-problems $\left\{\left(\mathrm{P}_{1} \ldots \mathrm{P}_{\mathrm{m}}\right)\right\}$. 
These agents are independent, and set in a collaborative network. Independence refers to the agent's ability to make decision without central supervision. The collaborative network involves exchange of information and coordination among agents.

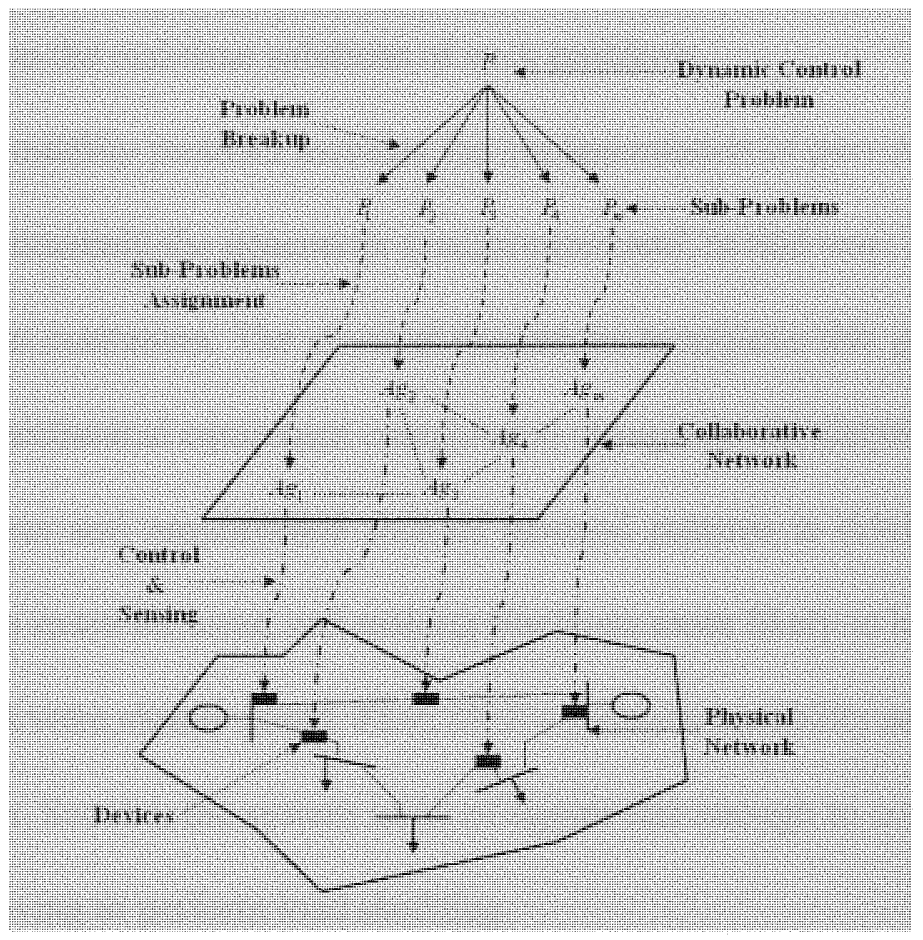

Figure (3.2.1) An illustration of the distributed control solution for DCP.

Because of the size of the physical network, the agents only share information and have coordination with a near neighbor. Even though in the case of power system network there is only one layer for decision making, the coordination level is embedded in the collaboration protocol for this infrastructure.

\subsection{Distributed Control Infrastructure in Power System Networks}

This section concentrates on the transient stability problems of the power system, the limitations of its actual control system, and how distributed control would address these problems enhancing the speed of response of the control system. 
As previously stated, the standard solution for DCP in power system is based on offline system assessment done by a centralized optimizer computer and SCADA systems. Such inflexible system is not adequate to address DCP given that the secure operation of the system relies on the real-time solutions of such problems.

The transient stability problem includes the implementation of preventive and real-time control actions to maintain the generators synchronized and, in that way, sustain continuous supply of power to customers. The physical limitations to achieve this goal lie in the slow reaction time of mechanical equipment compared to changes in the electric equipment.

The continuous and unscheduled shift of demand patterns is driving the grid to its limits, leaving less space for error, and making blackouts more likely to happen.

Two problems were clearly stated. First, DCP requires adaptability and fast response of the control system. Second, the system lacks of agents fast enough to control the electrical system without having to perform load shedding.

New breakthrough technologies such as solid-state power control devices or Flexible AC Transmission Systems (FACTS) are being developed to provide a quick response to system disturbances. These devices can be switched at high speed so as to influence electric-power output and, thereby, compensate for its imbalance with mechanical-power input, absorb oscillations, and preserve the synchronization of the generators.

To demonstrate the effectiveness of the distributed control solutions on DCP (transient stability), we developed a distributed control infrastructure that utilize FACTS devices as distributed agents throughout the power system.

Details on the telecommunication network infrastructure implementation are discussed in the following chapter. 


\section{Chapter 4}

\section{Telecommunication Network Infrastructure}

As stated before, agents are independent but set in a collaborative network. This collaborative network implies data sharing for control coordination. Due to the importance of collaboration and coordination, a rigid telecommunication network is required, between agents in order to share data, and control signal. The structure of the collaborative network and the telecommunication network is outlined in this chapter.

\subsection{Distributed Control Infrastructure and Telecommunication Network}

Synchronous networks (SCADA and EMS systems) are coordinated under a centralized control and developed to solve hard off-line optimization problems, whereas collaborative networks are being developed to solve online optimization and local control problems.

Collaborative networks focuses on the structure, behavior, and evolving dynamics of networks of autonomous entities that collaborate to better achieve common or compatible goals. Collaborative networks, together with distributed agents and problem separation, form the core solution to solve dynamic control problems in a distributed control infrastructure.

The distributed agent's model has two sub-systems, the control sub-system (agent) and the equipment sub-system (controlled device) as shown in Figure (4.1.1). The agent's collaborative network requires a telecommunication network infrastructure to share data and to coordinate control action among them. The proposed telecommunication network incorporates industry standards Internet Protocol (IP) protocol for the agents at network layer, and Transport Control Protocol (TCP) at the transport layer. 


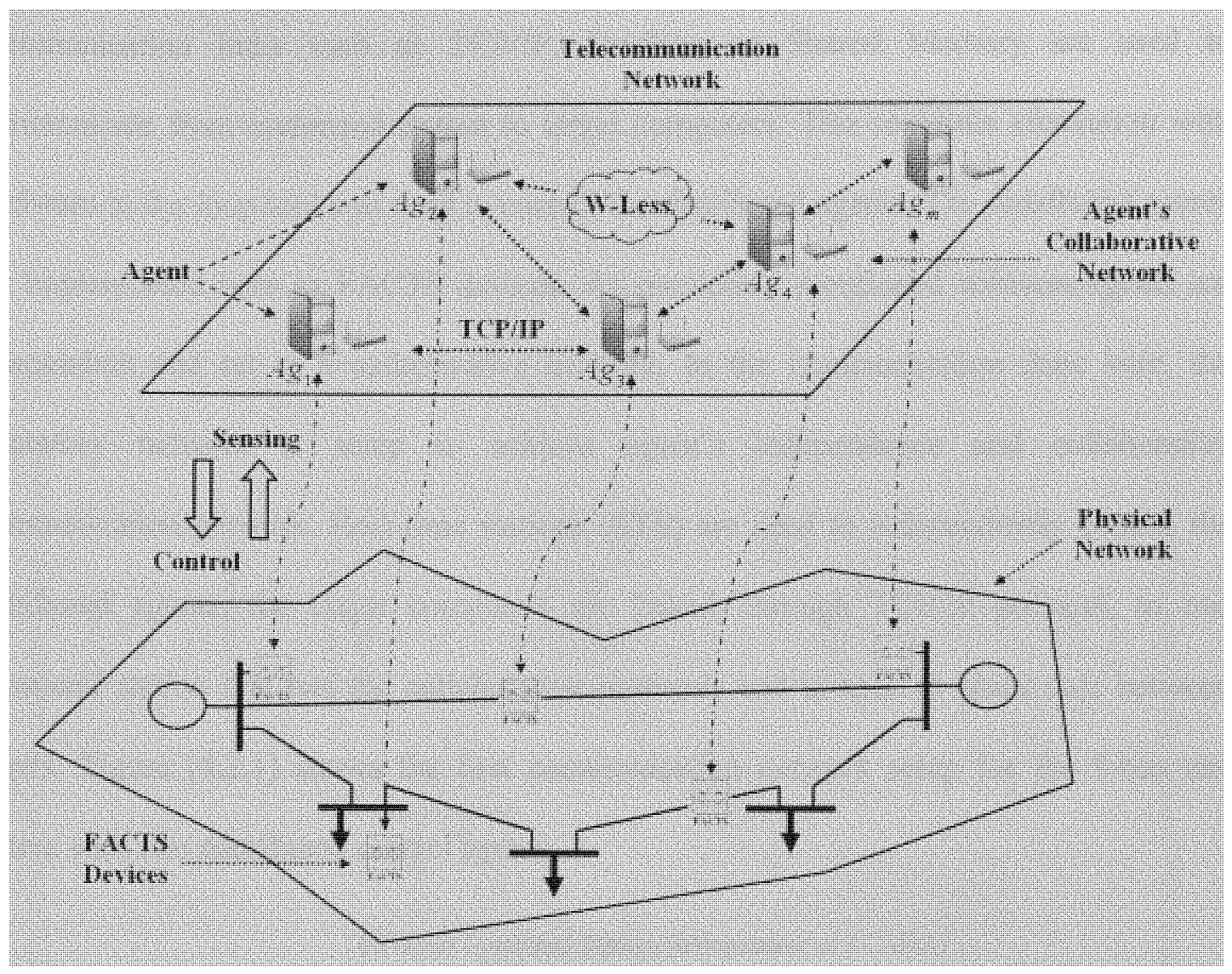

Figure (4.1.1) Collaborative and telecommunication network.

The agents programs are matched to specific FACTS control system in the physical network, also they communicate with the nearest neighboring agent to share local values and control schemes.

\subsection{Telecommunication Network Methods of Implementation}

Two different layers of communication networks were implemented into the power system simulator developed here.

- The collaboration network for the distributed control infrastructure.

- The SCADA environment for the central control infrastructure.

First, the implementation of real-time control with hardware in the loop requires the simulation to be distributed over several processors to achieve real-time operation. 
Communication links such as TCP/IP, FireWire or Shared Memory are used to communicate from command station to target computers. This real-time simulation with hardware in the loop tool was used to control the distributed agents. The distributed agents are implemented here as FACTS devices. As previously mentioned software-agents are independent and set in a collaborative network. In the implementation done here, the laboratory network is setup to achieve agent's independence and collaborative communication network. Real-time stations (RTS) are used to independently control the distributed agents; the target computers are linked, through TCP/IP protocol, to one command station that has the collaborative protocols and is used to share data between targets. Figure (4.2.1) shows the first communication network setup for the 9-bus test system with two distributed control agents.

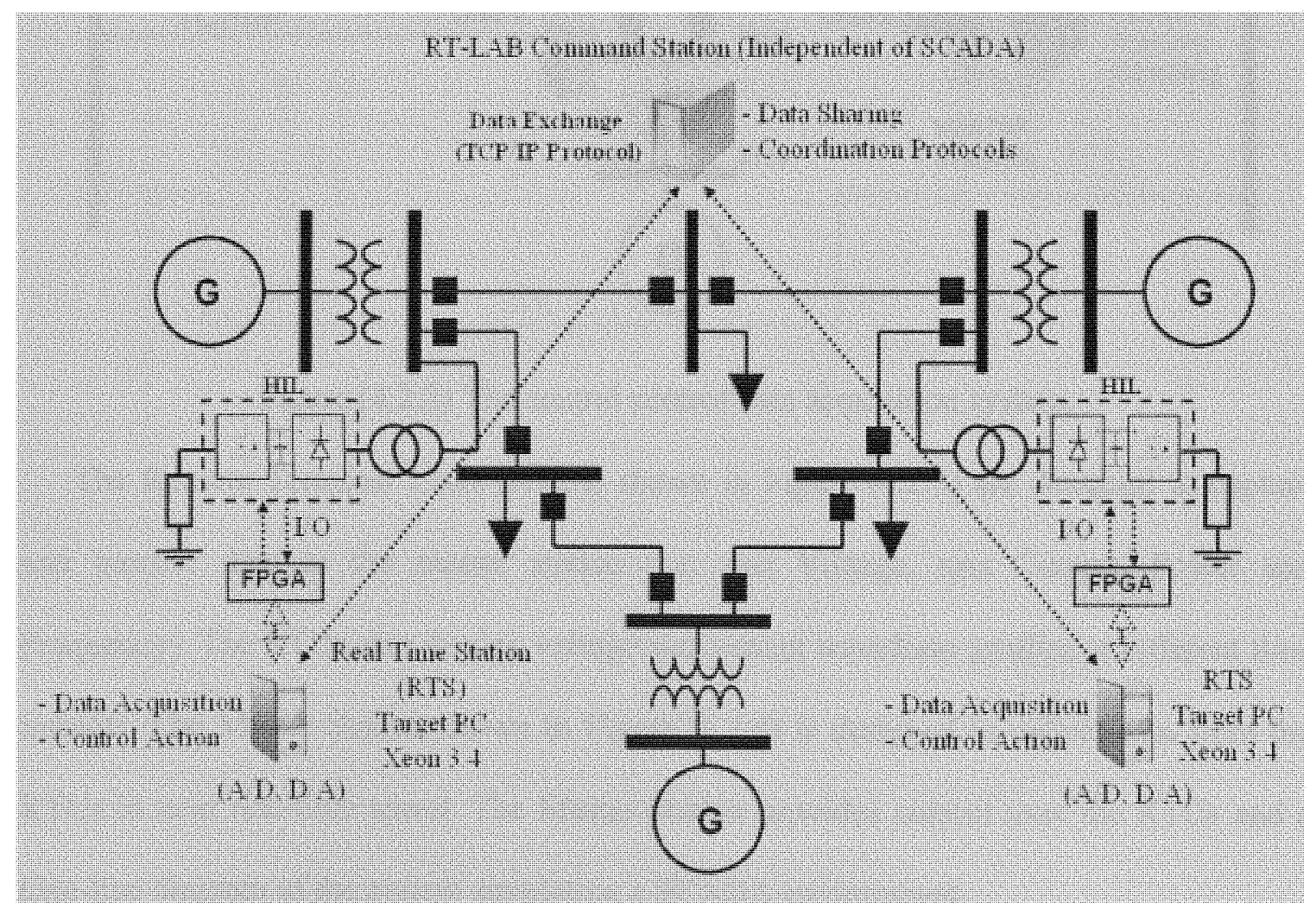

Figure (4.2.1) The communication setup for the 9-bus system with two FACTS devices. 
Both agents with its respective FACTS devices are located as near as possible to the generators for a more efficient operation. The agents and its controlled FACTS devices are interfaced through a FPGA card for data acquisition and control action.

The communication layer for the SCADA system is independent of the first one. Figure (4.2.2) shows the central control data acquisition system.

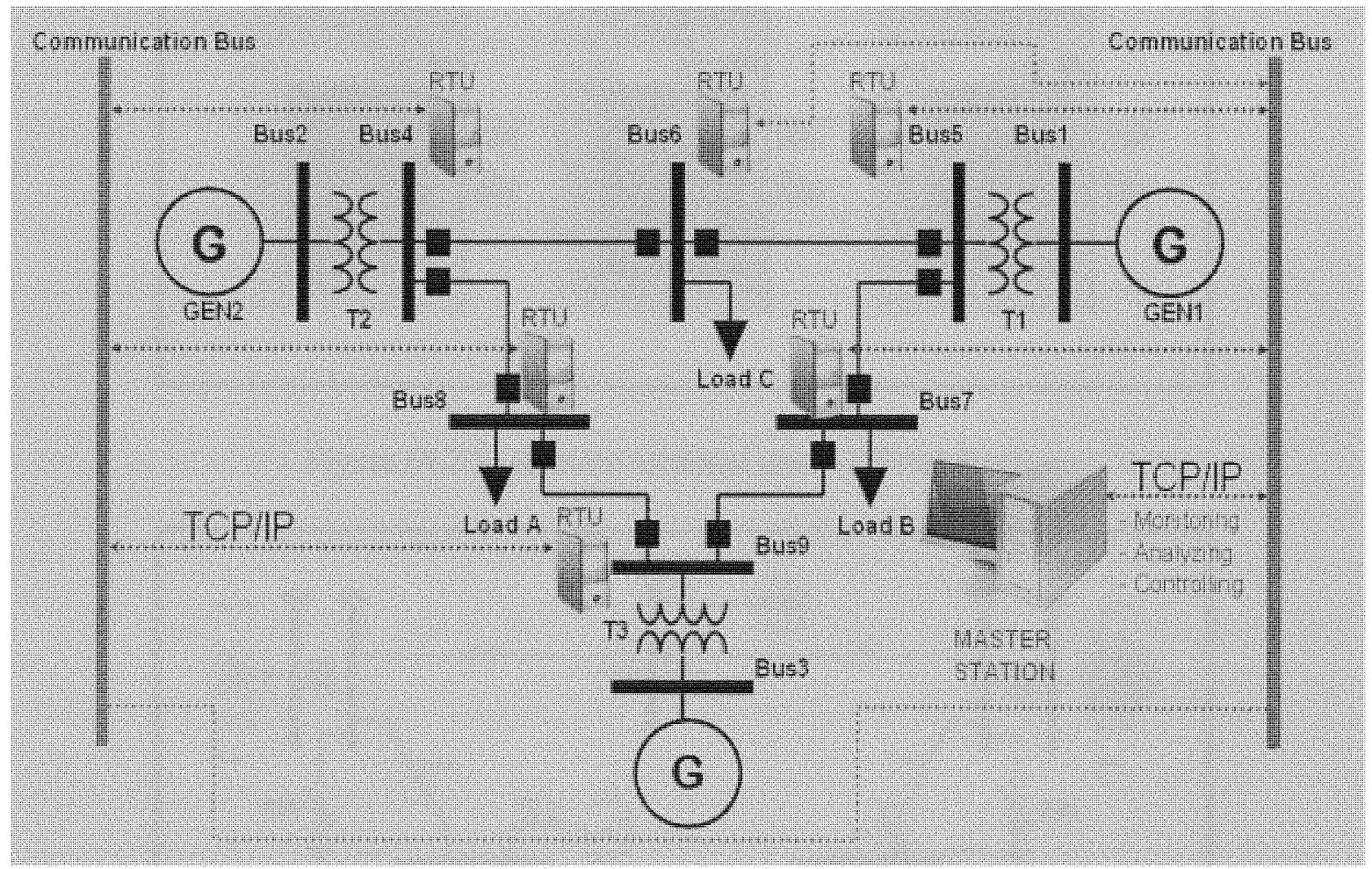

Figure (4.2.2) The central control data acquisition system.

The Remote Terminal Units (RTUs) shown in Figure (4.2.3) are designed to capture and transfer data to the master station (i.e. rms Voltage, rms Current, Power and Frequency). The RTUs also display voltage and current waveform profiles at different time instants. The Master-Station shown in Figure (4.2.4) is designed to collect data from the RTUs in near real-time speed. The Master-Station provides control over the RTUs and a One-line diagram of the system simulating a SCADA environment. 


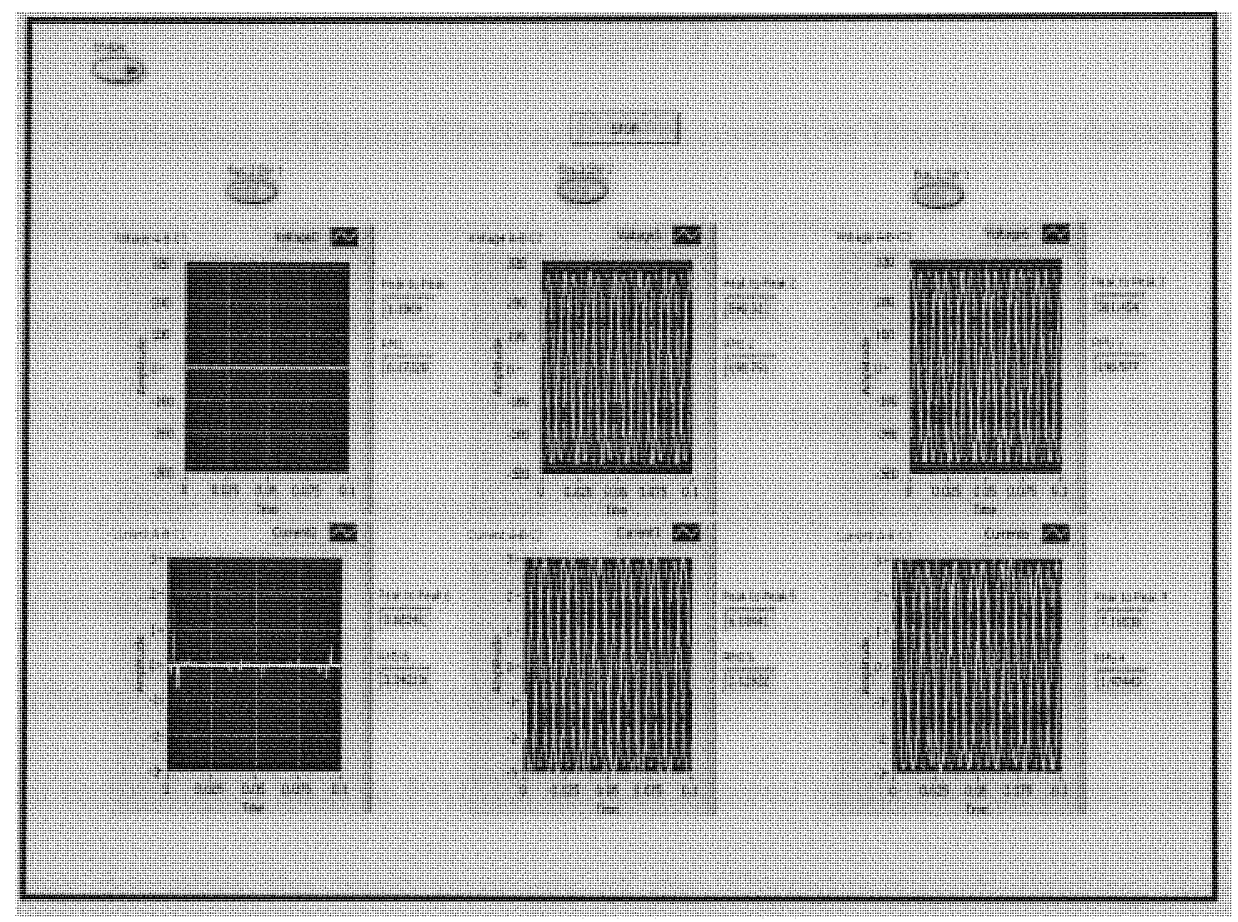

Figure (4.2.3) Remote terminal units (RTUs) display.

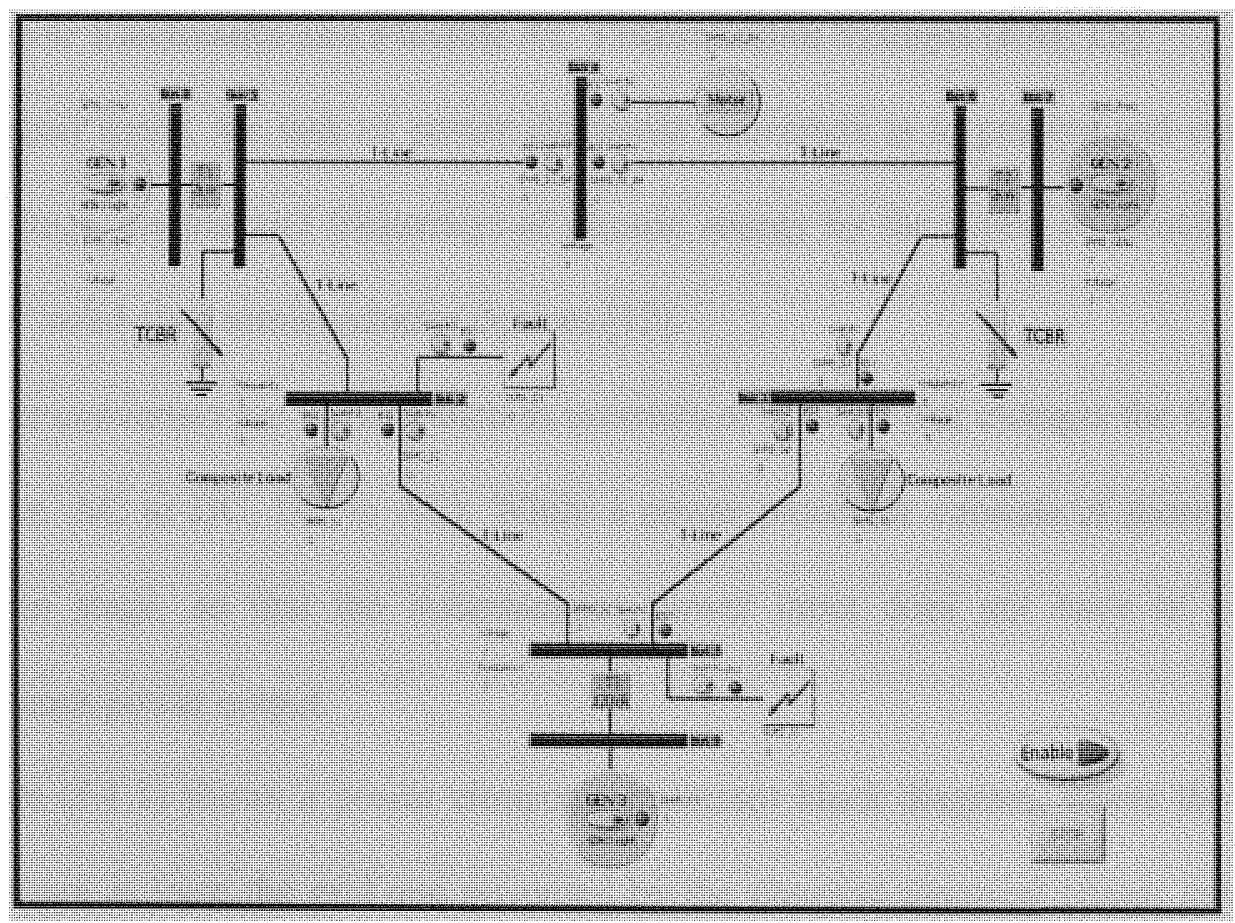

Figure (4.2.4) Master station display. 


\section{Chapter 5}

\section{Application of Power Electronics Processors as Distributed Agents}

The discussion of distributed control given earlier conveyed the idea that the distributed control approach encompasses collaborative networks, problem division and distributed agents. It was also mentioned that there is a lack of agents that can act fast enough to control the power system oscillations without having to perform load shedding or triggering protection relays.

This chapter outlines the opportunities, advantages and capabilities of power electronic processors technologies such as FACTS devices to serve as distributed agents. Specifically, this chapter describes the basic concepts of the Thyristor-Switch Braking Resistor, its application on transient stability problems and its potential as a distributed control agent.

\subsection{Power Electronics and Flexible AC Transmission Systems (FACTS)}

In broad terms, the task of power electronic converters is to process and control the flow (voltage, current and frequency) of electric energy by the action of switching "On" and "Off" solid-state semiconductor devices [12].

Power converters are devices such as inverters or rectifier that changes the form of the power (i.e. $\mathrm{AC}$ to $\mathrm{DC}, \mathrm{DC}$ to $\mathrm{AC}$ ). While a power processor consists of more than one conversion stage, so that the instantaneous power input does not have to equal the instantaneous power output.

Figure (5.1.1) shows a power electronic processor block diagram, where the input to the processor is from the grid and governed by the physical-electrical circuit laws, while the output of the processor is a controllable factor (i.e. voltage, current, frequency) that can be optimally suited for a specific purpose. 


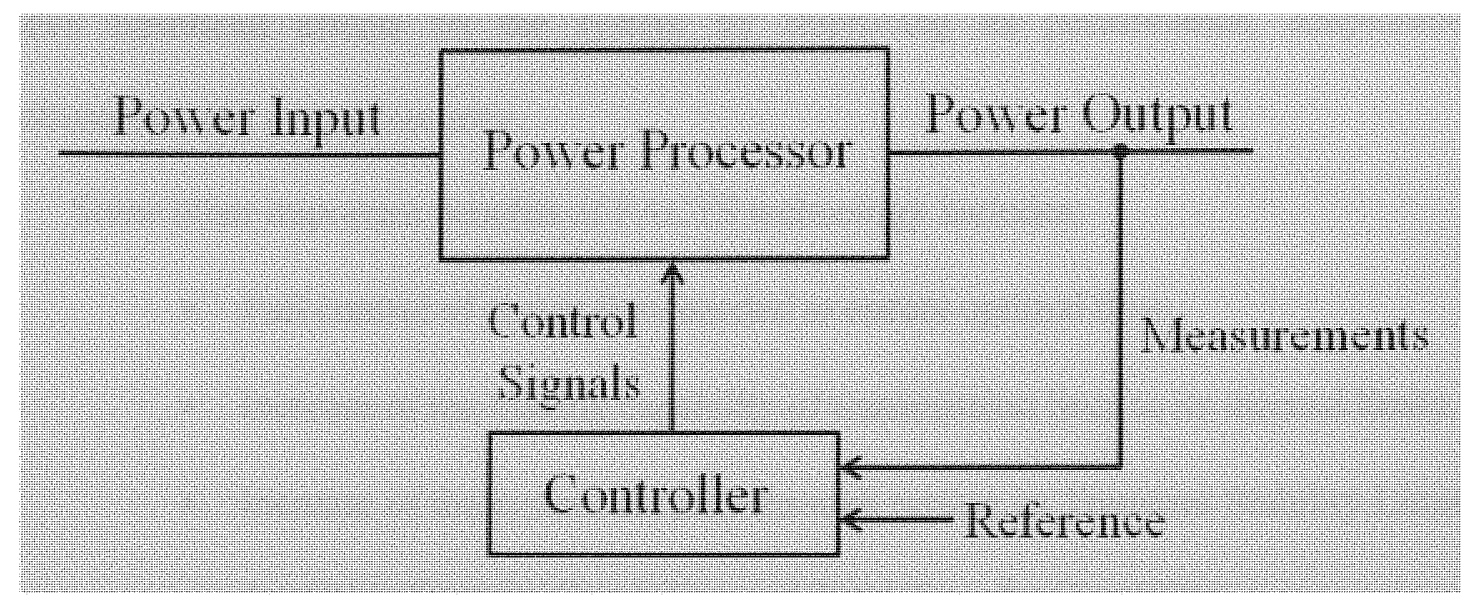

Figure (5.1.1) Block diagram of a power electronic processor.

Flexible AC Transmission Systems include one type of power processor that is revolutionizing all areas of electric energy. FACTS involves conversion and/or switching power electronics in the range of a few tens to hundreds megawatts, allowing fast and direct control on some parameters of power systems, such as line and shunt impedances.

\subsection{Application of FACTS Devices as Distributed Agents}

FACTS devices open new opportunities for controlling power and enhancing the overall security and reliability of power systems. This is possible due to the ability of FACTS devices to control interrelated parameters that governs the operation of the system including series impedance, shunt impedance, current, voltage, phase angle, and damping of oscillation at various frequencies. All this constraints can not be overcome by mechanical action or switching because of its characteristically slow response and discontinuous control action [13].

Full control of the system's parameters combined with fast response makes FACTS devices the best candidate to perform as an autonomous agent. The use of FACTS devices as distributed agents together with the distributed control and coordination algorithms will 
have beneficial effects on the system by improving its performance, adaptability, robustness, and overall security.

Some other important benefits of FACTS technology includes:

- Full control of power flow needed to ensure optimal flow.

- Increase of the loading capability of transmission lines to its thermal limits.

- Increase of the system security through raising its Transient Stability Limits, Available Transfer Capability, and Total Transfer Capability.

- Provides secure tie line connections to neighboring utilities.

- Provides flexibility in sitting new generation.

- Reduces reactive flow enhancing real power flow.

\subsection{Thyristor Switch Braking Resistor (TSBR)}

To demonstrate the effectiveness of the distributed control approach for dynamic control problems, we developed a distributed control strategy that employs Thyristor Switch Braking Resistor (TSBR) FACTS-type devices as distributed agents. This section describes the basic concepts of TSBR, its design and operation.

The TSBR in Figure (5.3.1) is a shunt-connected Thyristor-switch resistor. This TSBR, also referred as dynamic brake, is controlled on and off to aid in the stabilization of power system transients and sub-synchronous oscillations by reducing the net available energy for acceleration and hence the speed deviation of the generating unit during a disturbance.

The best location for a TSBR is near a generator that would need braking during transient stability conditions. Given the appropriate rating and control, a TSBR can be designed to simultaneously undertake multiple functions. The three phases can be connected in delta or wye, although delta would be more convenient because it does not 
need a ground path (See Figure (5.3.2). The TSBR is a simple, cost effective and highly reliable FACTS controller.

TSBR can be utilized for:

- Preventing instability during the first power swing cycle.

- Damping sub-synchronous resonance resulting from capacitor compensation.

- Enhancing damping and preventing dynamic instability involving low frequency oscillations.

- Aiding the synchronization of a generator to the grid.

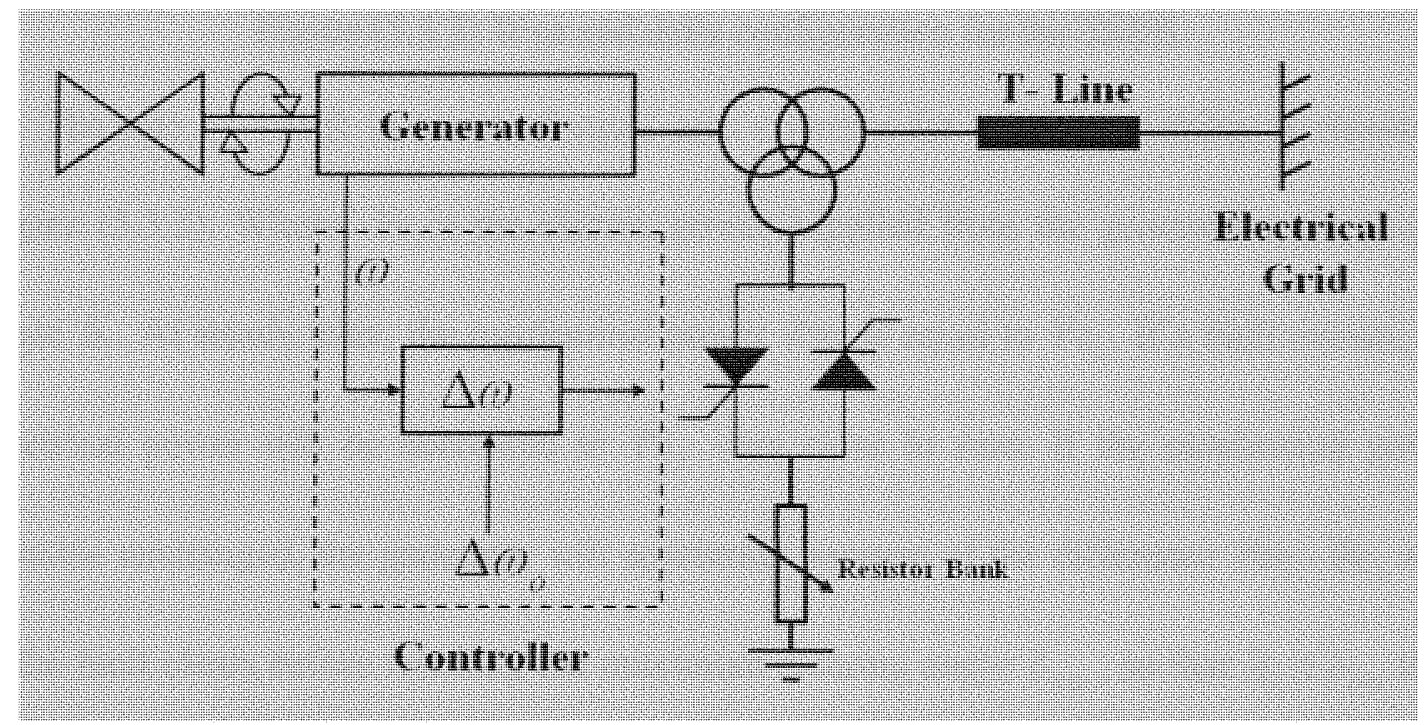

Figure (5.3.1) One-line diagram including TSBR.

This FACTS device could be optimized to damp the sub-transient oscillations by adding an energy storage device such as a superconducting Magnetic or battery system, or even a dc capacitor-based storage [13]. This will allow the FACTS to operate not only in the braking mode but also in the energy supply mode. 


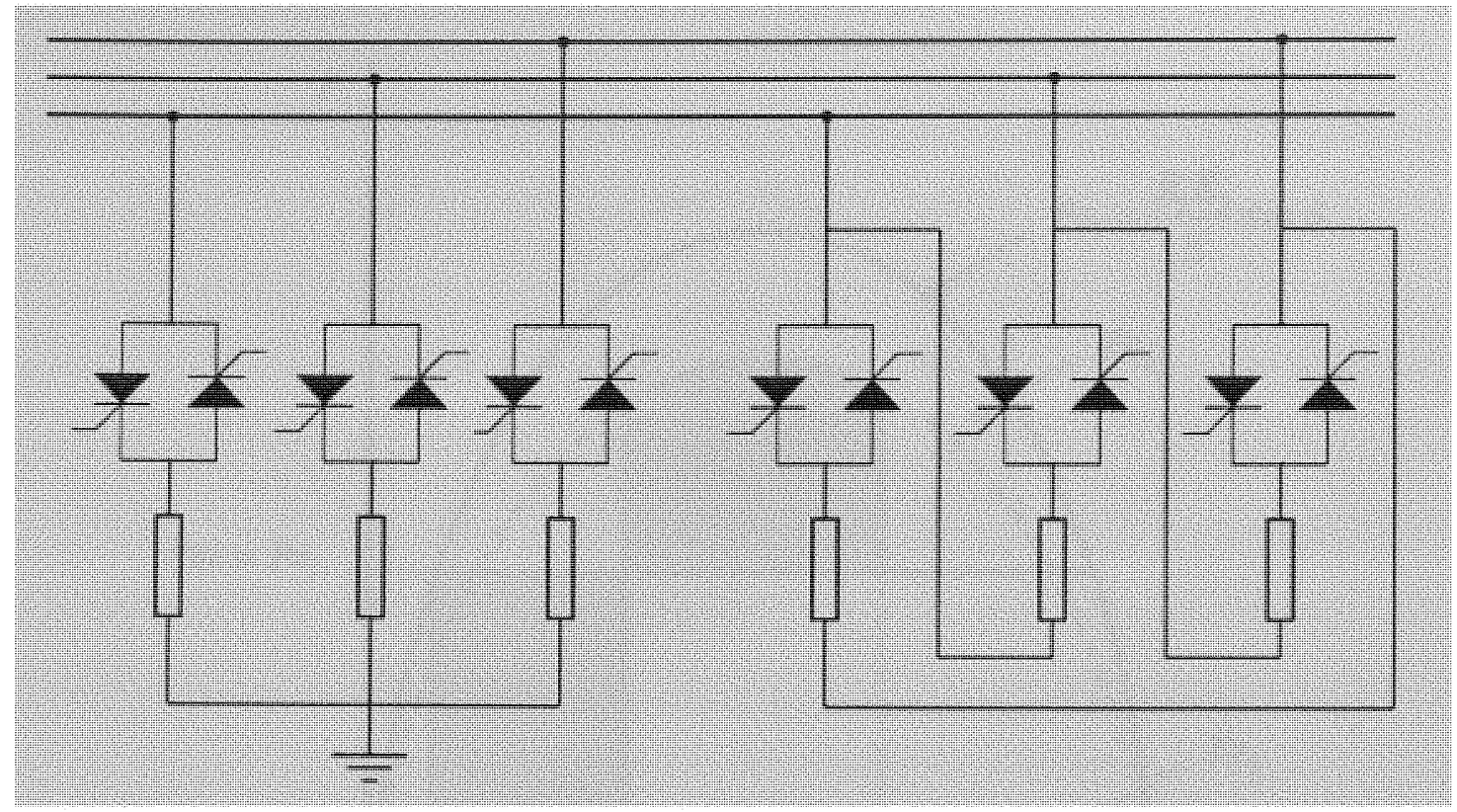

Figure (5.3.2) Wye and delta configuration for the TSBR.

The role of TSBR in terms of equal area criterion for transient stability is shown in Figure (5.3.3). Where, $\mathrm{Pe}_{1}$ is the electric power supplied from the generator with a rotor angle $\delta_{1}$. At steady state, the mechanical power $\mathrm{Pm}$ is equal to $\mathrm{Pe}_{1}$. If a fault occurs, the electrical power will be decreased to $\mathrm{Pe}_{2}$ very close to zero. If the mechanical power remains unchanged, the generator will accelerate because of the difference $\mathrm{Pm}-\mathrm{Pe}_{2} \neq 0$. If the fault clears before reaching the maximum clearing angle $\delta_{\max }$, so that $\delta_{2} \leq \delta_{\max }$ then the generator will remain in synchronism and reach a new steady state $\mathrm{Pe}_{3}$ at $\delta_{3}$ for the new values of $\mathrm{E}_{1} \mathrm{E}_{2} / \mathrm{X}$, where $\mathrm{Pe}_{2}<\mathrm{Pe}_{3}<\mathrm{Pe}_{1}$. Now, if the fault is cleared after reaching the maximum clearing angle $\delta_{\max }$, so that $\delta_{\max }<\delta_{2}$, then the generator will keep accelerating until it losses synchronism [14]. 


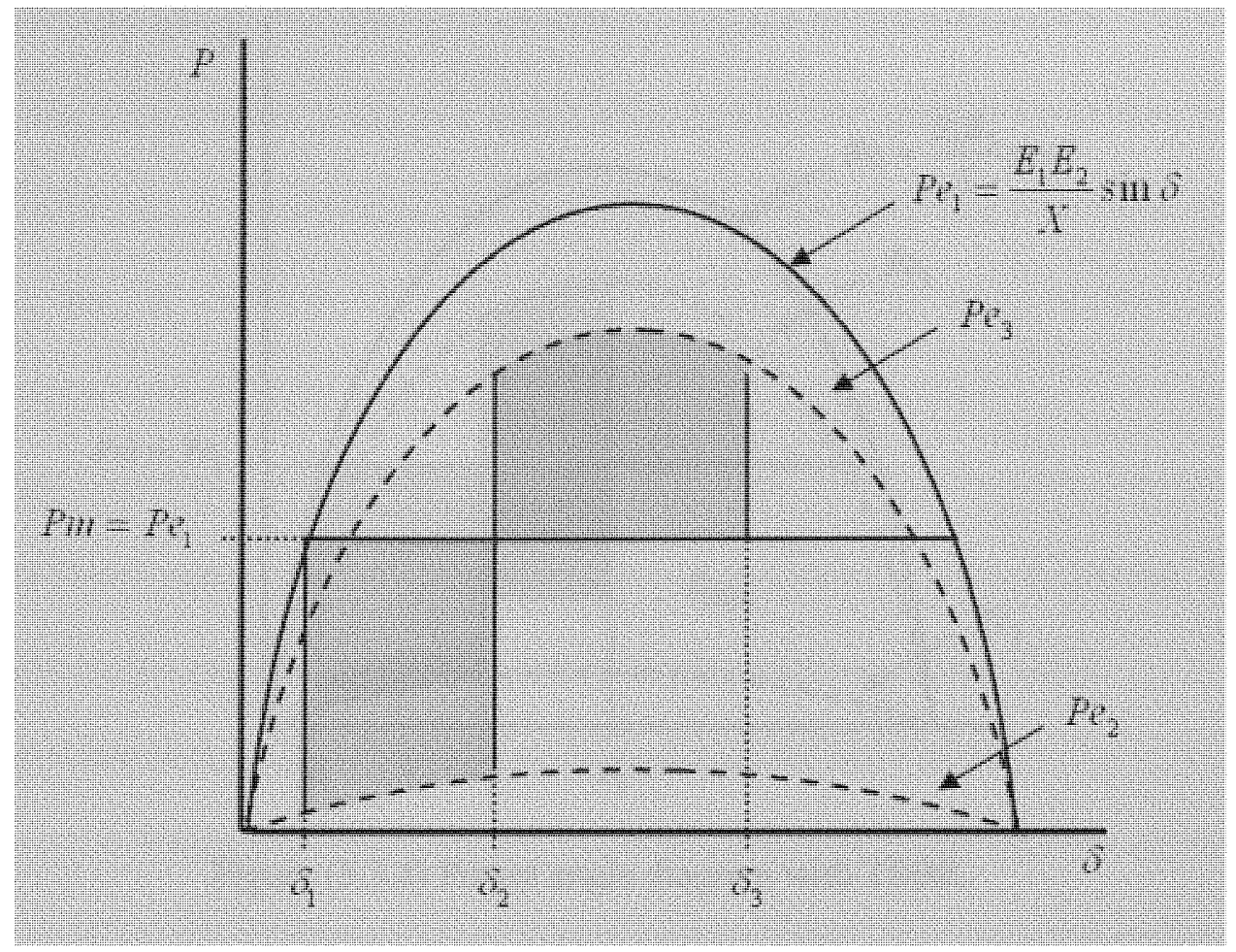

Figure (5.3.3) Power angle diagram without TSBR.

The TSBR can aid the transient stability by increasing the TSL and reduce the acceleration. Figure (5.3.4) shows the performance of the TSBR during the fault. The maximum angle $\delta_{3}$ is much smaller than in Figure (5.3.3), which denotes, that the TSBR increases the stability margin.

Figure (5.3.5) shows the waveforms in time domain for the swing, including the power angle, the speed deviation and the TSBR power. The control parameter for the TSBR is the speed deviation $\Delta \omega=\omega-\omega_{0}$, where $\omega$ is the actual speed of the generator and $\omega_{0}$ is the steady state speed for the generator or initial speed, is the control parameter for the TSBR. The TSBR is switched on when the speed deviation is positive and switched off when the speed deviation is negative. 


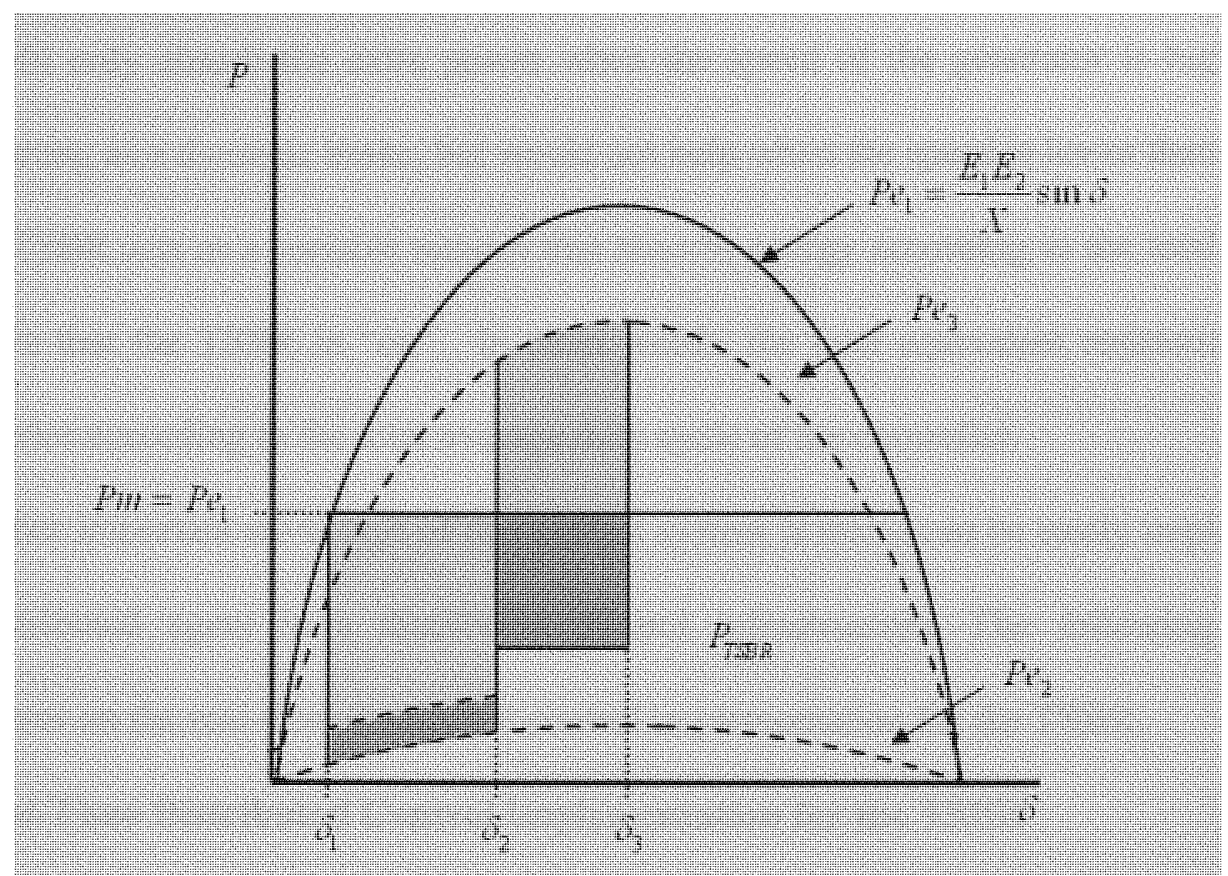

Figure (5.3.4) Power angle diagram with TSBR.

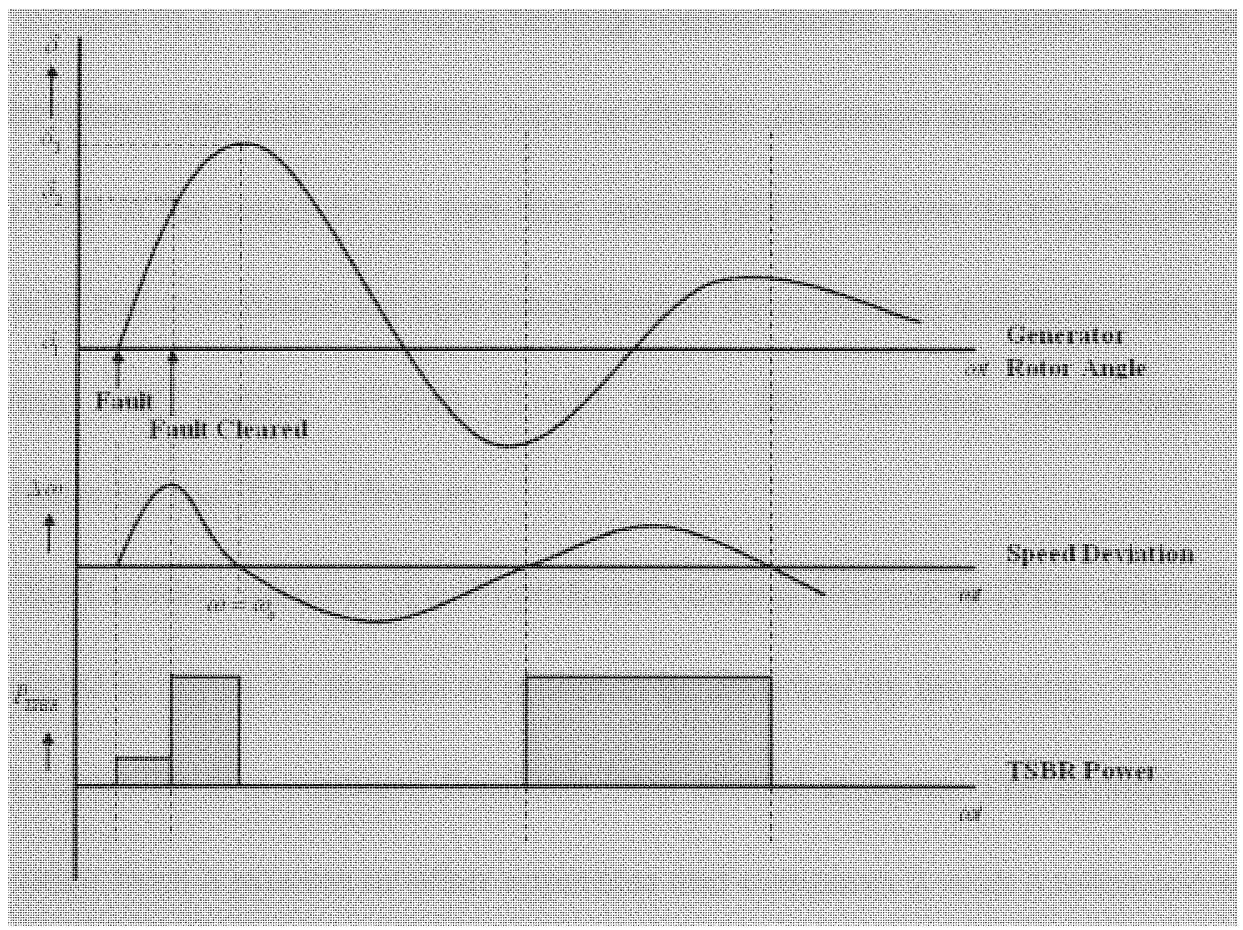

Figure (5.3.5) Operation for damping oscillation and dynamic instability.

During the fault the TSBR power will be zero or very small, and once the fault is cleared, the generator speed will increase and so will the power absorbed by the TSBR. The 
TSRB will only absorb the extra power when the speed of the generator is higher than normal acting as a dynamic brake, but would not help when the speed is lower than normal.

The TSBR acts as a variable resistor that absorbs energy according to the total average duty cycle that the TSBR in "on". In other words, the total equivalent resistance of the TSBR varies according to the total average duty cycle of the controller.

Another important advantage of this control system is that it can be easily implemented as a distributed agent in a distributed control infrastructure. The following section concentrates on the real-time distributed control implementation for the TSBR.

\subsection{Real-Time Distributed Control Implementation for the TSBR}

Figure (5.4.1) shows the main control block diagram which was used for closed-loop frequency control of a Thyristor switch braking resistor model. The control model consists of two subsystems; the first subsystem runs on the PC command station while the second subsystem runs at the target PC or real-time station (RTS), referrer to Figure (4.2.1). The control results and parameters are then transferred to the command station for data logging, data sharing and display. Figure (5.4.2) show the real-time distributed control implementation for two TSBR devices.

The TSBR control consists of a Proportional Integral Derivative (PID) controller with gains calculated to adjust the amount of power to be absorbed by the device.

A PID controller is a common feedback loop control systems. The controller takes a measured value from a process or other apparatus and compares it with a reference setpoint value. The difference is then used to adjust some input to process in order to bring the process' measured value to its desired setpoint. 


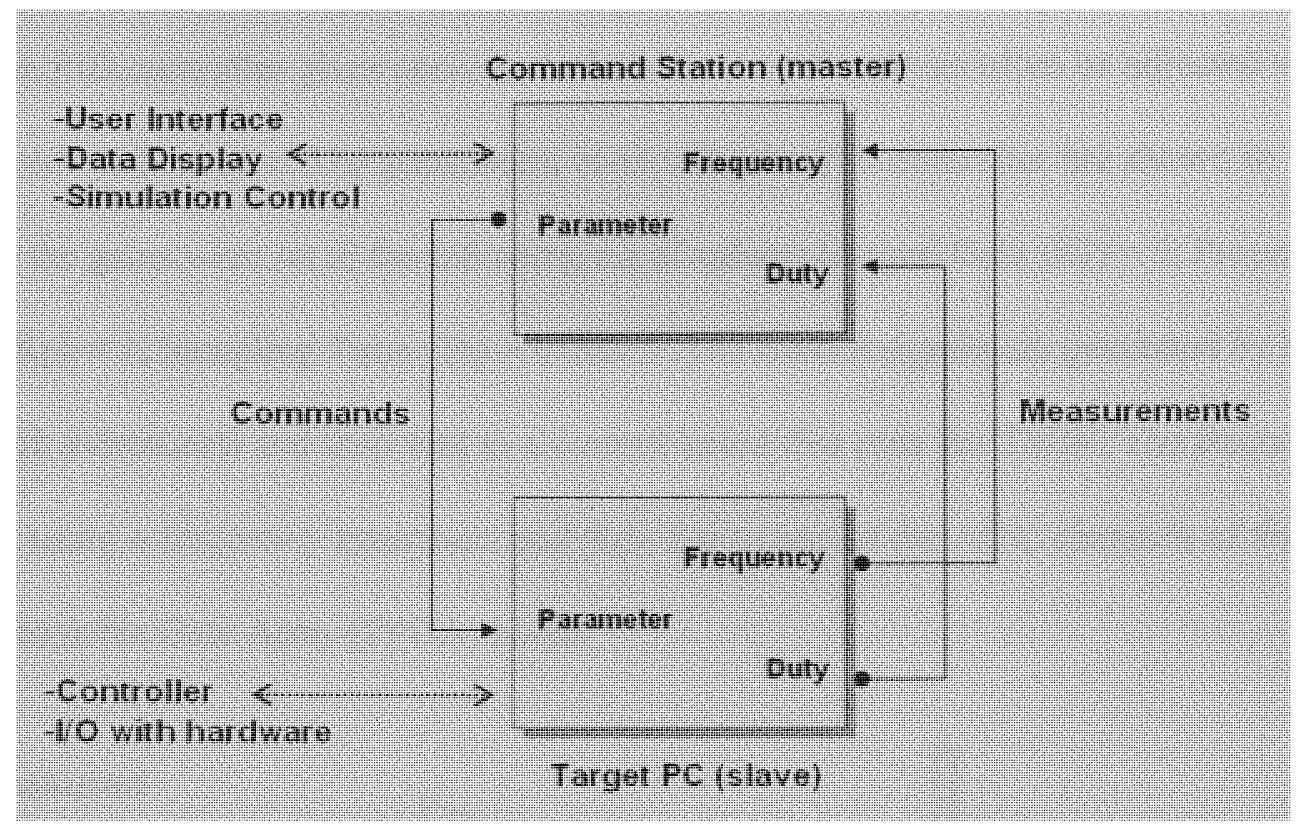

Figure (5.4.1) Real-time control block diagram for the TSBR.

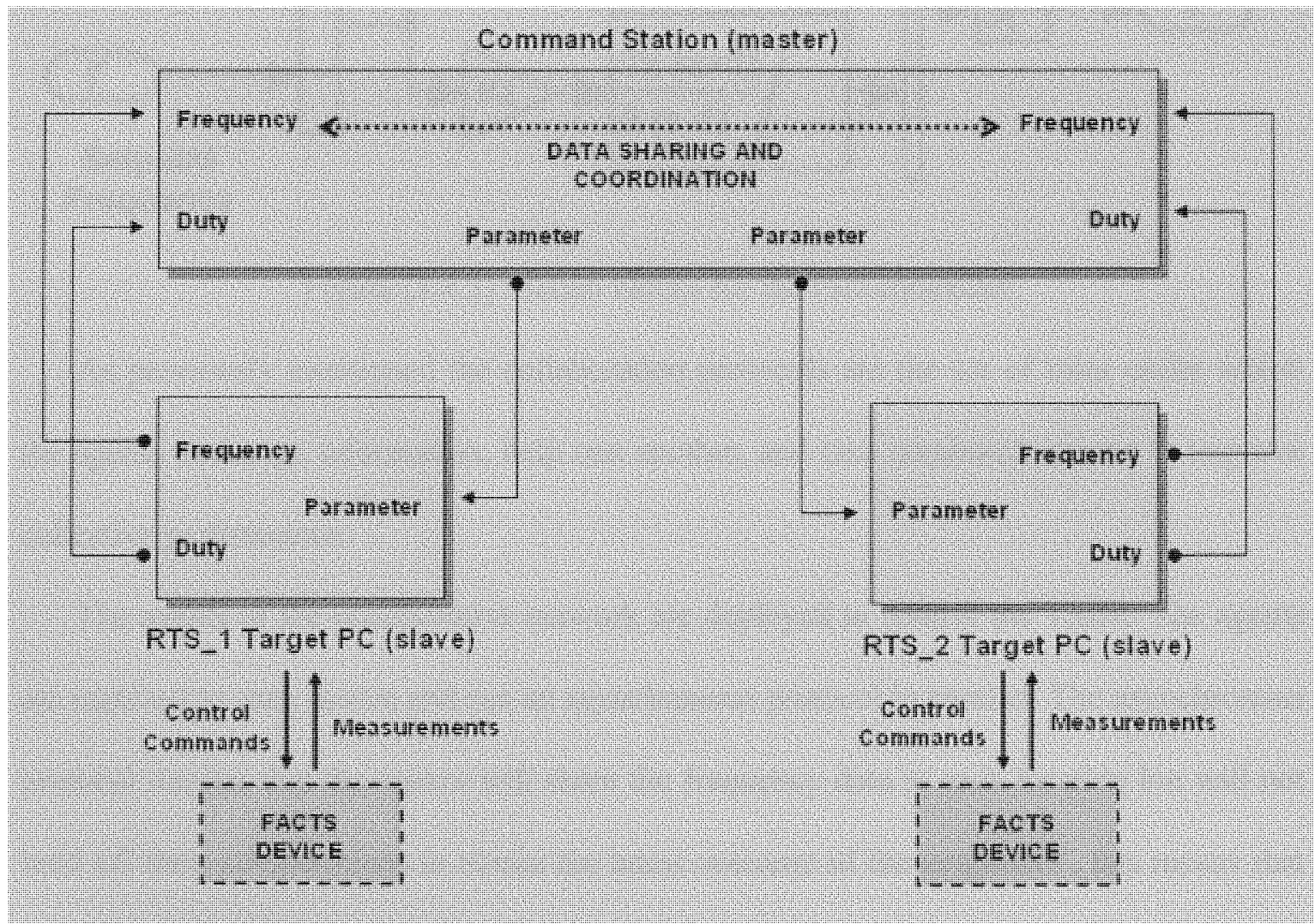

Figure (5.4.2) Real-time distributed control implementation for TSBR devices. 
The PID controller gives accurate and stable control and can often be adjusted without advanced mathematics. The parameters blocks for the PID controller are shown in Figure (5.4.3) the gains were set to the following set of values: Integral Gain $(\mathrm{Ki})=5$; Proportional Gain $(\mathrm{Kp})=1$; Sampling Time $(\mathrm{Ts})=0.00015$. The control signals are generated by Matlab (RT-Lab eviroment) and then sent to a control board that runs the TSBR. The frequency is sensed using a frequency transducer and then fed to one of the ADC channels of the Field Programmable Gate Array (FPGA) board.

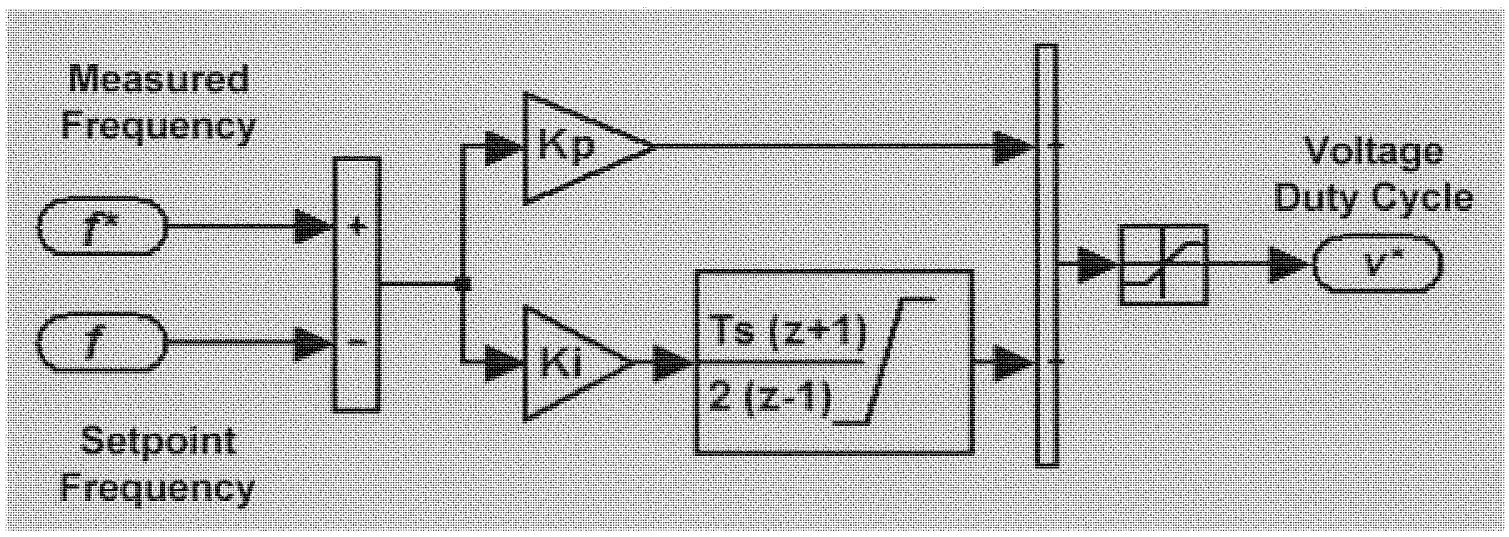

Figure (5.4.3) Matlab blocks for the PID controller.

As previously mentioned the collaboration protocols are the set of rules under which the agents exchange information, help one another, and take combined actions to improve collective performance.

The agents of the collaborative network are deployed to control the TSBR. The agents are matched to each device and run an asynchronous protocol in parallel. The asynchronous protocol implies that the agents will share the frequency data at each location but will not hold off their computations to obtain the latest outcome of the neighbors', this means that the agents work freely. This type of protocol is very significant for transient stability problems because the agents work in parallel and can respond quickly to disturbances. This 
type of protocol allows the agents to achieve the overall systems' objective function with minimum control. As mentioned before, the objective function in this case is the power balance of the generators, the electrical power equals to the mechanical power.

The details on the integration of software and hardware subsystem to implement realtime distributed control system are outlined in the following chapter. The evaluation and experimental results of the real-time distributed control implementation will be outlined in later chapters. 


\section{Chapter 6}

\section{Hardware and Software Integration for the Real-Time Control System}

This chapter outlines the digital real-time control with Hardware-in-the-Loop (HIL) system used as part of the software subsystem in the modeling of the FACTS devices on the power system simulator.

This tool was used with the aim of meeting the transient simulation needs of electronic power processors while solving the limitations of traditional real-time control. The power system simulator has two main subsystems: a software and hardware subsystem. The two subsystems were coordinated to achieve the real time control. The software subsystem includes Matlab toolbox and a $\mathrm{C}++$ compiler. The hardware subsystem includes a Field Programmable Gate Array (FPGA) data acquisition card, the corresponding control board, the sensors, and the desired equipment to be controlled. The use of a real-time control environment to achieve HIL simulation allows rapid prototyping, testing, emulation, and control strategies evaluation [16].

\subsection{Introduction}

An electrical network loaded with power electronics converters is a complex and nonlinear system. Consequently, performing system-level testing which is one of the major steps in developing a complex product in a comprehensive and cost effective way require real-time control with hardware in the loop.

One of the most demanding aspects for real-time control systems is to connect the inputs and outputs of the tested control system to a real-time simulation of the target process. As this implies that all control loops are closed via the simulator, this method is often called Hardware-in-the-Loop (HIL) simulation. By using the HIL simulations we can 
evaluate different subsystem interactions. A particular merit of this approach is that it even permits a gradual change-over from simulation to actual application, as it allows to start from a pure simulation and to gradually integrate real electrical and mechanical subsystems into the loop as they become available. HIL simulation can help reduce development cycles, cut overall cost, prevent costly failures, and test a subsystem exhaustively before integrating it into the actual operational system.

A multi-distributed modeling concept allows the simulation to run very fast and achieve real-time operation. In this approach, the global model is distributed over several processors through a communication link (i.e. TCP/IP, FireWire, Shared Memory).

Also, it allows the subsystems (components or group of components) to be executed at different update rates, cycles can be freed up for executing the subsystem(s) that need a faster updated. The multi-distributed modeling can involve different operating systems. Real time operating system (i.e. QNX, Linux) reduces considerably the simulation time requirement. This chapter describes the real time control environment used as part of the software subsystem of the power system simulator. Also, it outlines its software subsystem.

\subsection{Block Diagram and Schematic Interface}

The real-time control system is based on a real-time distributed simulation platform. It is optimized to run MATLAB in real-time, with fixed time step solvers, on a PC-Cluster. MATLAB is widely used in the dynamic and control system analysis as well as simulation areas.

MATLAB is the basic "numerical analysis engine" with add-on components called toolboxes. Simulink, a toolbox of MATLAB, is dynamic system simulation software that provides a convenient graphical user interface for building system models based on their 
equations. The Real Time Workshop (RTW) toolbox is capable of generating real time code for Simulink models. The real-time code is used to control a system in the HIL environment. The data generated by any of the system components, digital or hardware, can be displayed in real-time and/or saved for later use.

The system uses the Simulink as an interface for building, editing and viewing graphic models in block-diagram format. The block diagram models are converted into C-code using RTW, and then transferred in to target machine for real-time simulation. Figure (6.2.1) shows the real time simulator software subsystems architecture.

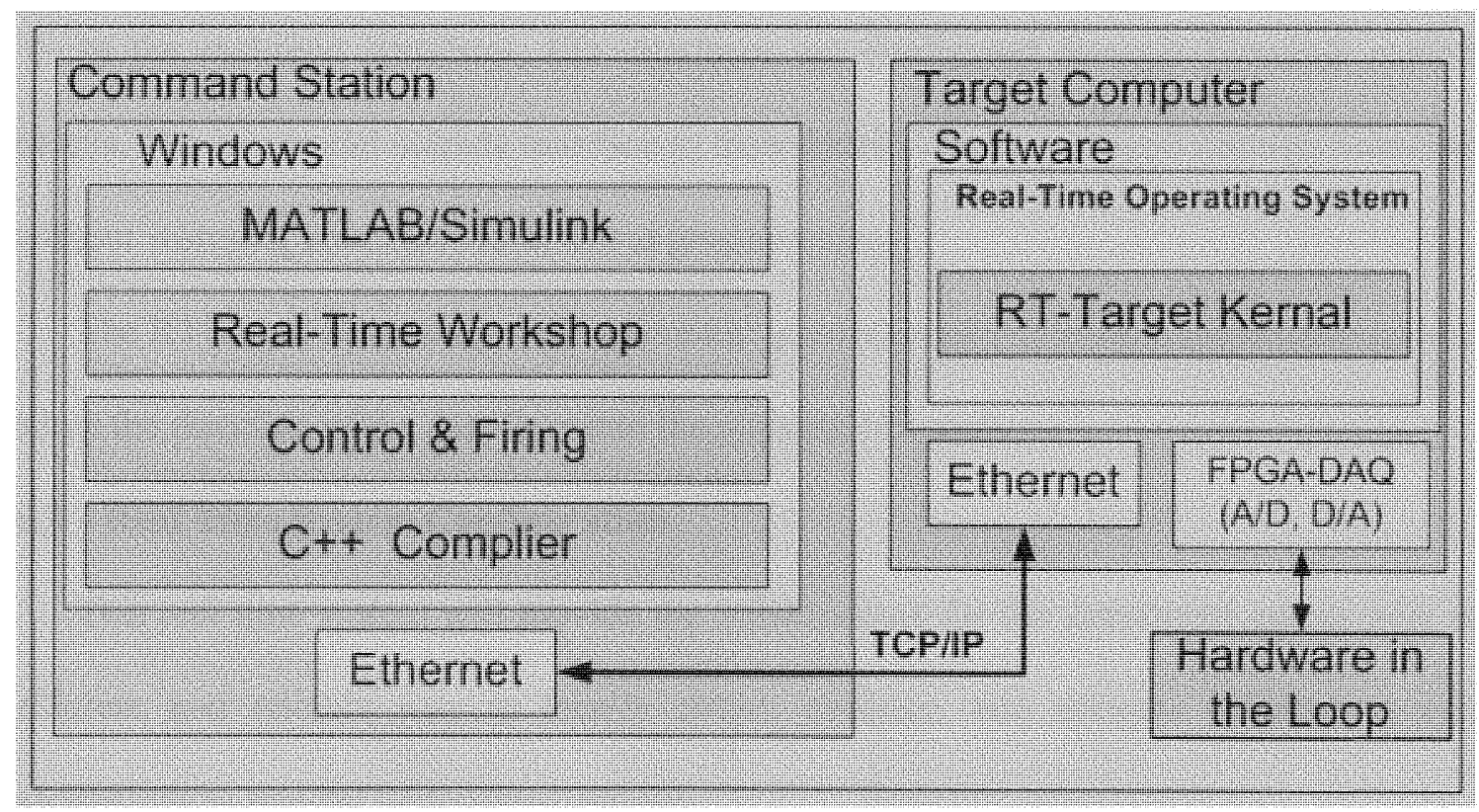

Figure (6.2.1) Host-target real time software subsystems architecture.

The target computer or real-time station (RTS) utilizes a real-time operating system QNX that allows the PC to run real-time simulation with HIL. The target computer is interfaced with the equipment through a Field Programmable Gate Array (FPGA) card for data acquisition a control. The FPGA card is acquiring data from the sensor and sending 
control decisions to the equipment. The target $\mathrm{PC}$ is also sending and receiving data from de master PC through TCP/IP interconnection protocol.

A major requirement for real-time HIL applications is the interfacing with real world hardware devices, controller or physical plant. The DAQ (I/O) provides the interface between the target computer and the HIL system. High performance I/O Xilink Virtex-II pro FPGA DAQ was used. This FPGA DAQ card can run controlled inverter with AC-side diode rectifier at time step of 10 microseconds. This allowed the desire fast control response necessary to damp transient oscillation in the system.

In the real-time simulator, $\mathrm{I} / \mathrm{O}$ interfaces are configured through Simulink custom blocks. The inputs and outputs interface to these blocks can be managed through Simulink, without worrying about low-level driver programming.

The RT-Simulator manages the automatic generation of $\mathrm{I} / \mathrm{O}$ drivers and models code to allow the direction of the data flow into the physical I/O cards.

\subsection{Real Time System Configuration}

This real time system will provide the infrastructure for the distributed agents with an efficient and reliable way to locally detect, evaluate, and mitigate different system oscillations to prevent widespread outages.

Figure (6.3.1) shows the configuration of the real time simulator that consists of:

- One or more target PC's (computation nodes); one command station to manage the communication between the hosts and the targets and the communication between all other target PC's. The targets use the QNX real-time operating system or Linux; 
- One or more host PC's allowing multiple users to access the targets; one of the hosts has the full control of the simulator, while the other hosts, operating in readonly mode, can receive and display signals from the real-time simulator;

- I/O's of various types (analog input and output, digital input and output, PWM input and output, timers, encoders, etc). The I/O's can be managed by dedicated processors distributed over several nodes.

The real-time simulation environment uses Ethernet connection $(100 \mathrm{Mb} / \mathrm{s})$ to communicate between the host and the target PC. The Ethernet connection between the target nodes allows parallel computation of models with small time step size. Full control of the target application and the computer is provided to the user through the RT-shell [16].

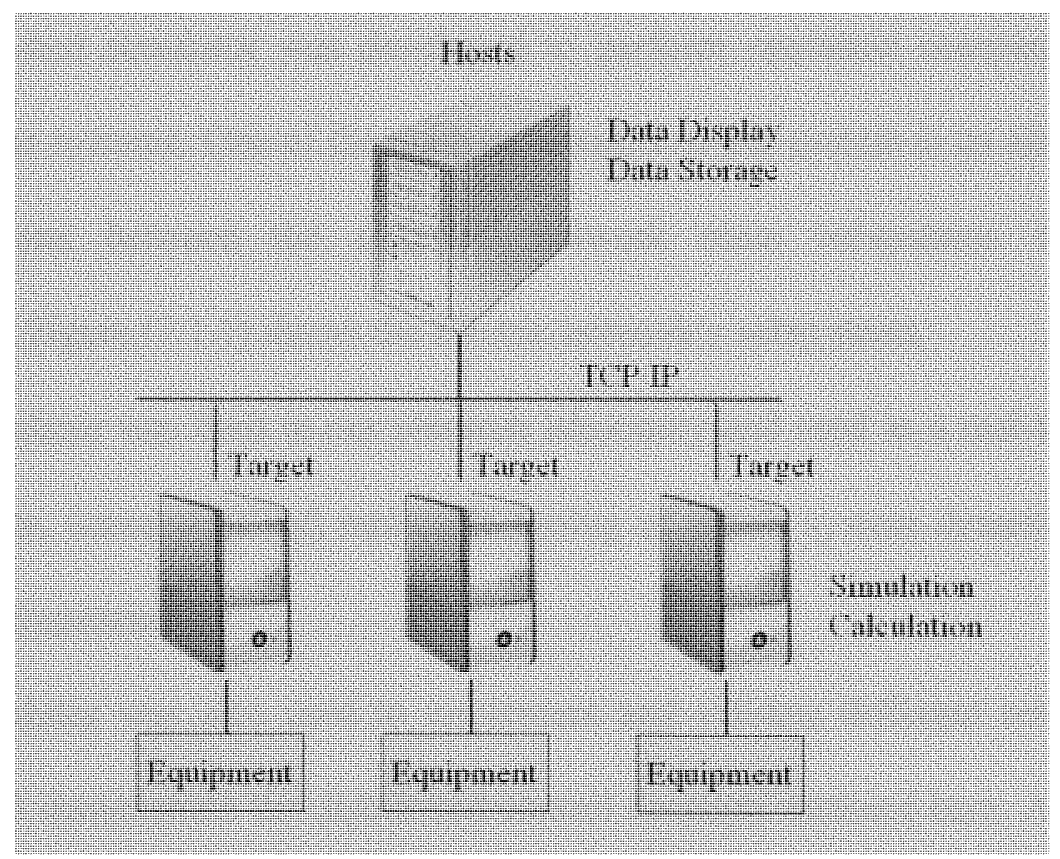

Figure (6.3.1) Configuration of the real time simulator

This chapter outlined the real time tools and configurations used in the development of the power system simulator with HIL. 


\section{Chapter 7}

\section{Test-bed System Development and Integration}

This chapter presents the development of the hardware test-bed system capable of performing the operation of modern power system infrastructure. The design and construction of the test-bed system as well as its implementation to simulate the distributed control scheme is given.

Some important elements of the system incorporate real life, flexible power system network hardware and data acquisition (DAQ) system to evaluate the issues that could trigger cascaded failures.

\subsection{Introduction}

This test-bed simulator was developed to perform operation comparable to that of modern power systems. One of the main advantages of this system is its ability to emulate the interactions between the electrical network main subsystems in a real-time environment. Figure (7.1.1) shows the electric network's subsystems.

This simulator was built in our research laboratory and used as a real-time test-bed to allow rigorous, transparent and replicable testing of power system dynamic responses to different contingencies.

The simulator was also used to implement and validate different corrective control strategies and demonstrate the distributed control concepts outlined here. A real-time testbed system can improve development time of new infrastructures, avoid expensive malfunctions, reduce cost, and rigorously test the system before its implementation and eventual utilization in an operational system. 


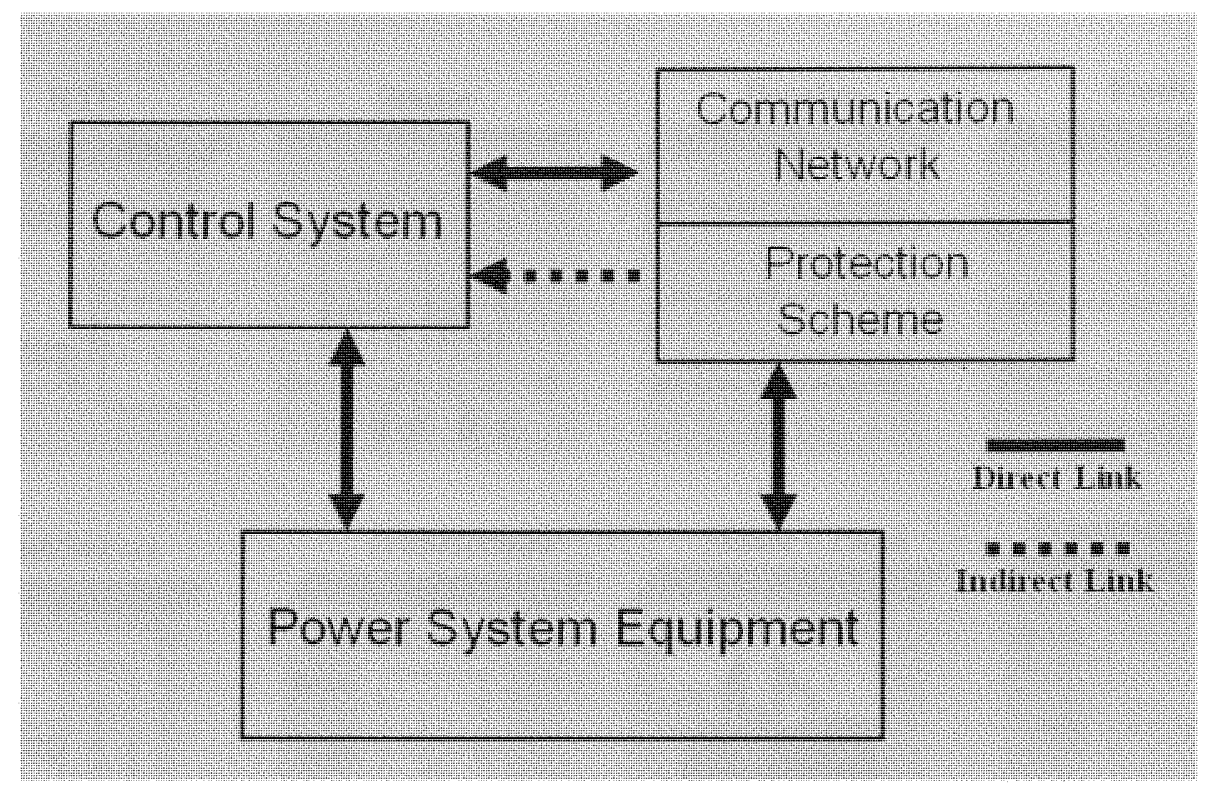

Figure (7.1.1) Power system four main subsystems.

This chapter provides the system's description and scaling method. The hardware subsystem includes existing laboratory equipment in addition to newly designed components. The implementation of the software sub-system and control scheme is also presented..

\subsection{System Description and Scaling}

The power system simulator contains different equipments and components such as generators, buses, transmission lines, protective devices (e.g. Breaker, relays, fuses), current transducers, voltage transducers, linear loads, nonlinear loads and, FACTS devices. Equipments and components are set up as building blocks.

The building block scheme allows a flexible system configuration and the representation of different operating conditions. Figure (7.2.1) shows the building blocks for various system components. The system was designed in this way to allow rapid prototyping and building flexibility. Buses and transmission line models; as well as, sensor, static loads and motors are ser in this building block scheme. 


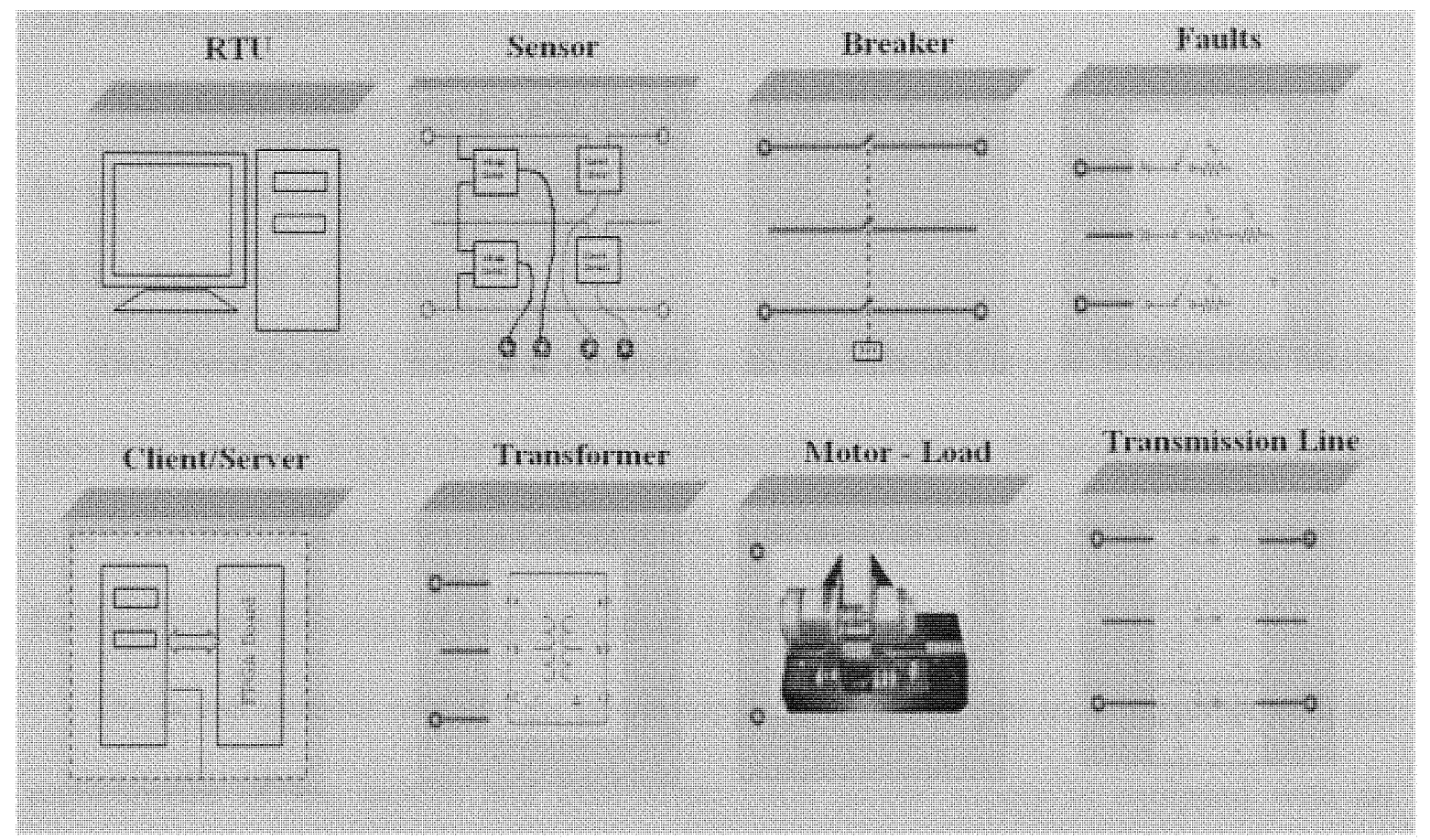

Figure (7.2.1) Scheme for components' blocks.

The power system simulator is a three phase system simulator operating at a $2 \mathrm{kVA}$, $208 \mathrm{~V}$. This simulator was originally designed to model the scale up IEEE 9-bus test system. The system was scaled down by using the voltage transformer VT's and the current transformer (CT's) ratios as in equation (7-1).

$$
\mathrm{Z}_{\mathrm{sec}}=\frac{\mathrm{R}_{\mathrm{CT}} * \mathrm{Z}_{\mathrm{pri}}}{\mathrm{R}_{\mathrm{vT}}}
$$

Where $Z_{\text {sec }}$ is the impedance in the secondary side in ohms that represents the scaled impedance using the VT's and CT's turns ratio, $Z_{\mathrm{pri}}$ is the impedance of the primary in ohms. Here, $R_{V T}$ and $R_{C T}$ are the $V T$ turns ratio and $C T$ current ratio, respectively. This method allows the rapid scaling of the system component such as transmission lines. Basically, the system is scaled referred to the low side of the voltage and current transformer. 


\subsection{Hardware Sub-System}

This section reviews design and development of the test-bed components; as well as, the existing equipment in the laboratory.

\subsubsection{Test-Bed Equipment}

The Power System Test-bed is equipped with two sets of Console Workstation Model shown in Figure (7.3.1.1). The laboratory's workstations provide the necessary components to perform motors and generators experiments, also serve as versatile power and instrument facilities for a wide range of other laboratory experiments.

The power console contains six power supplies, whose ranges are compatible with the requirements of the motors and generators. Each power supply is equipped with an indicating pilot light, a magnetic circuit breaker to protect against overloads, and 25ampere color-coded output jacks.

The ratings of the power supplies are as follows:

- Fixed 120V.AC, 1-phase, 2-wire, 6A;

- Fixed 120/208V.AC, 3-phase, 4-wire, 6A;

- Variable 140/0-240V.AC, 3-phase, 4-wire, 6A;

- Variable 0-150V.DC, 1.0A, isolated, 1\% rms;

- Variable 0-125V.DC, 5.0A, isolated, $1 \% \mathrm{rms}$;

The metering console contains all of the instruments provided in the three separate meter panels, AC Voltmeter-Ammeter, DC Voltmeter-Ammeter, and AC Wattmeter.

Input power required is $120 / 208 \mathrm{~V} . \mathrm{AC}-3$-phase - 5W - 15A. Each workstation unit is provided complete with cords [16]. 


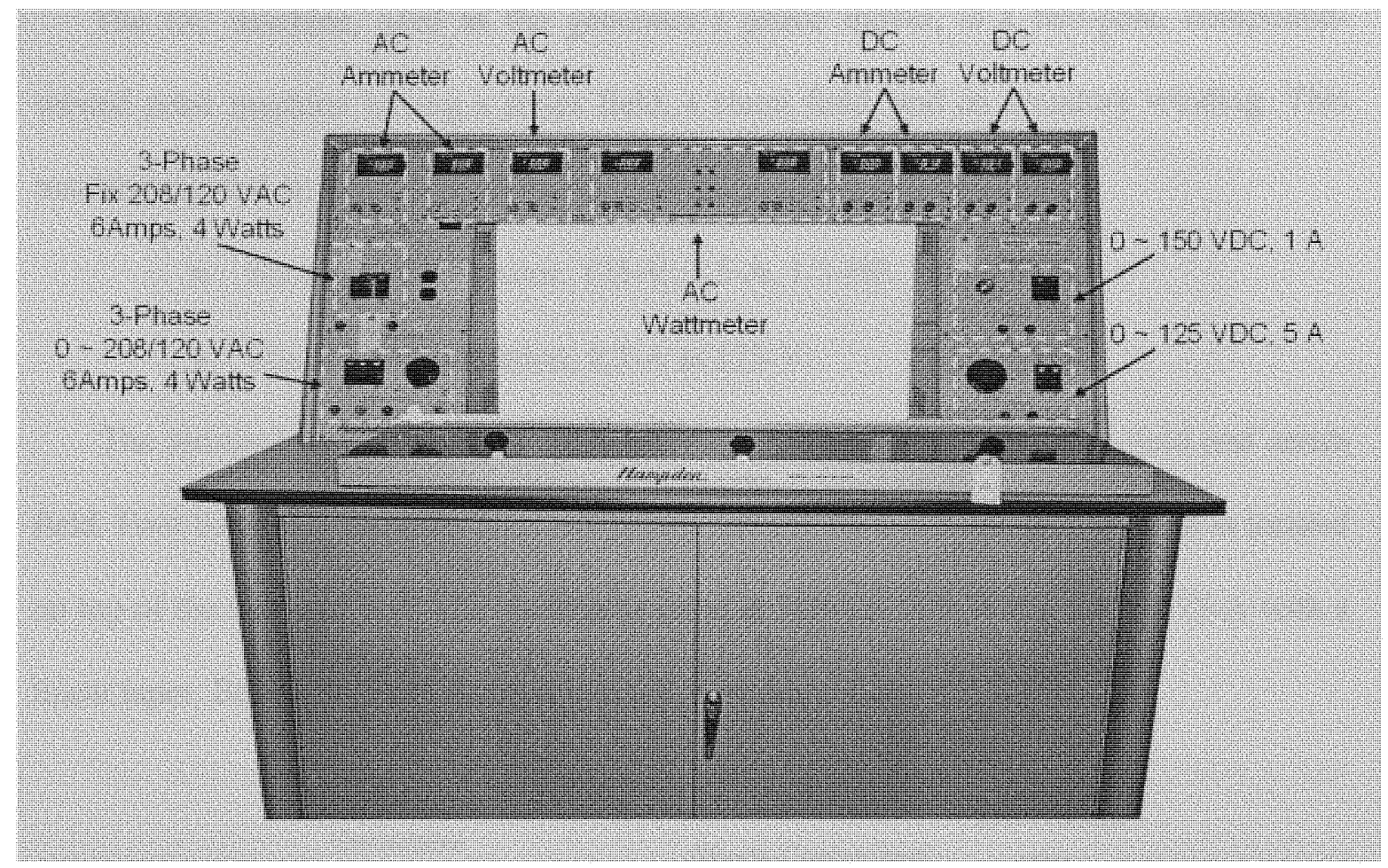

Figure (7.3.1.1) Supply and metering console workstation.

The workstation also contains three phase transformer set up, motors, generators, resistive loads, reactive loads, capacitor banks, resistor banks and inductance banks. These equipments are used as required, based on the selected experiment.

The motor-generator set up shown in Figure (7.3.1.2) consists of one synchronous alternator coupled with a DC motor. The synchronous alternator is a three-phase, one-third horsepower, rated at $1750-\mathrm{RPM}$, and $208 \mathrm{~V}_{\mathrm{LL}}$ at $60 \mathrm{~Hz}$. The machine is mounted behind a terminal box which consists of two ports for field connection to the rotor and six ports for three-phase connection to the stator. It also has six circuit breaker terminals and a circuit breaker switch. A toggle switch enables the user to switch the field connection for induction starting or synchronous run [17]. 
The DC machine, used as the generator's prime mover, is a two-pole, compoundwound rotor machine having a series field, a shunt field, and an armature winding. This machine is rated at:

- Shunt or Series motor: 125-V, 3.5-A, 1/3-HP, 1800-RPM;

- Shunt Field Excitation: 0.5-A at 125-V;

The terminal box includes terminal pairs for the field rheostat, shunt field and series field. Four terminals comprise the start switch and the knob for the field rheostat is located on top of the terminal box. The rheostat is rated at $300-\Omega, 100$-Watts, and $0.575-\mathrm{A}$ with a linear taper. Below the field rheostat is a 1-A slow-blow fuse.

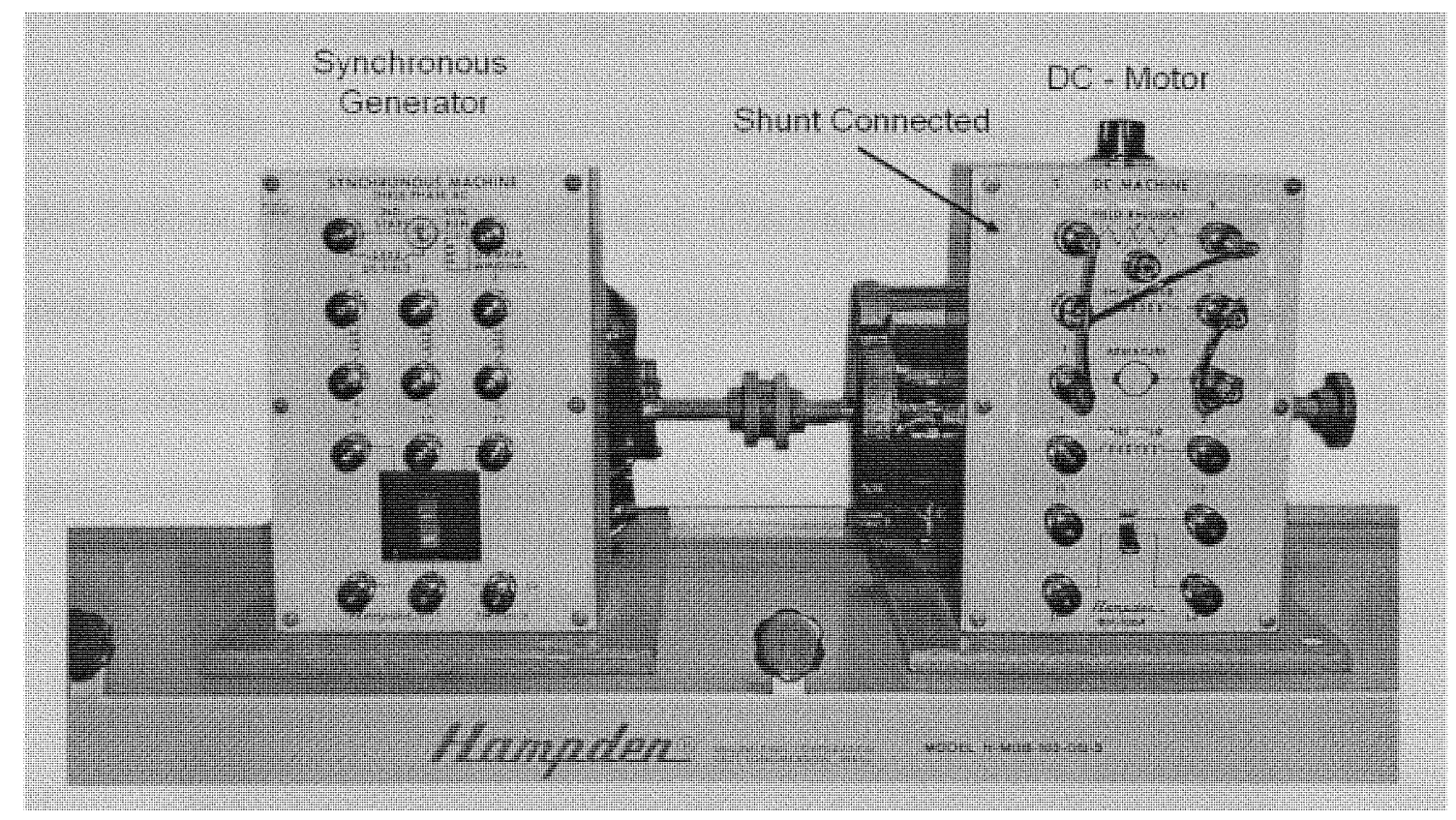

Figure (7.3.1.2) Motor-generator set-up.

The three-phase transformer in Figure (7.3.1.3) is made up of three single-phase transformers with multiple primary and secondary taps. Each transformer may be used separately or combined for three-phase wye or delta configuration. Each transformer is rated at $120-\mathrm{VA}$ at $60 \mathrm{~Hz}$. 


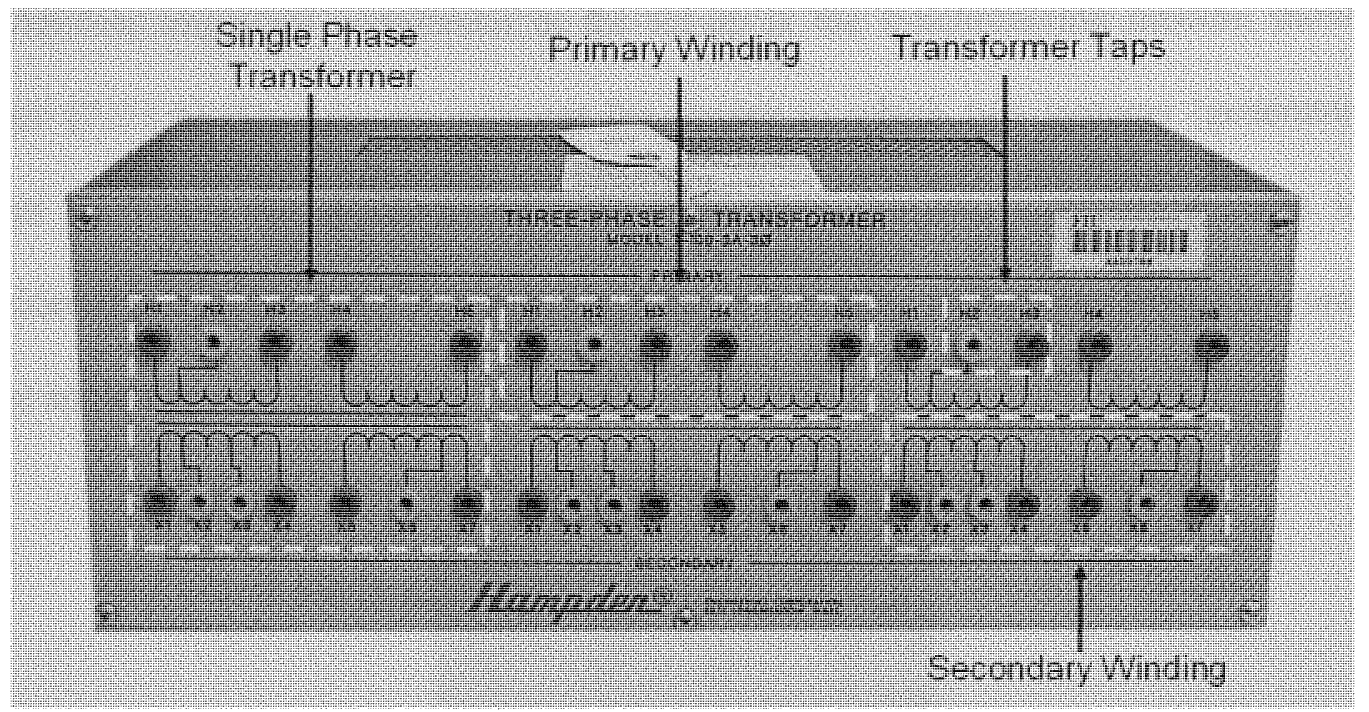

Figure (7.3.1.3) Three-phase transformer.

The resistance-reactance load device has three variables, resistance, capacitance and inductance. The value of the resistance is controlled by six toggle switches each of which adds $2000-\Omega$ in parallel with each other. The reactance loads are controlled by related knobs which serve to adjust the power factor of the load for either lagging (inductive) or leading (capacitive). The entire load may be used as single or three-phase applications for purely resistive, purely reactive or combined loads. See Figure (7.3.1.4).

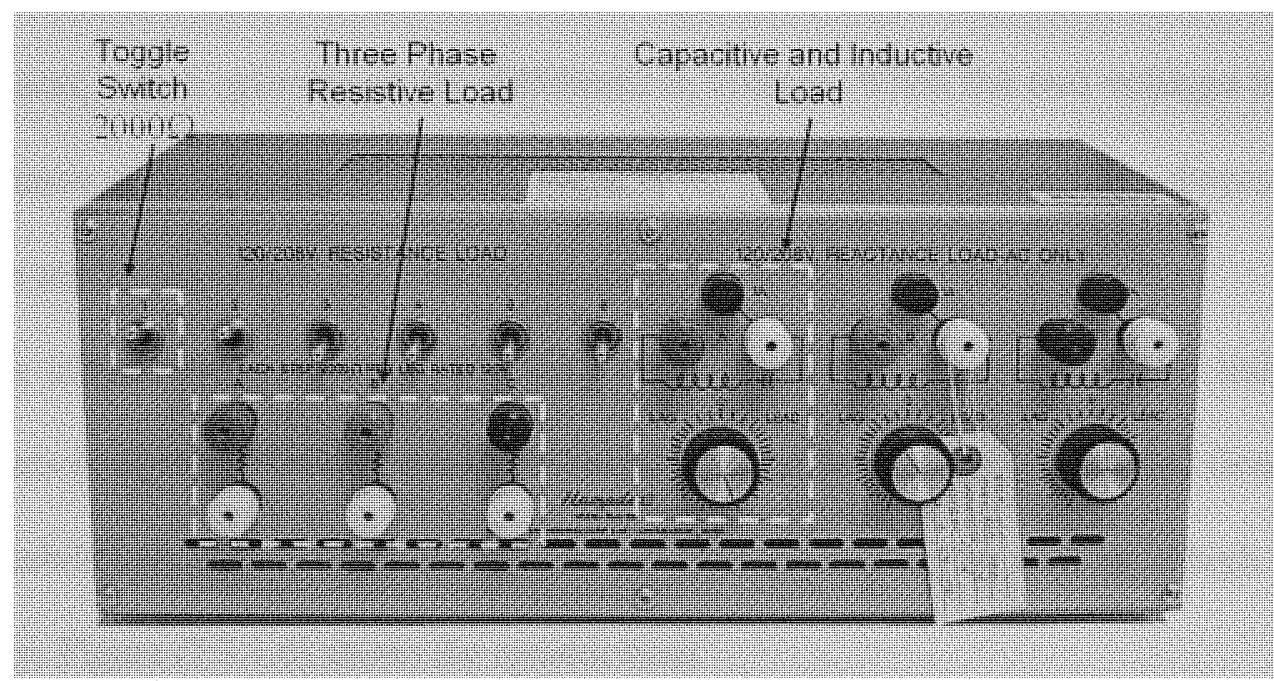

Figure (7.3.1.4) Resistance-reactance load. 
Another important component in the set-up is the synchronizing module shown in Figure (7.3.1.5). This device indicates the degree to which two systems (generators or power networks) are synchronized with each other. For two electrical systems to be considered in synchronism, both systems must be operating at the same frequency, and the phase angle between the systems must be zero.

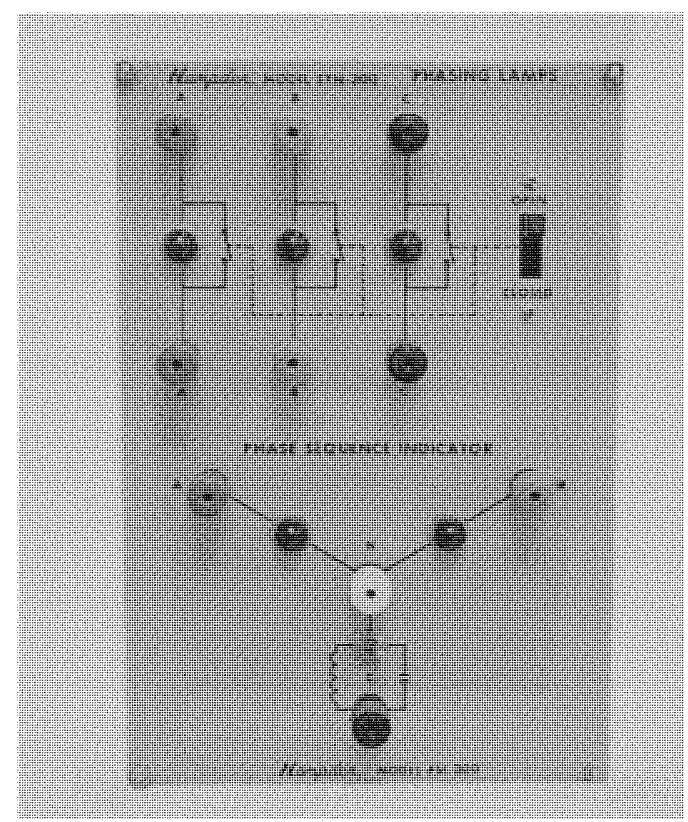

Figure (7.3.1.5) Synchronizing module.

The synchronizing module consists on a three-pole switch with a lamp across each pair of contacts, which operates on the difference in voltage. When the two voltages have the same phase sequence and frequency, there is no difference between the voltages at any point in any of the cycles. Refer to Appendix B.

The laboratory is also equipped with mobile resistance, capacitance and inductance load carts designed to provide a suitable resistance load for use with $\mathrm{AC}$ or DC Generators. This mobile cart allows flexible configuration into the system; as well as, a high loading capacity. Figure (7.3.1.6) shows the resistor bank. 
This resistor bank is utilized to represent very large loads into the power system; the resistor bank is rated at:

- 4.2-kW, 208/120-V.AC, for three-phase with four wires;

- Maximum power per phase is $1.4-\mathrm{kW}$ at $120-\mathrm{V}$;

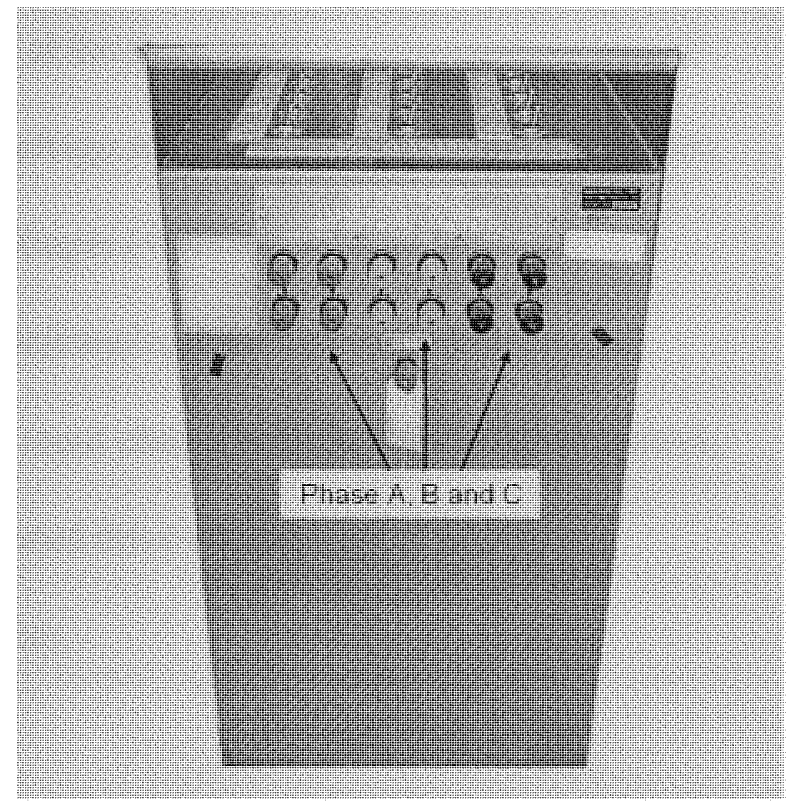

Figure (7.3.1.6) Resistance load cart.

\subsubsection{Power Electronic Converter Board}

The test-bed hardware also includes power electronic equipments. Figure $(7.3 .2 .1)$ shows the power electronic converter board and Figure (7.3.2.2) shows the circuit layout for the converter. The converter board consists of two independent three phase inverters. Each 3-phase inverter uses MOSFETs as switching devices. The converter board is used to perform a variety of experiments including $\mathrm{DC} / \mathrm{DC}, \mathrm{AC} / \mathrm{DC}, \mathrm{AC} / \mathrm{AC}$ and $\mathrm{AC}$ and $\mathrm{DC}$ machine drives, FACTS, and actuators.

The power electronic converter board is utilized in a real-time distributed simulation platform. It is optimized to be run by Simulink and SimPowerSystems in real-time, with 
fixed time step solvers [18]. This converter board can be configured to be used for more than one conversion stage; which makes it a real-time power processor.

The main features of the converter board are:

- Two independent 3-phase PWM converters for complete simultaneous control of two machines at the same time;

- Current and voltage sensors with wide range capabilities;

- Use of low voltages $(<50 \mathrm{~V})$ to achieve low cost;

- Complete digital/analog interface with FPGA board;

- Digital PWM input channels for real-time digital control;

- On-board isolated drive circuit;

- Fault protection;

- Output filter;

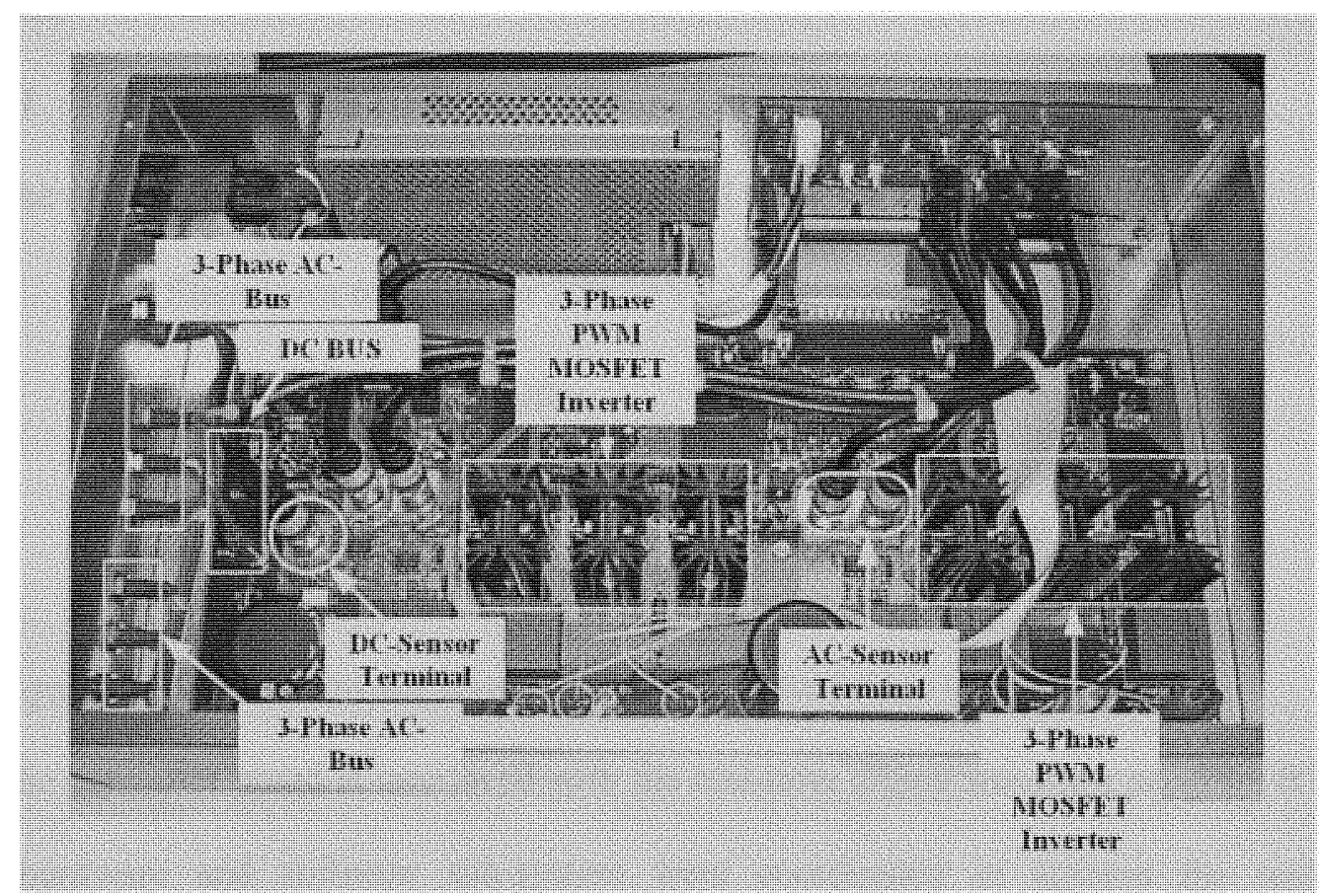

Figure (7.3.2.1) Power electronic converter board. 


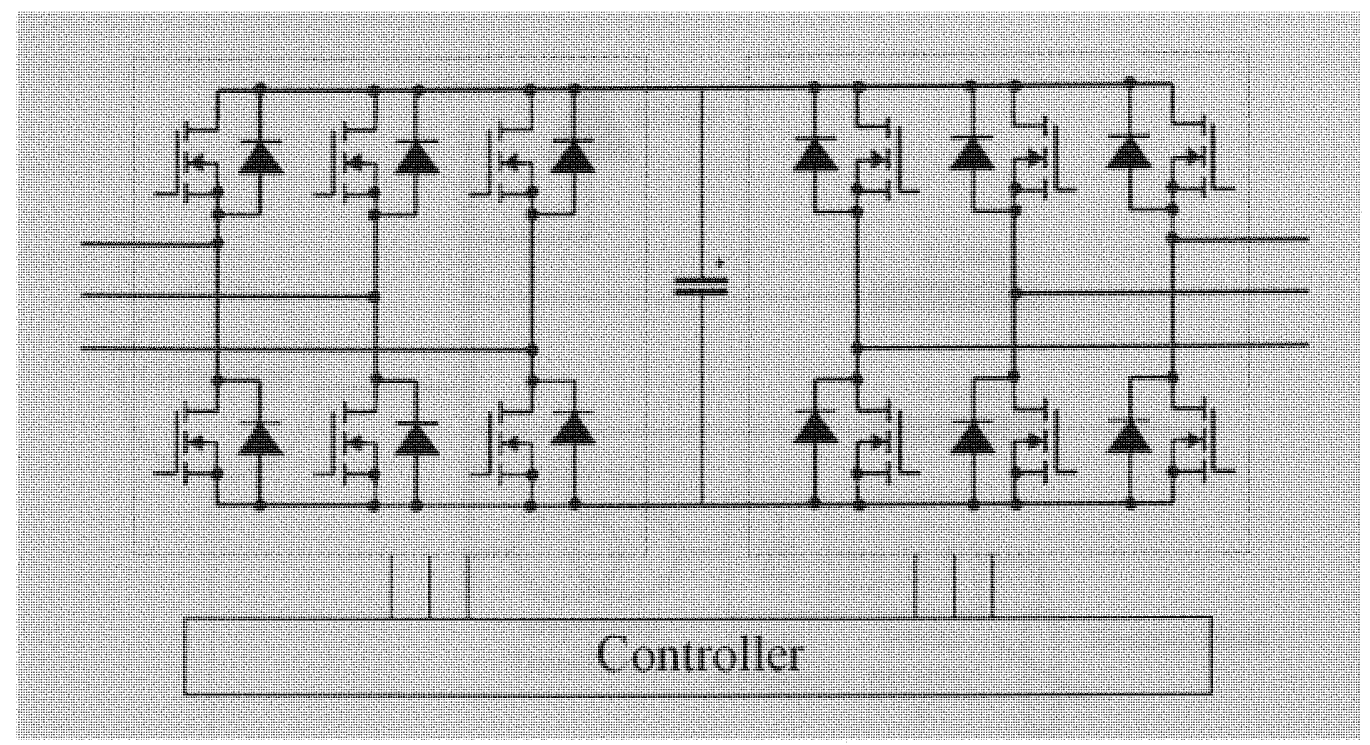

Figure (7.3.2.2) Circuit layout for the converter.

As discussed earlier, the electronic power processor such FACTS devices can be used for direct control of some parameters of the power systems offering the required controllability over the power flow. This power electronic converter board is used to model a TSBR.

\subsubsection{Thyristor Switch Braking Resistor (TSBR) Model}

The physical model of the TSBR is developed using the following components:

- A Step-down transformer $208 / 30 \mathrm{~V}_{\mathrm{LL}}$;

- A three-phase diode rectifier;

- A power electronic converter board;

- A resistor bank;

Figure (7.3.2.1.1) show the layout of the TSBR attached to the generator bus.

The converter board is interfaced with the real-time digital simulator program RT-lab. The hardware and software sub-system were coordinated to achieve the real-time simulation. 


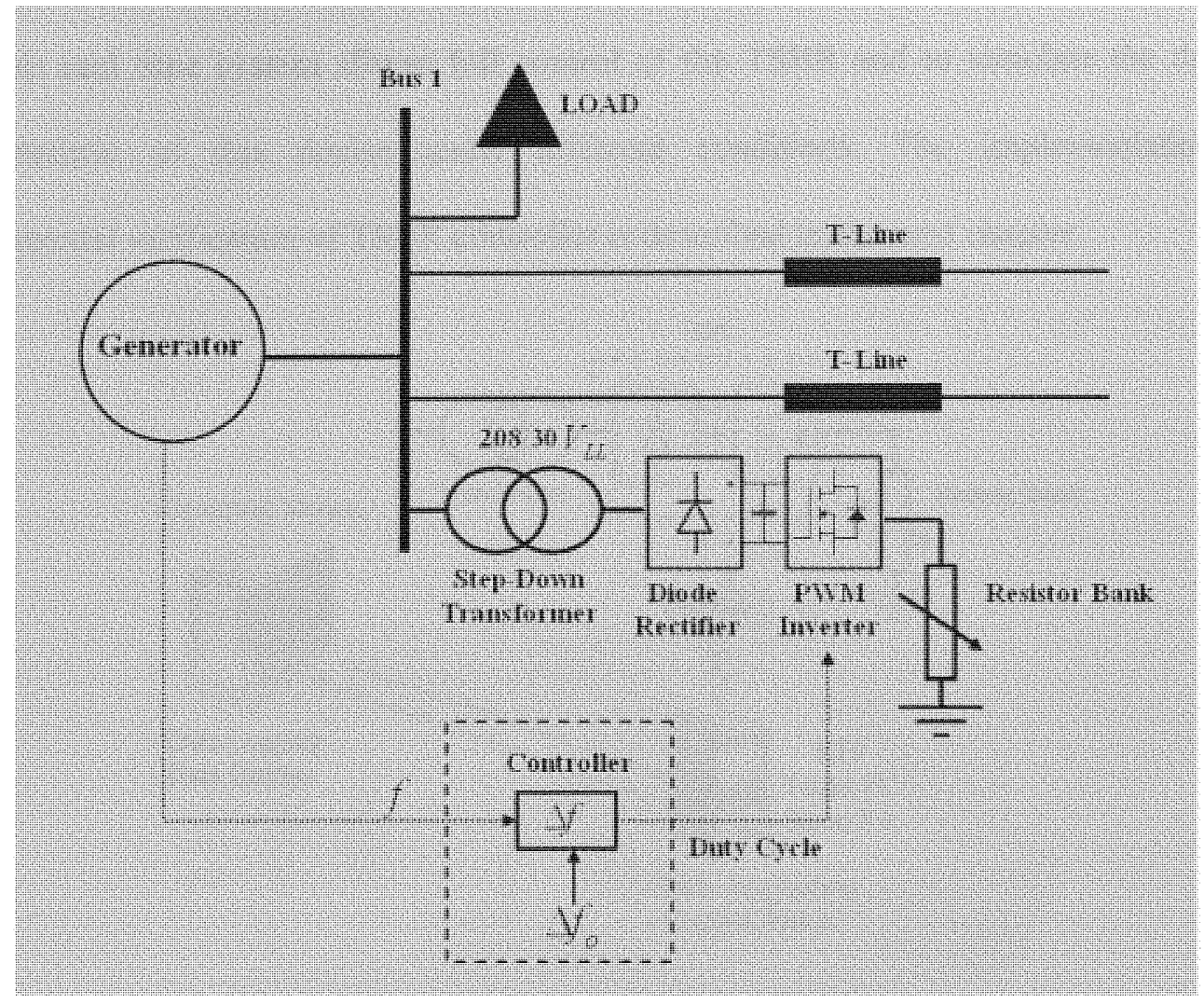

Figure (7.3.2.1.1) Layout of the TSBR attached to the generator bus.

This real-time environment simulation is used to test and evaluate the control strategies of the TSBR. A schematic view of the RT-lab Real-Time Station (RTS) is shown in Figure (7.3.2.1.2). The RTS consist of, a PC running on real-time operating system RT-QNX. This $\mathrm{PC}$ has a dual-Xeon processor of $3.4 \mathrm{GHz}$ and interface to the power converter board through a FPGA card. This FPGA DAQ card can run HIL control at time step of up to 10 microseconds. The simulation data is then send to a command PC for data logging and display.

The simulator uses Ethernet connection $(100 \mathrm{Mb} / \mathrm{s})$ to communicate between the host and the target PC. The Ethernet connection between target nodes allows parallel computation of models with small time step size. 
This real time system allows full control of the TSBR and to simulate the dynamic characteristic of this devise under different conditions. The implementation and experimental verification of TSBR controller is described in the next chapter.

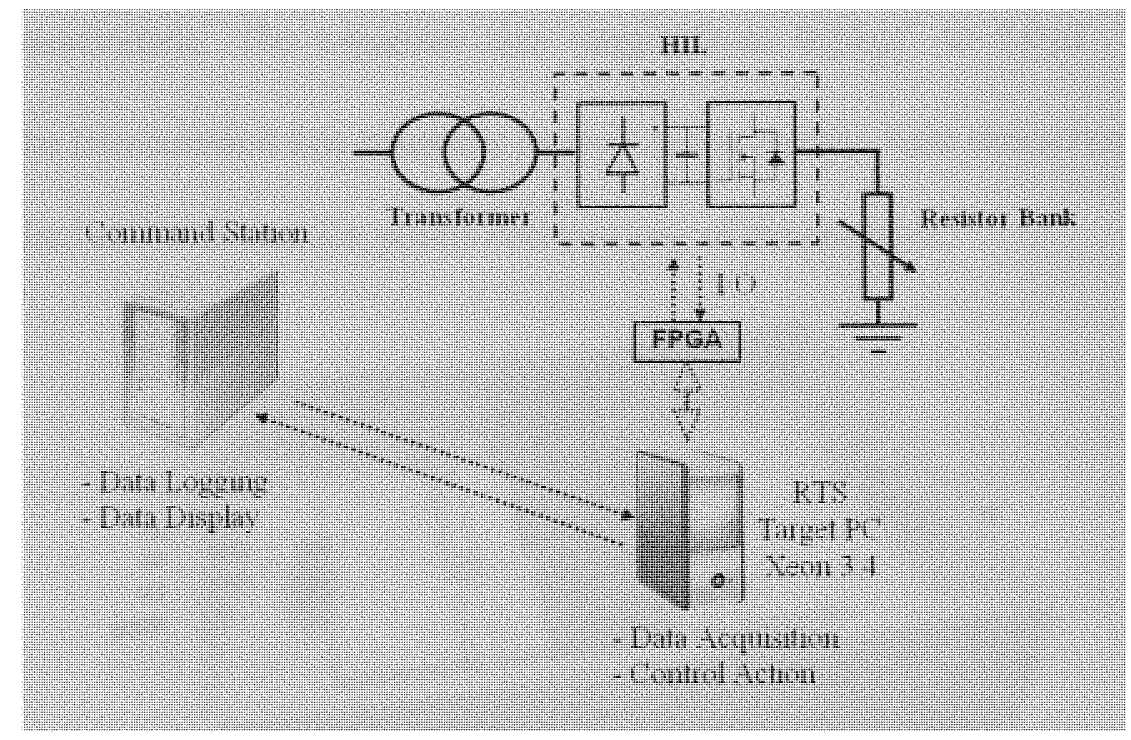

Figure (7.3.2.1.2) Schematic of the (RTS) and the command station.

\subsubsection{Development of the Transmission Line Model}

The transmission line model is a three phase transmission line simulator operating at 208 volts. This line model has been built and used to simulate lines of up to 300 miles in length, and was originally designed to model a $230 \mathrm{kV}$, four wire (3-phase and neutral) transmission line.

The transmission line model consists of various modules that can be interconnected in different configurations depending on the system being modeled. The transmission line model is constructed using lumped parameters for the resistance, the inductance, and line to ground capacitances. The designed prototype diagram for the transmission line model is shown in Figure (7.3.3.1). 
The basic line impedance module consist of three $2.5 \mathrm{ohm}$ at $60 \mathrm{~Hz}$ air core inductors with fixed shunt capacitance. Figure (7.3.3.2) and Figure (7.3.3.3) show two different sets of transmission line models built in the laboratory. The shunt capacitance is set to match the line characteristic. The capacitor size ranges from $0.25 \mu \mathrm{f}$ to $3.0 \mu \mathrm{f}$. The model represents the transmission line magnetic as well as the electric field characteristics. Faults may be applied at the connection points between any of the modules.

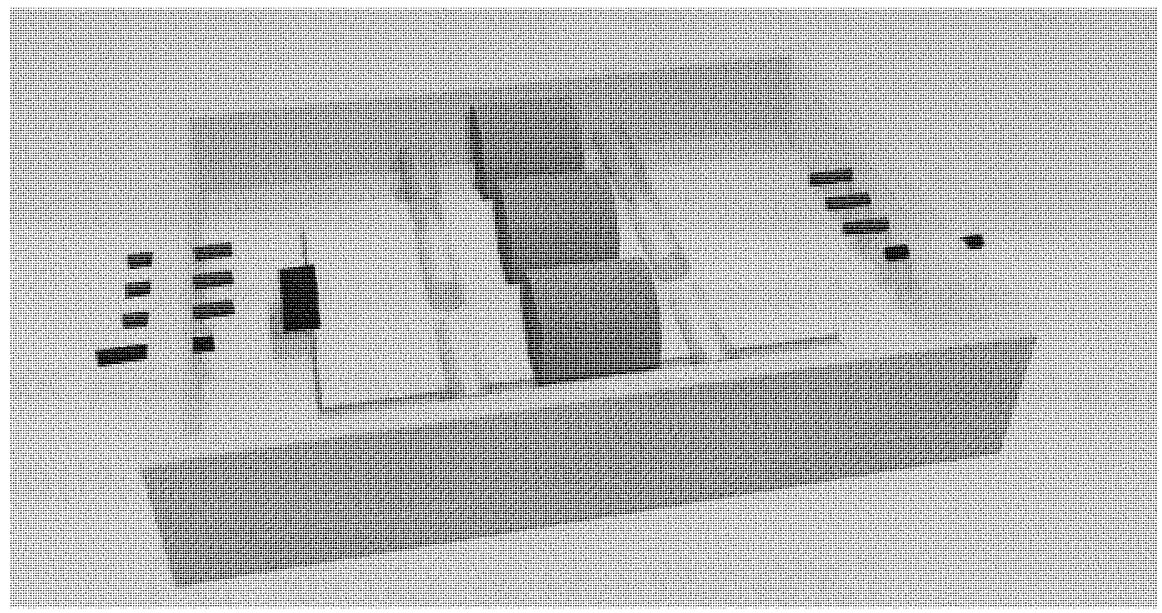

Figure (7.3.3.1) Prototype schematic for the transmission line model.

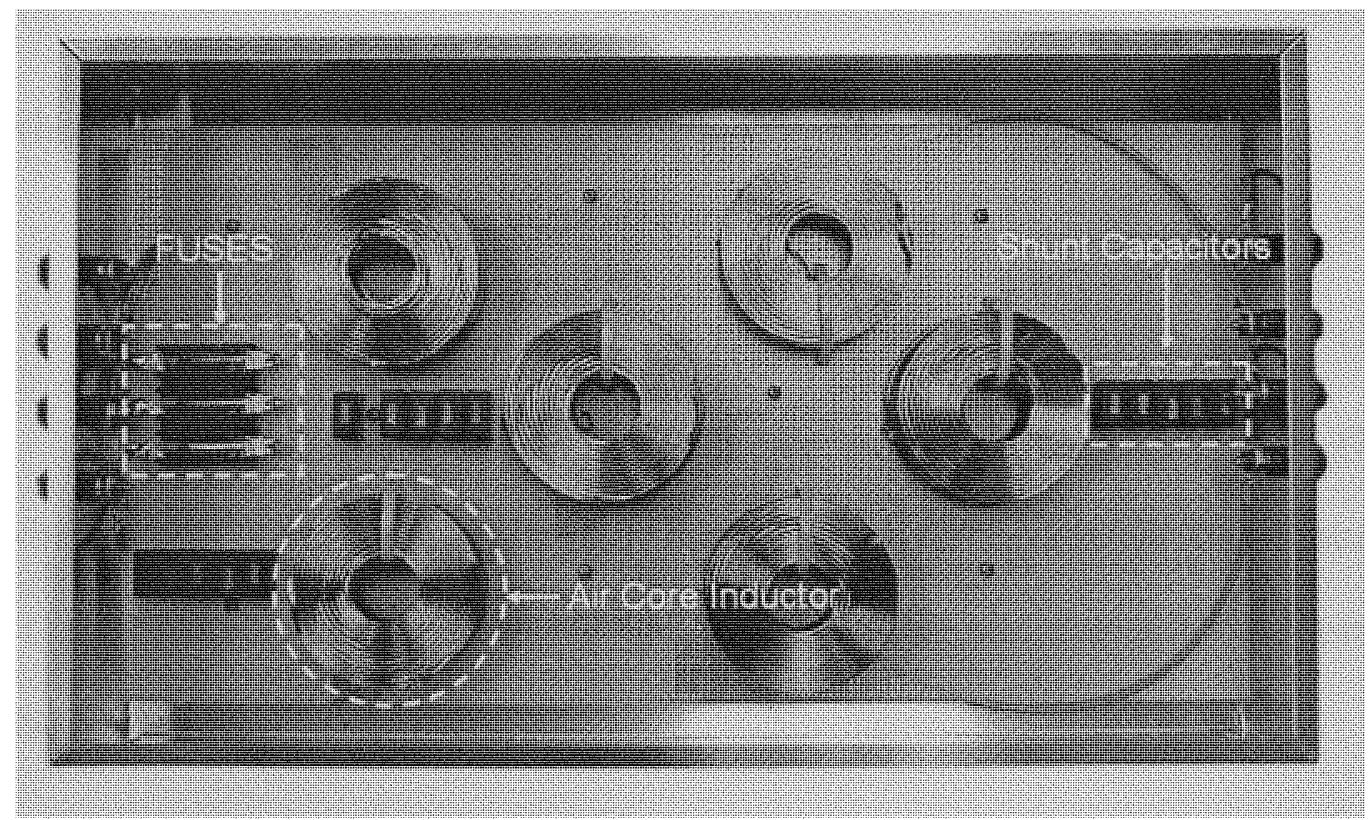

Figure (7.3.3.2) Transmission line model for 150 miles. 


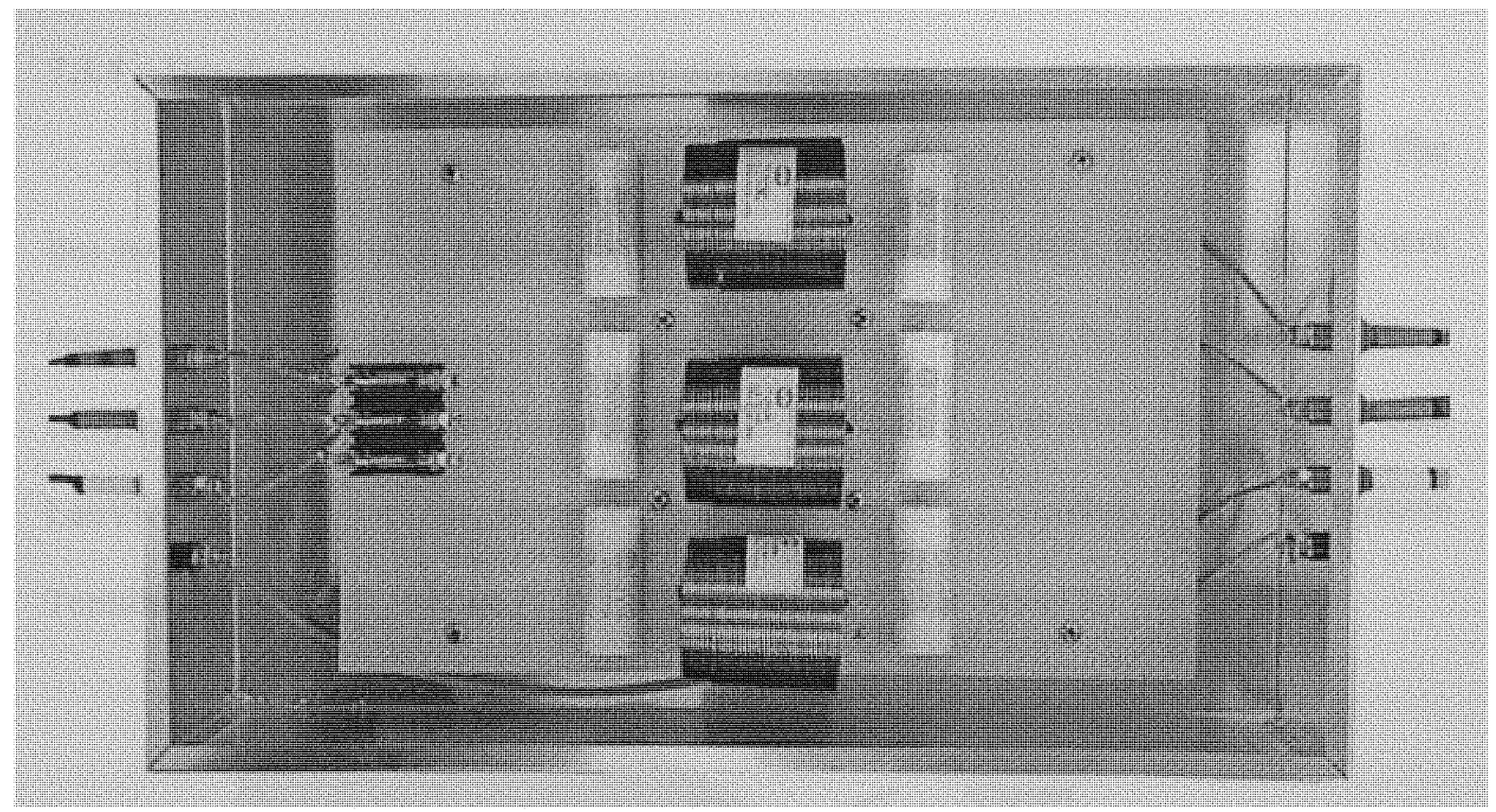

Figure (7.3.3.3) Transmission line model for 70 miles.

The ABDC parameters for the transmission line model are represented as a matrix in equation (7-2) [19].

$$
\left[\begin{array}{c}
\mathrm{V}_{\mathrm{S}} \\
\mathrm{I}_{\mathrm{S}}
\end{array}\right]=\left[\begin{array}{cc}
\left(1+\mathrm{YZ}_{1}\right) & \left(\mathrm{Z}_{1}+\mathrm{Z}_{2}+\mathrm{YZ}_{1} \mathrm{Z}_{2}\right) \\
\mathrm{Y} & \left(1+\mathrm{YZ}_{2}\right)
\end{array}\right]\left[\begin{array}{l}
\mathrm{V}_{\mathrm{R}} \\
\mathrm{I}_{\mathrm{R}}
\end{array}\right]
$$

Where the parameters $\mathrm{ABCD}$ are as follows,

$$
\begin{gathered}
A=D=\left(1+Y Z_{1}\right) \text { in per-unit } \\
B=\left(Z_{1}+Z_{2}+Y Z_{1} Z_{2}\right) \text { in ohms } \\
C=Y
\end{gathered}
$$

Where $\mathrm{A}, \mathrm{B}, \mathrm{C}$ and $\mathrm{D}$ are de parameters that depend on the transmission-line constants $\mathrm{R}, \mathrm{L}, \mathrm{C}$ and $\mathrm{G} . \mathrm{ABCD}$ parameters can be used to described the variation of the line voltage with the line loading. 


\subsubsection{Development of the Bus-Bar Model}

Another important component of the hardware development is the bus-bar model. The bus model consists of a module that contains switching devices, measurement devices (sensors) and data acquisition (DAQ) terminals. This module is then interfaced to a computer through a data acquisition card from National Instruments. The computer uses LabView software package, for data display, assessment and control; acting as a Remote Terminal Unit (RTU). Figure (7.3.4.1) shows the prototype model for the bus-bar.

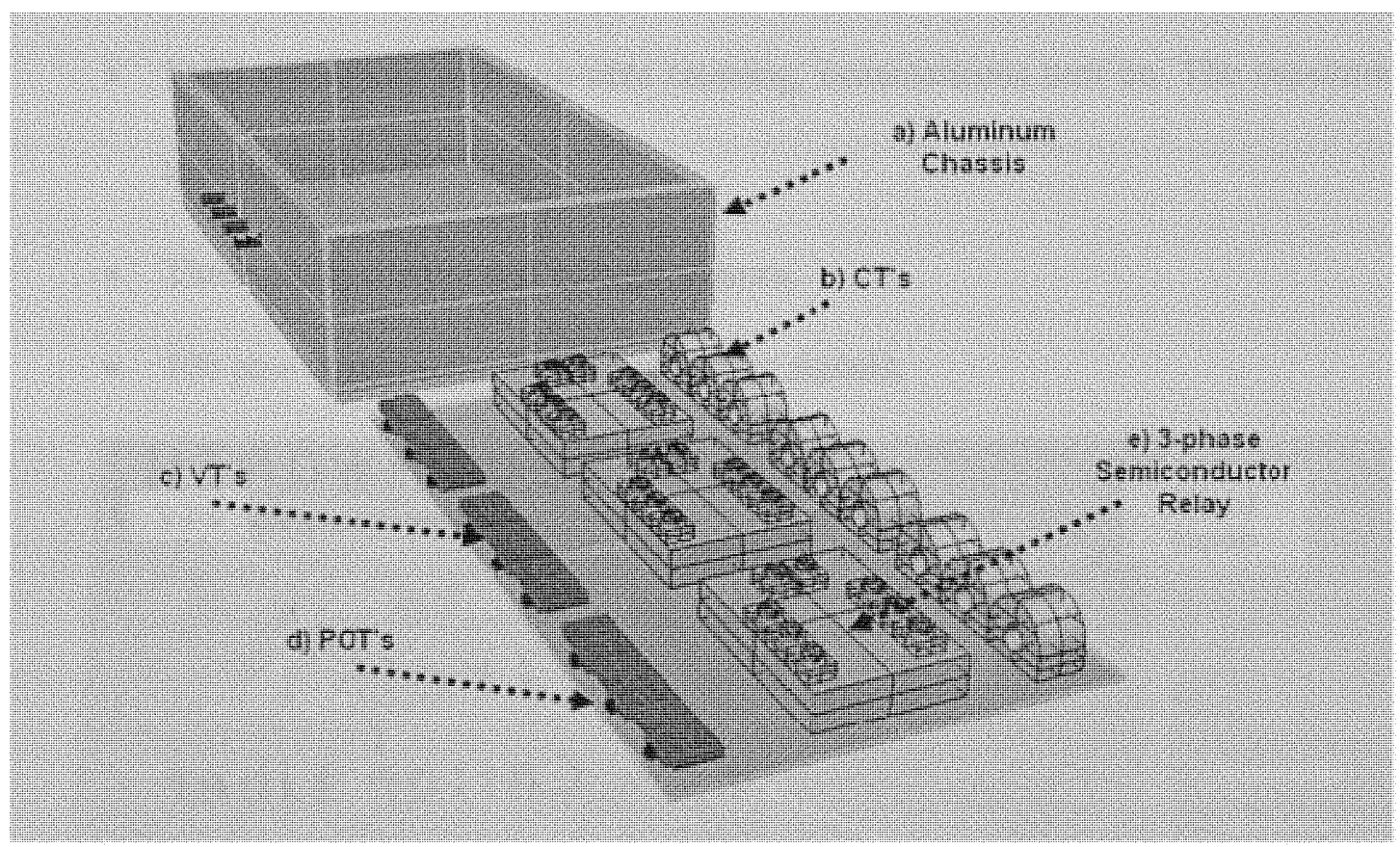

Figure (7.3.4.1) Prototype model for the bus-bar.

The bus-bar is designed to capture 3-phase current profile and 3-phase line to line voltage profile; this is done through the utilization of voltages and current transformers to step-down the voltage to the voltage input level of the analog to digital converter inbuilt in the data acquisition card. 
The developed bus-bar module is presently monitoring the three-phase currents $\left(I_{a}, I_{b}, I_{c}\right)$, three-phase voltages $\left(V_{a}, V_{b}, V_{c}\right)$, frequency, power factor, real power and reactive power.

Figure (7.3.4.2) shows the system bus module. The switching and protection functions were carried out employing the hardware in the loop (HIL) concepts.

The main features of the module are:

- Designed to carry currents up to $20 \mathrm{~A}$ of current and voltages up to $530 \mathrm{~V}$;

- Solid state relay with non-zero crossing, instantaneous turn on. The maximum turn-on time $=1 / 2$ cycle;

- Three phase current and voltage sensors;

- 100 pins terminal for digital and analog signals;

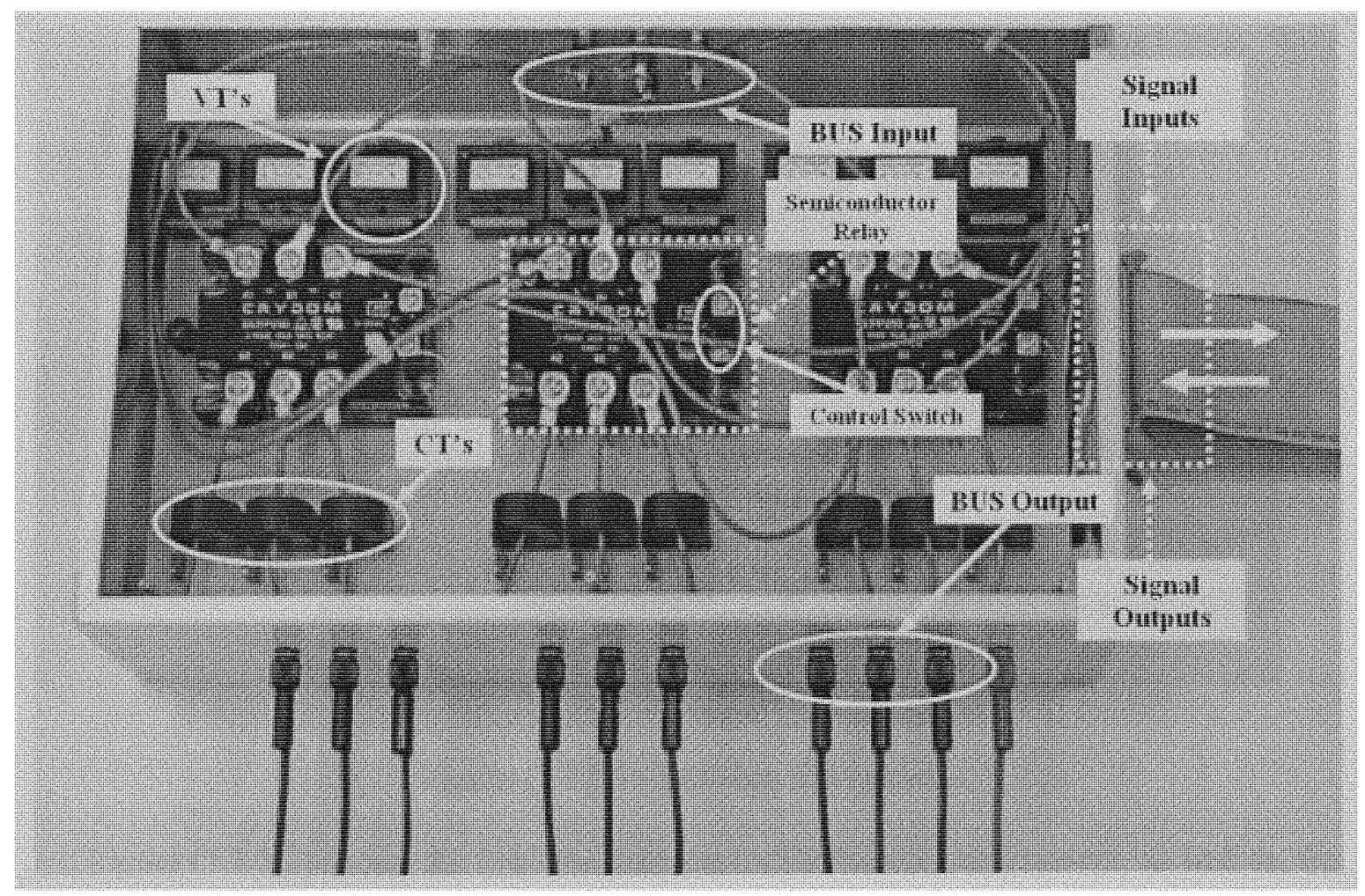

Figure (7.3.4.2) The system bus-bar module. 


\subsubsection{Physical Model of the Overall Test-Bed}

This section presents the integrated hardware test-bed power system developed and utilized to demonstrate the feasibility of the distributed control infrastructure into the power system. Three different test-bed configurations were used for the experimental implementation of distributed control infrastructure. This section describes the three testbed configurations.

The first network configuration is shown in Figure (7.3.5.1) and consists of a two-bus test system with two synchronous generators, and one static load connected at Bus_2 and one TSBR in at Bus_1. The two buses are connected through two parallel transmission line models. This first configuration was used to measure the response of the TSBR model in a simple system with two synchronized generators.

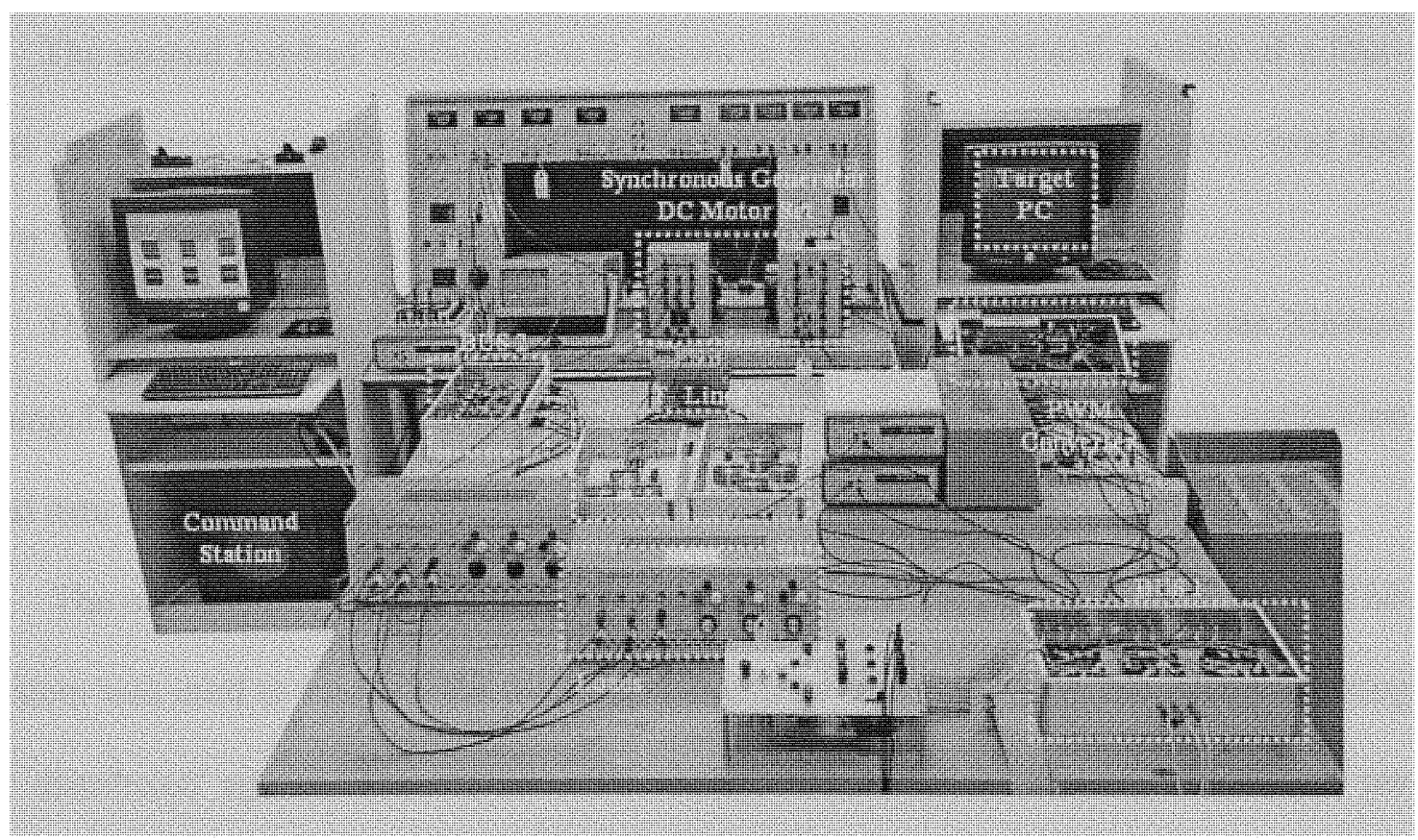

Figure (7.3.5.1) Two-bus test system.

Figure (7.3.5.2) shows the one-line diagram for the two-bus test system; as well as, the RTUs (DAQ system) and control for the TSBR device. 


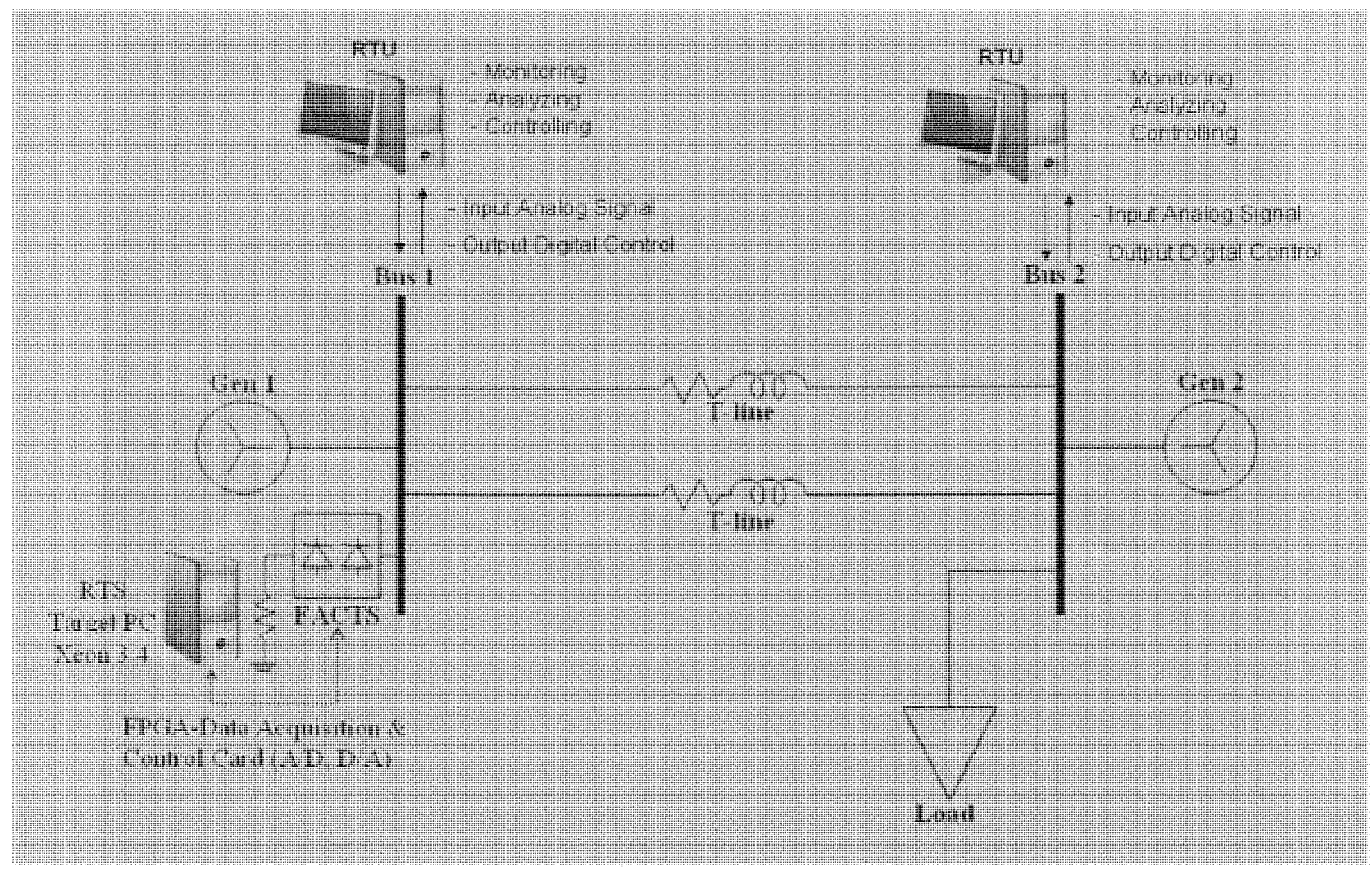

Figure (7.3.5.2) One-line diagram for the two-bus test system.

The second test-bed configuration is shown in Figure (7.3.5.3) and consists of a fourbus test system with two synchronous generators, three static loads, one motor load and two TSBR devices.

This test-bed configuration contains three RTUs, two RTS Agents, and one Mastercomputer station and the necessary telecommunication infrastructure for data acquisition and data sharing.

The one-line diagram for this test-bed configuration is shown in Figure (7.3.5.4). This system was used to evaluate the transient response of the system with two TSBR working as distributed agents. The experimental results and details in the implementation are discussed in the next chapter. 


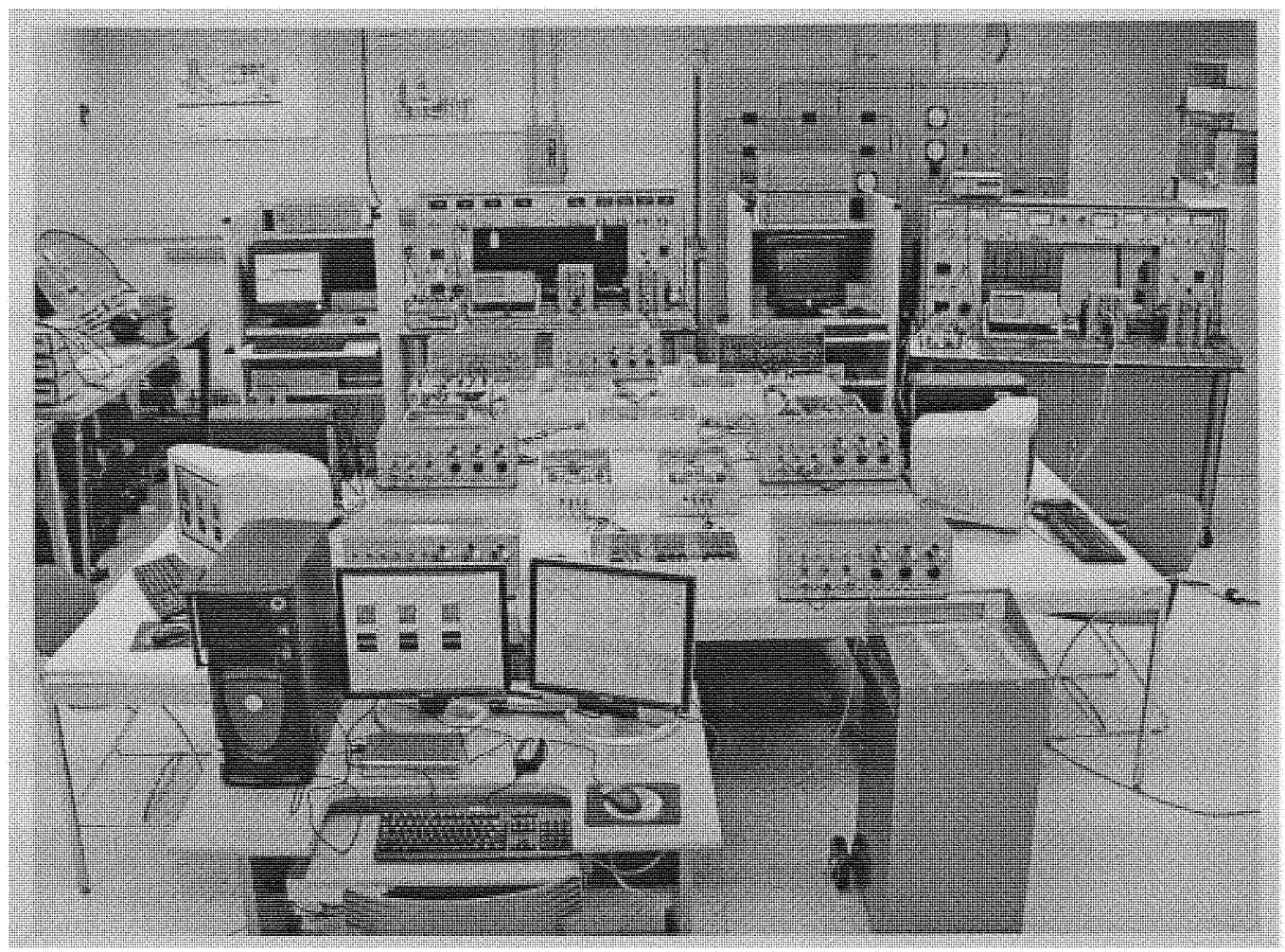

Figure (7.3.5.3) Four-bus test system.

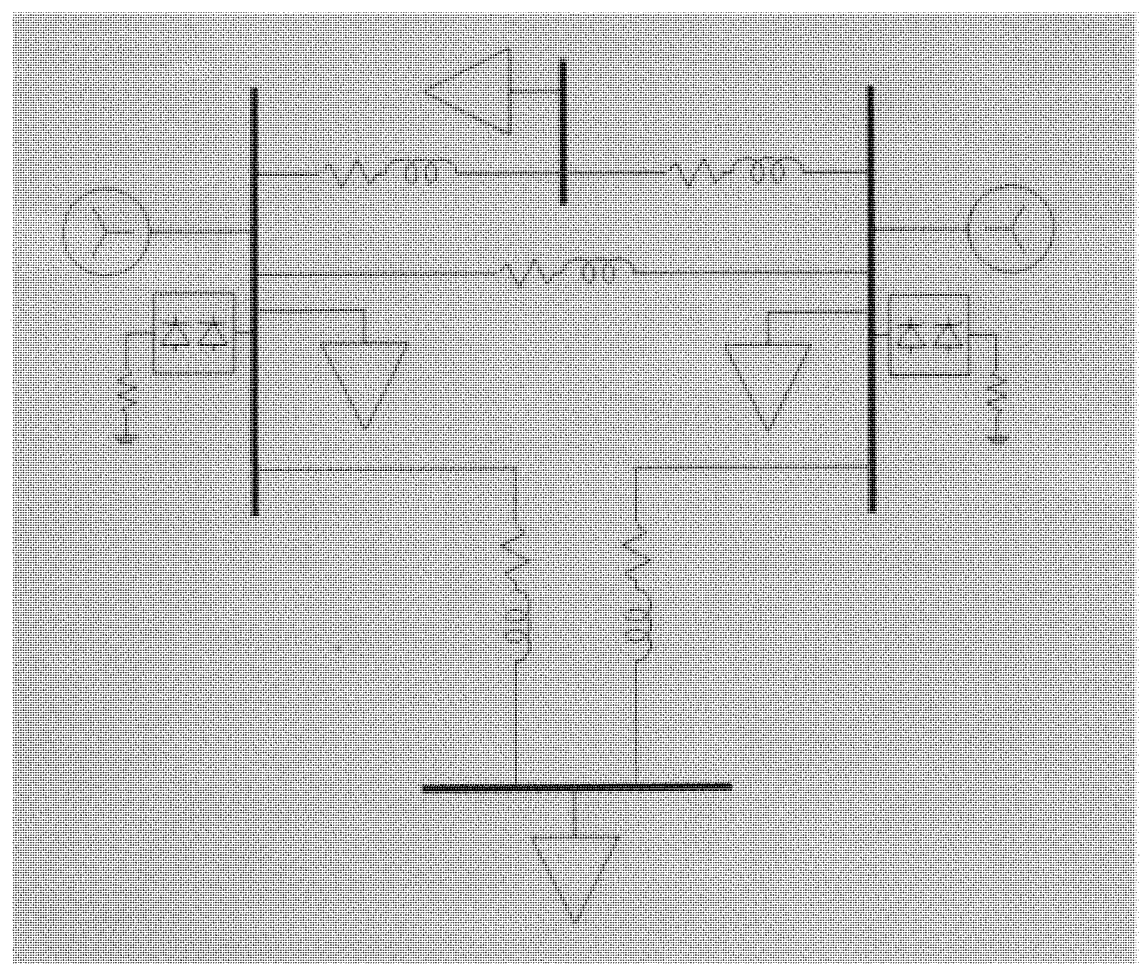

Figure (7.3.5.4) One-line diagram for the four-bus test system. 
The third test-bed configuration shown in Figure (7.3.5.5) is a modified scaled replica of the classical model of a multimachine system; the IEEE standard 9-bus test system. This model is useful for transient stability evaluation in a power system. This system is more representative than the four-bus system, and extensively used for system analysis and control evaluation for transient stability issues.

The 9-bus test system consists of nine buses interconnected by six transmission lines is a ring type interconnection configuration; the system has three generator buses connected to the step-up transformer, respectively. Also, the system includes two TSBR in the distributed control scheme.

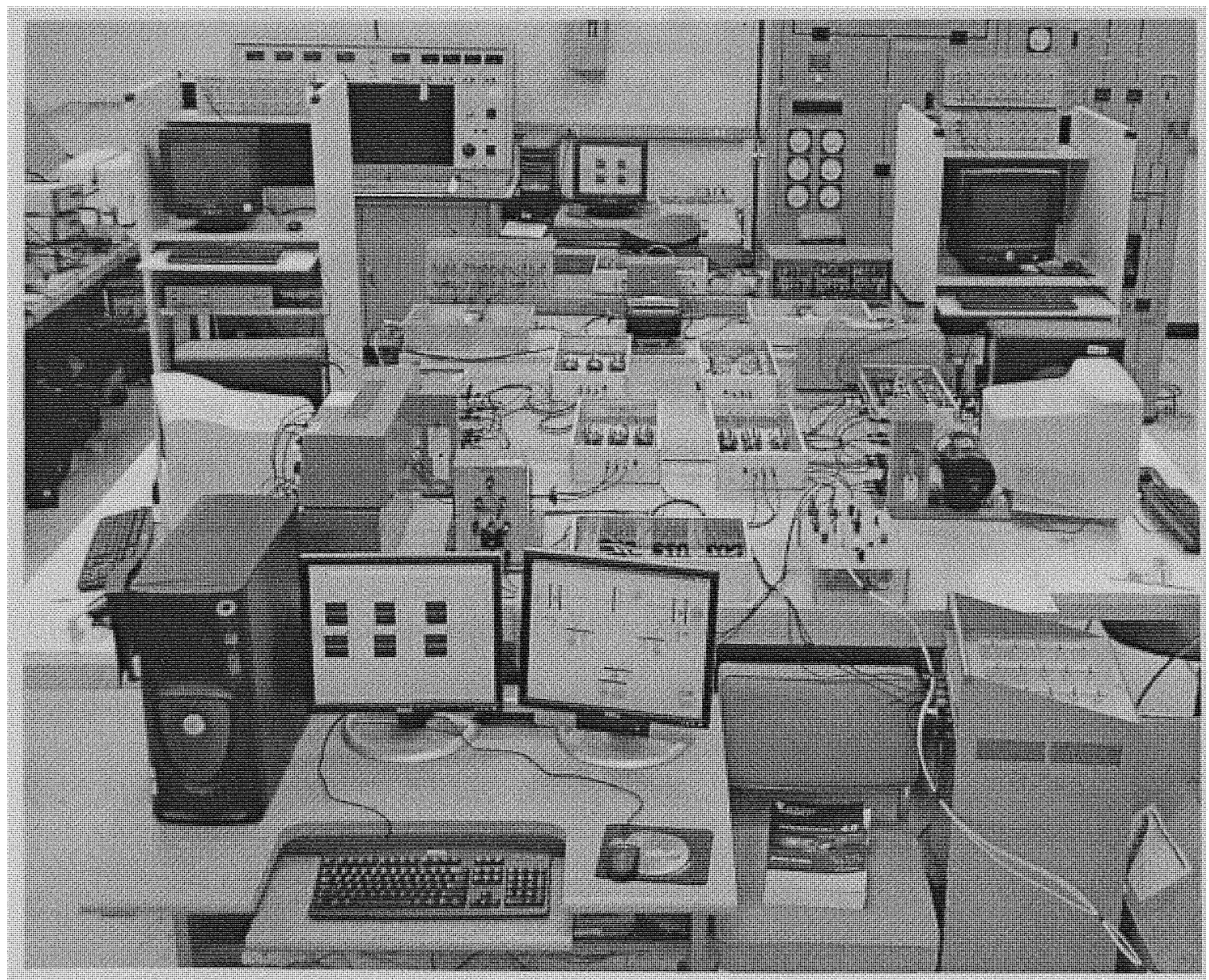

Figures (7.3.5.5) Modify IEEE 9-bus multimachine test system. 
Figure (7.3.5.6) shows the one-line configuration for the 9-bus test system. With the telecommunication network infrastructure included and the distributed control agents.

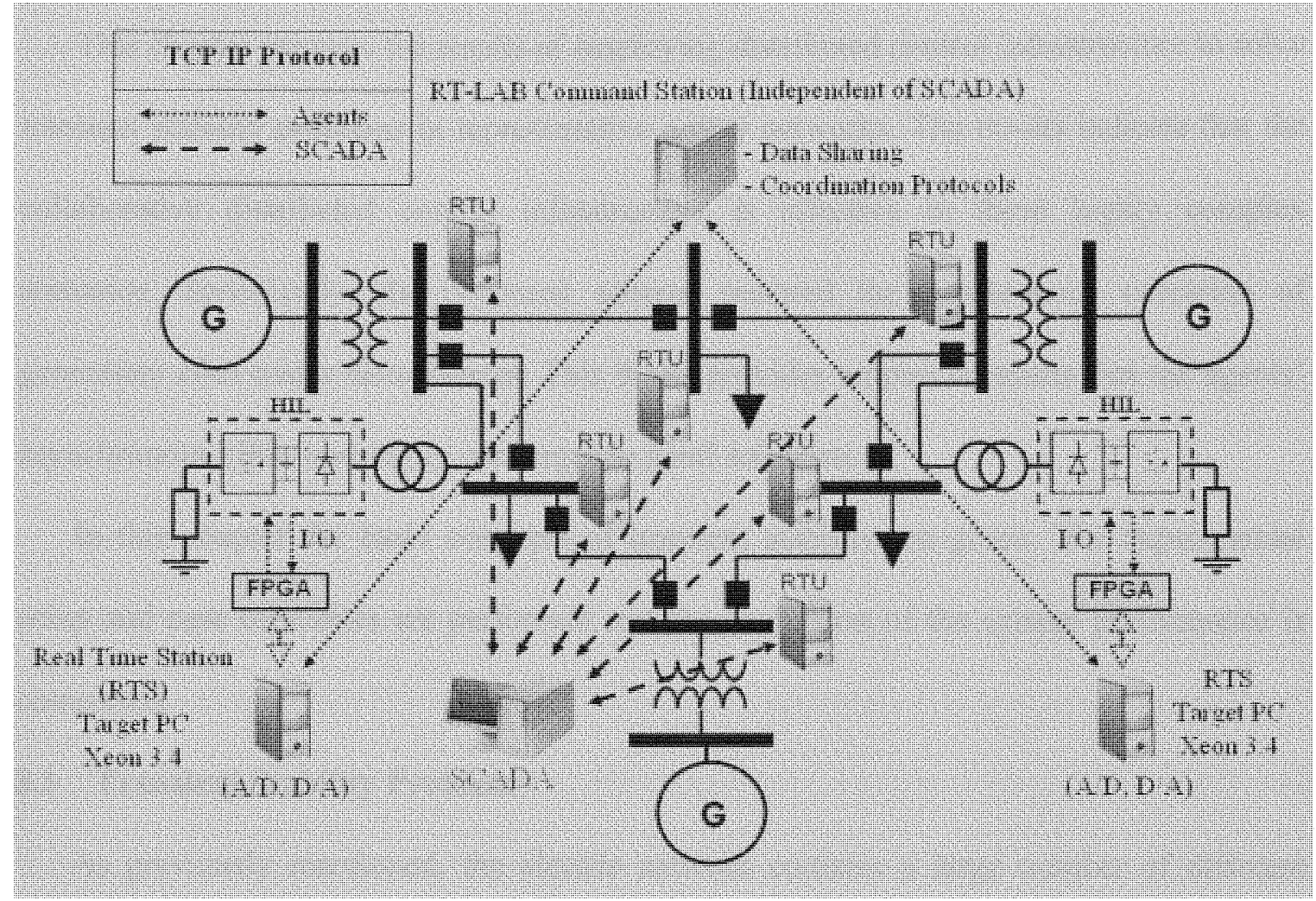

Figure (7.3.5.6) One-line configuration for the 9-bus test system.

As outlined before, the power system simulator requires data sharing to collect data from the RTUs and to coordinate between distributed agents. To address this matter, the laboratory test-bed setup incorporates emerging technologies such as client/server system. Client/server technology, in the context of this thesis, refers to using multiple slave server computers to provide information to a master client computer. 


\section{Chapter 8}

\section{System Implementation and Experimental Results}

This chapter presents the results of employing distributed control infrastructure to solve transient stability problems in representative power networks. The results indicate that the collaborative distributed control infrastructure is effective in these highly complex

scenarios. Specifically, the proposed scheme restored the synchronous operation of generators after major disturbances. This thesis provides empirical results that the agents' asynchronous effort constantly converges to good solutions.

In this chapter the basic concepts of transient stability are given. The experimental setup and case of studies are performed and the evaluation of the system performance is completed.

\subsection{Transient Stability Problem}

As previously mentioned, the electric network contains a large number of different equipments (such as generators, motors, static loads, controllers, and protection devices) and thousands of miles of transmission lines.

Synchronous alternators generate power that is transmitted through the transmission network and delivered to remote sites. When the network is operating under normal conditions, its generating units run at the synchronous frequency and all generators are synchronized. They should remain in this state for safety and technical reasons [20]. In other words if the frequency is higher than the nominal, then there is an excess power and vice versa.

Abnormal conditions, such as the surge of power demand and lightning strikes, create unbalance the mechanical power input and the electric power drawn at the generator. If the 
difference in angle between any two machines increases indefinitely the system will become unstable, the generators will lose synchronization and the system will collapses. The transient stability challenge includes the implementation of preventive and on-line control measures to maintain synchronism between generators. This maintains the adequacy of the supply of power to customers [11]. The physical limitations to accomplish this objective lie in the inability of the control system to quickly adjust the mechanicalpower input to match the electric-power output. This transient stability problem is what we have previously defined as a dynamic control problem (DCP).

As mentioned earlier, FACTS devices open new opportunities for controlling the power flow enhancing the reliability and overall security of power systems. This is possible due to the ability of FACTS to control interrelated parameters that govern the operation of the system including series impedance, shunt impedance, current, voltage, phase angle, and damping of oscillation at various frequencies. These devices can be controlled at high speed and so its influence onto the power system and, thereby, absorb oscillations, and uphold the synchronization between generators.

\subsection{Case of Studies and System Performance Evaluation}

The experimental setup for the different case of studies consists of:

1. Determining the system configuration;

2. Determining the operating constraints;

3. Determining the set of disturbances;

4. Determining the problems that were caused due to the disturbances;

The procedures to evaluate the control strategy for transient stability problems are described in Figure (8.2.1). 


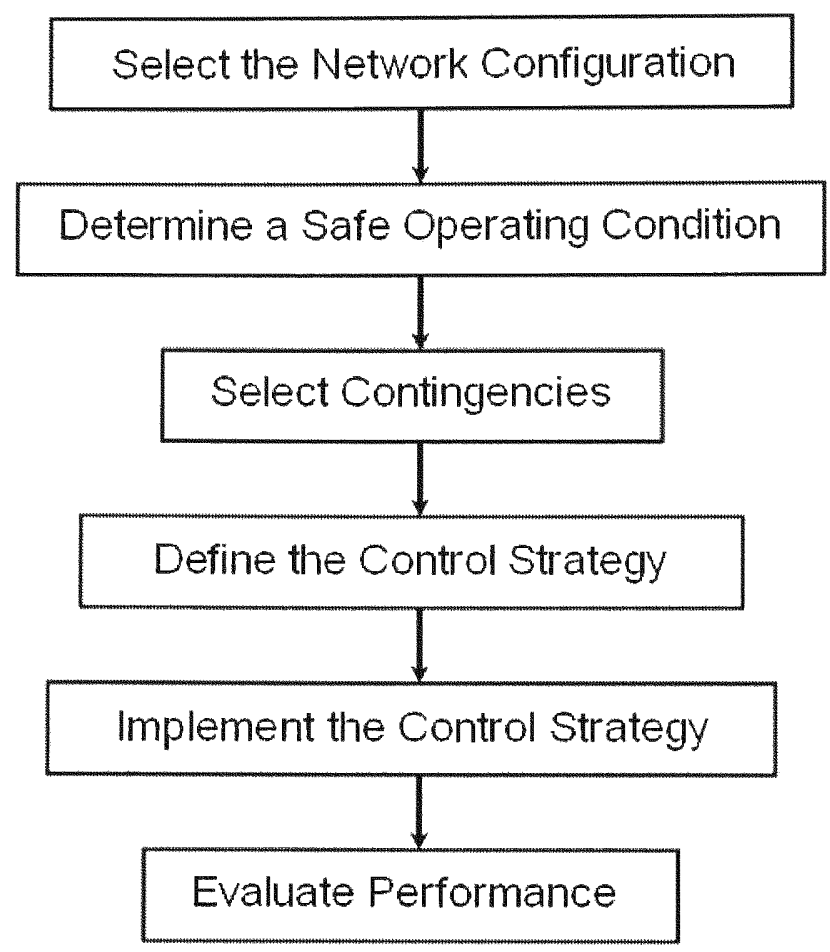

Figure (8.2.1) Procedures to assess transient stability problems.

Four cases studies were used here to evaluate the ability of the distributed control infrastructure to solve the dynamic control problem of transient stability. The four cases are described next.

\subsubsection{Two-Bus Test System Example}

As previously mentioned in chapter 6 , the two-bus test system consists of a two-bus test-bed system with two synchronous generators, and one static load connected at Bus_2 and one TSBR in at Bus_1. The two buses are connected through two parallel transmission lines. This first test system was used to measure the response of the TSBR model in a simple system with two synchronized generators. The system was subjected to a faulty relay that trips one of the lines. The system suffers the imbalance of electrical and mechanical power due to the change in power flow from line_1 to line_2. 
Figure (8.2.1.1) shows the one-line diagram for the two-bus test system, where line_1 is tripped due to the faulty relay.

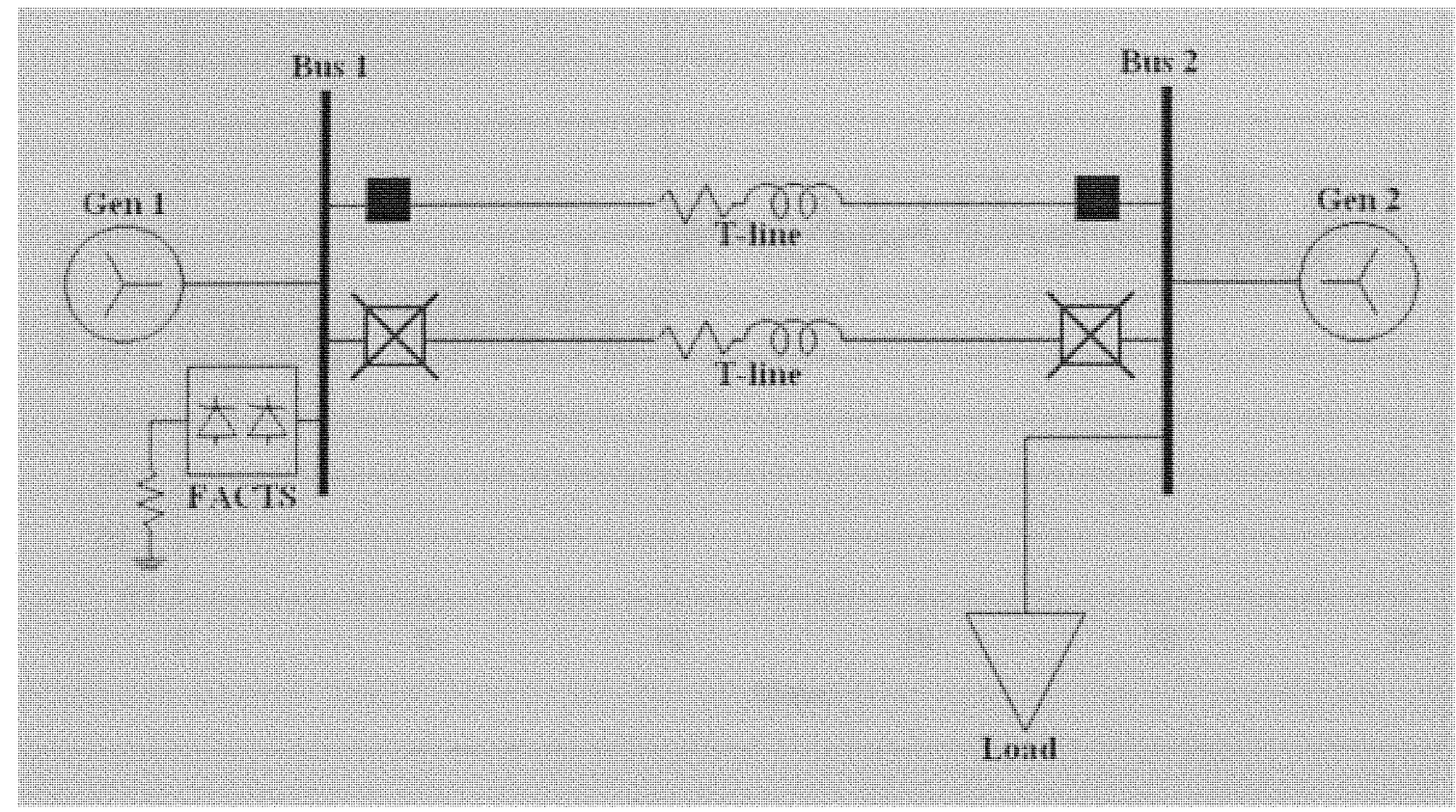

Figure (8.2.1.1) The two-bus test system during the event.

To evaluate the impact of the TSBR in the frequency respond of the system, the system was first assessed without the TSBR and then with TSBR. The result of this event is shown in Figure (8.2.1.2) where the frequency is increased due to the change in the system. The frequency profile shows a slow increase in time, but this could eventually trigger the protective frequency relays and take the system out of synchronism. Another problem that can be observed is the sub-transient oscillation of the system. It is important to notice that this problem is due to the absence of the governor control and the excitation control in the system. 


\section{Withen the TSBR}

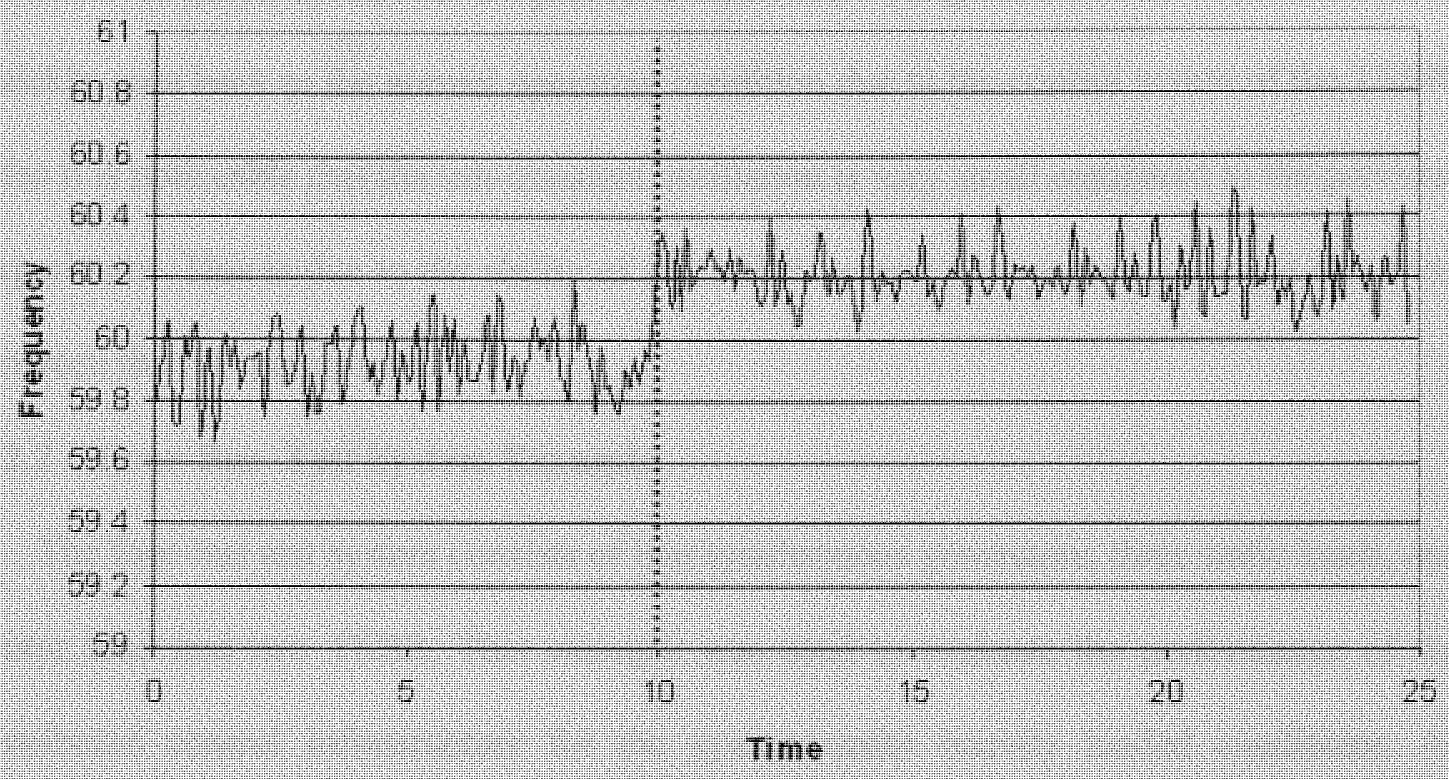

Figure (8.2.1.2) Frequency profile before, during and after the line tripped.

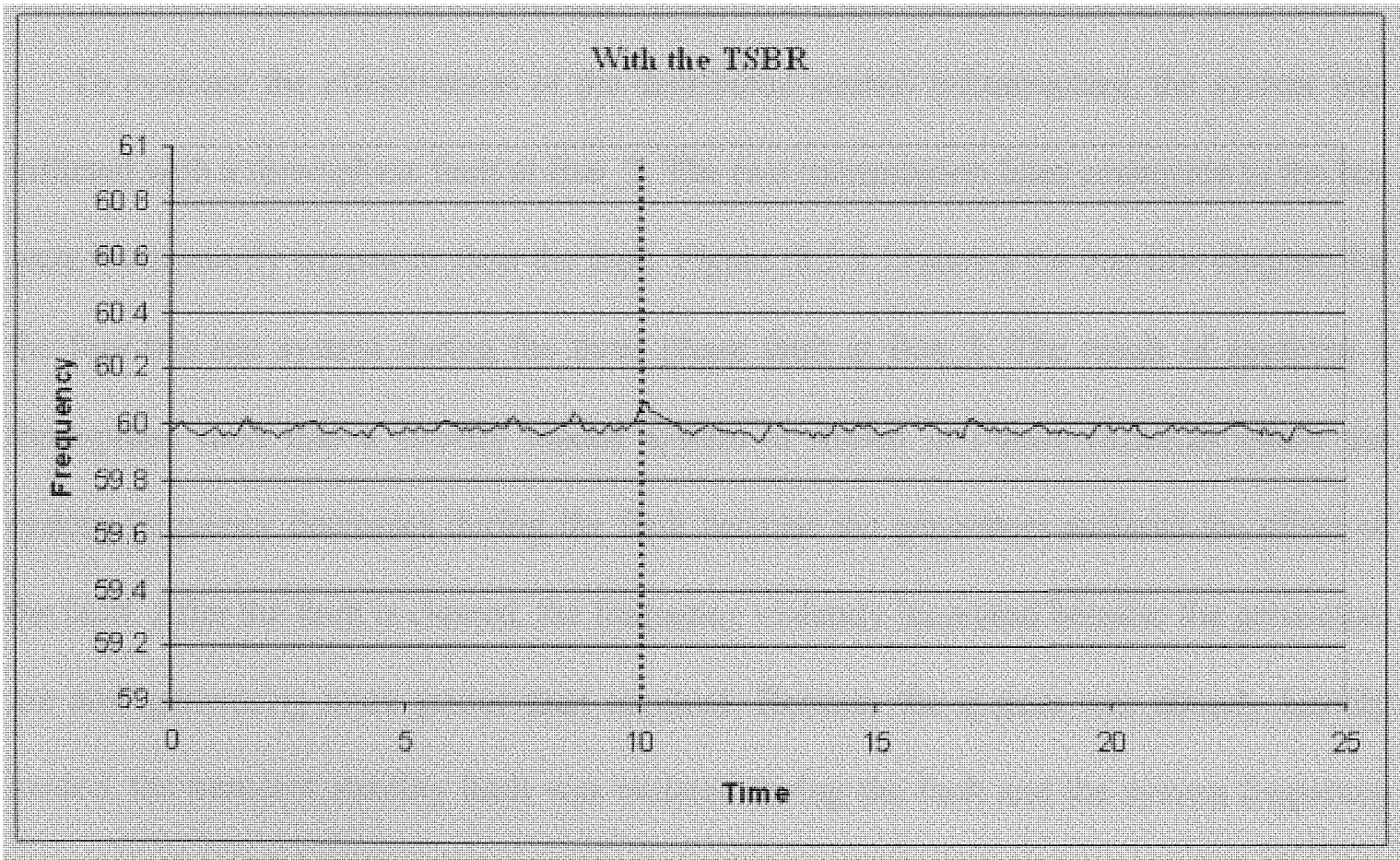

Figure (8.2.1.3) Frequency response of the system with control action. 
Figure (8.2.1.3) shows the frequency response of the system with the addition of the TSBR. It is observed that the frequency is increased during the interval of the event, and quickly goes back to the normal operating condition due to the control action of the TSBR. Also it is noticeable that the sub-transient oscillation is very small compared to the previous experiment. This is due to the ability of the TSBR control to suppress the speed deviation between the two generators.

\subsubsection{Four-Bus Test System Example}

The four-bus test system consists of two synchronous generators, three static loads, one motor load and two TSBR. This example was used to evaluate the performance of the distributed control infrastructure for the system under heavily loaded conditions. As previously stated, the system usually becomes more vulnerable when it is heavily loaded. This also increases the chance of having a cascading event that could end up in a blackout.

To evaluate the effectiveness of the distributed control infrastructure, the system was inflicted with a three-phase fault at Bus_3. The two transmission lines connected to the bus were momentarily tripped to clear the fault. Figure (8.2.2.1) shows the one-line diagram for the four-bus test system during the three phase fault at Bus_3. The result of applying a three-phase fault into a heavily loaded system is shown in Figure (8.2.2.2) where the system frequency decreases during the fault because the generators suffered the effect of the fault. After the fault is cleared, the frequency violently increased because the generators are now generating more power than required. Because the system was already heavily loaded, the transient stability limit was violated during the fault and the system frequency became uncontrollable. In this case, a frequency collapse becomes unavoidable triggering a cascaded event that disconnected the two generators from the system, "BLACKOUT". 


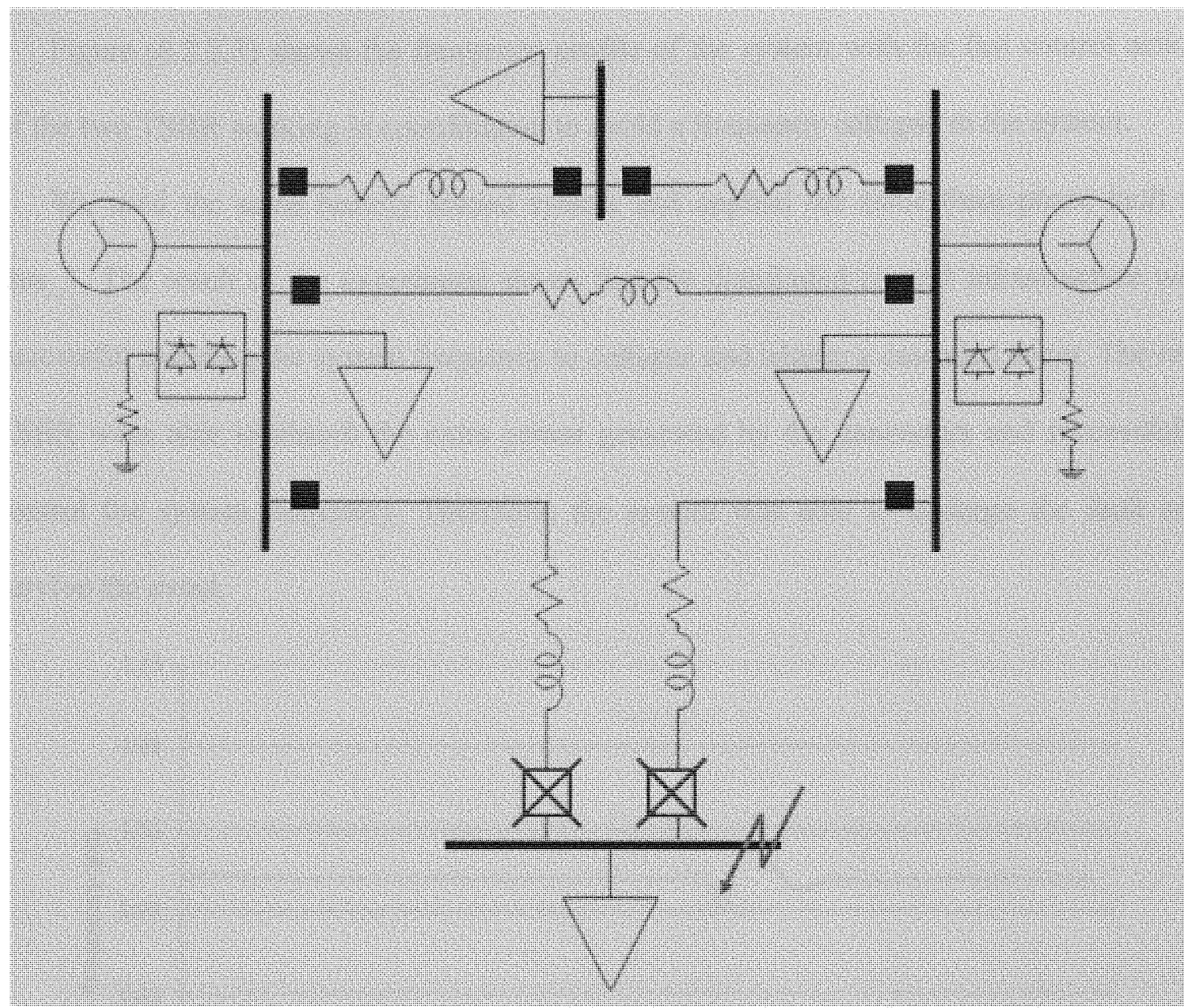

Figure (8.2.2.1) The four-bus test system during the three-phase fault at Bus_3.

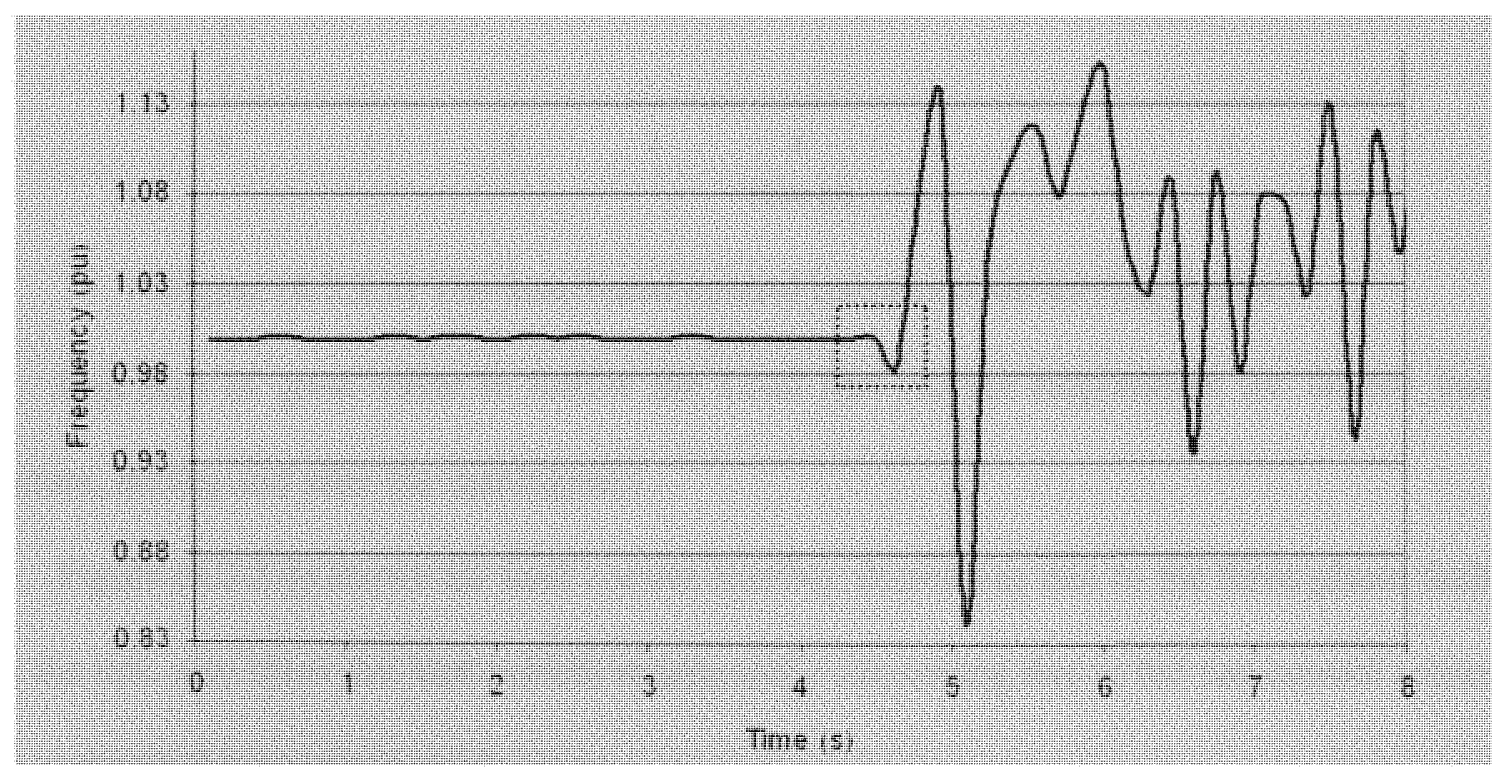

Figure (8.2.2.2) The system frequency collapses and triggered a Blackout. 
Figure (8.2.2.3) shows the frequency profile of the system with the fast control action of the two TSBR working in coordination to avoid a frequency collapse in the system.

We used the same heavily loaded system and applied the same three-phase fault to Bus_3. The result of the experiment shows that the distributed control infrastructure was successful avoiding the system frequency to collapse and trigger a cascaded event. The two TSBR were able to absorb the extra power generated by the generators and successfully control the system frequency. Therefore, the system was able to undergo the fault and survive the event.

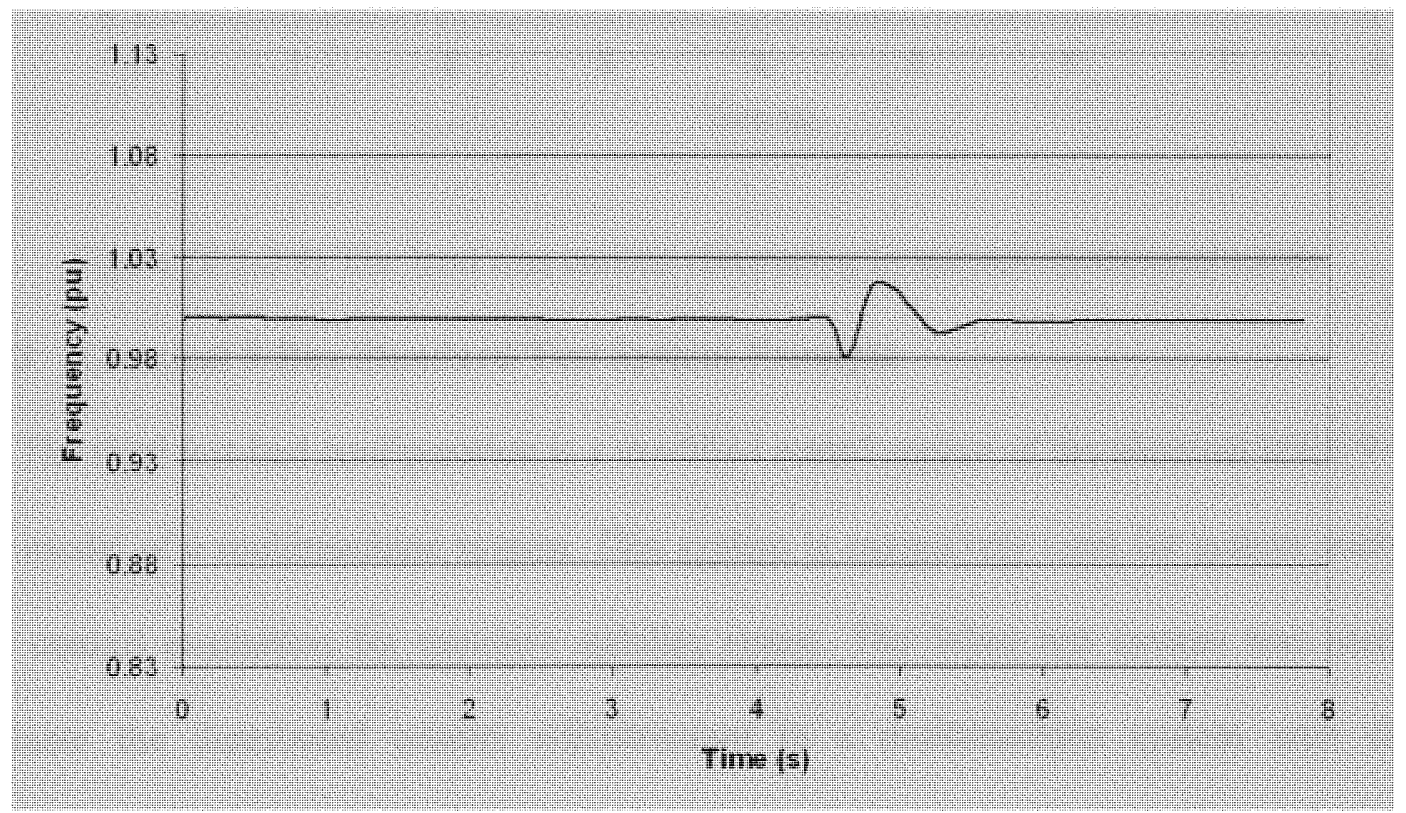

Figure (8.2.2.3) The distributed control infrastructure solving the DCP.

It is also of great value to follow the Load rms current response at the faulted bus before, during and after the contingency.

Figure (8.2.2.4) shows the load current forcibly decreasing due to the three-phase fault at the bus, also show the load current satisfactorily recovering due to the fast control action to maintain the system in stable mode. 


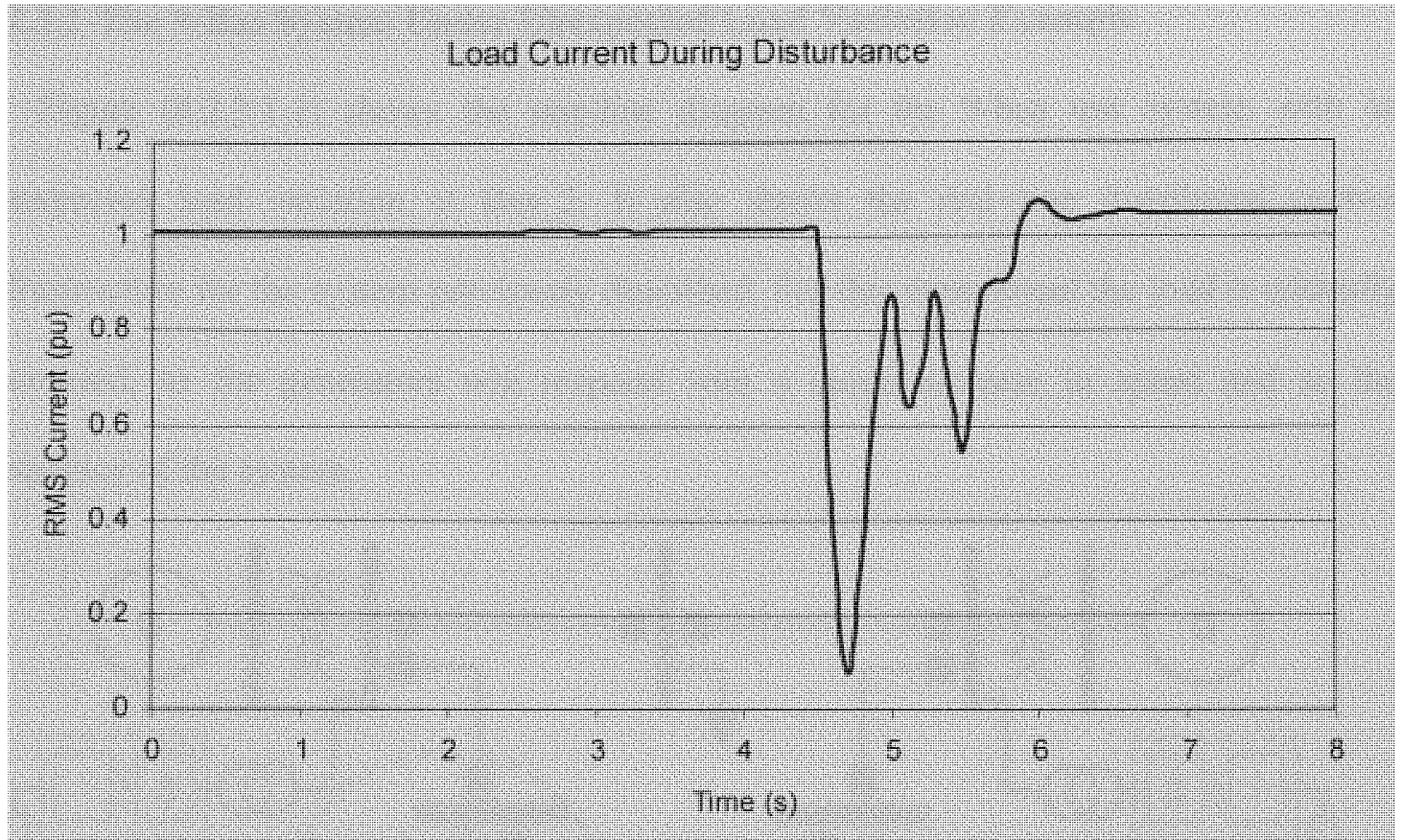

Figure (8.2.2.4) The load rms current recovering to normal operational mode.

\subsubsection{Classical 9-Bus Standard Test System Example}

The third test-bed configuration is a modified scaled replica of the classical IEEE 9-bus test model of a multimachine system. This system is extensively used for system analysis and control evaluation for transient stability issues. The 9-bus test system was used to evaluate the distributed control infrastructure in a multimachine system with three generators and six transmission lines is a ring type interconnection. In order to prove the robustness and the adaptability of the distributed control infrastructure, the system was put to test in two different set of conditions. Theses include:

- Testing the system infrastructure under unexpected load variation.

- Testing the system infrastructure to undergo a fault condition.

The results of these experiments show the flexibility and robustness of the distributed control infrastructure to undergo a fault condition, and react to unexpected events. 


\subsubsection{The 9-Bus System Under Unexpected Load Variation Example}

Figure (8.2.3.1.1) shows the one-line diagram for the 9-bus test system with an unexpected load variation at Bus_2. It also shows the real-time distributed control infrastructure for the system previously described. For this experiment the load is critically decreased, so the generators will respond with a quick increase in speed and so the frequency of the system.

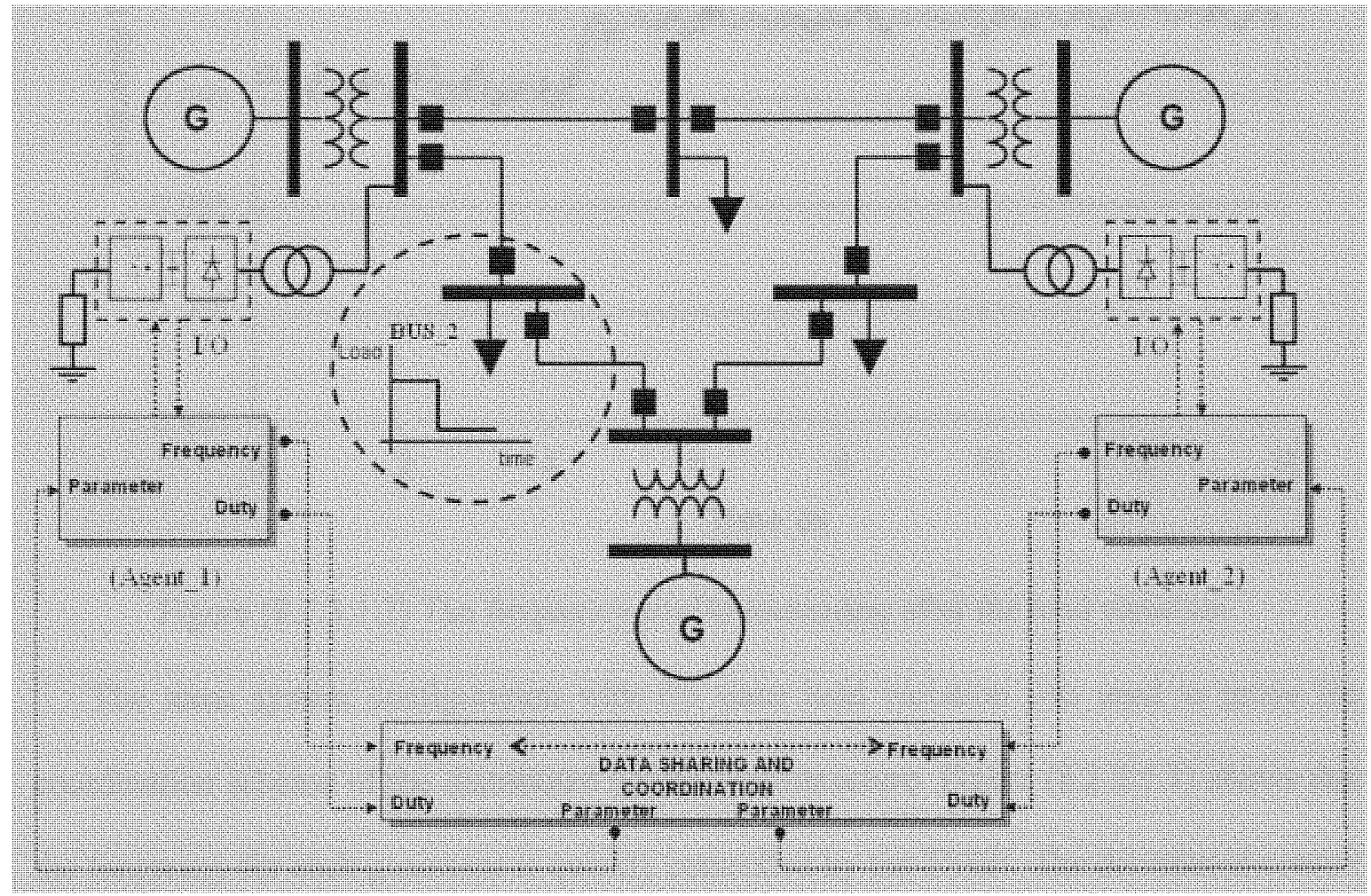

Figure (8.2.3.1.1) The 9-bus test with an unexpected load variation at Bus_2.

The result of critical decrease in the load at Bus_2 is shown in Figure (8.2.3.1.2); this figure shows the system without the implementation of distributed control action. The frequency profile for each generator shows quick increase in the frequency measured at each generating bus and plotted against time. This increase in the system frequency affects 
the power quality delivered to the loads. This also could trigger a cascaded event if it is not controlled.

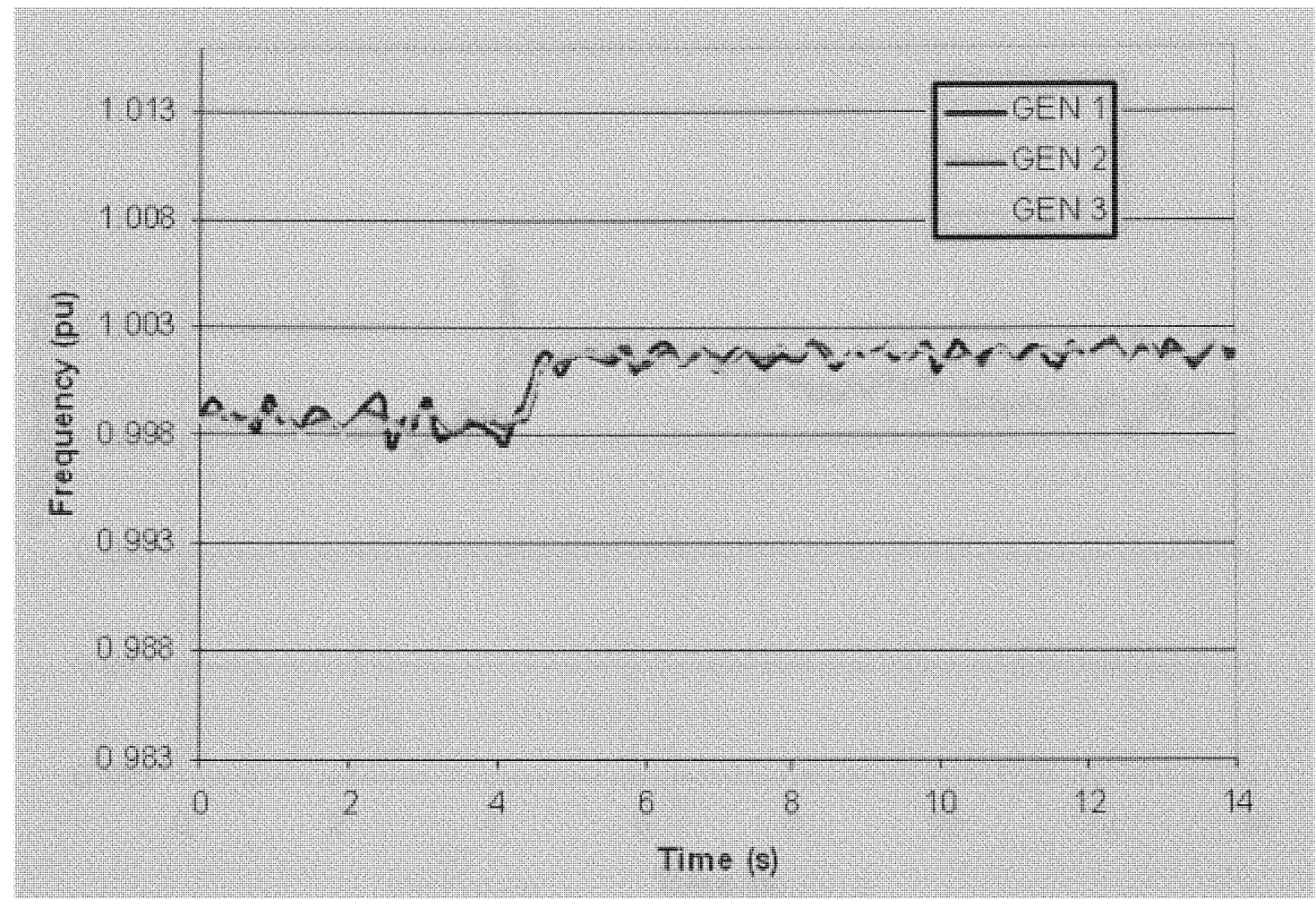

Figure (8.2.3.1.2) The frequency profile increasing after the load variation.

The same system configuration with the same load variation was used to prove the coordination scheme of the distributed control infrastructure. Figure (8.2.3.1.3) shows that the frequency for each generator is quickly controlled by the TSBR. The distributed control infrastructure showed an outstanding performance by braking the three generators without taking them out of synchronism. This complex dynamic control problem was solved by the distributed agents because of its coordination scheme and its parallel operation. The coordination scheme plays a very important part in this type of dynamic control problems. This is because if the agents were not coordinated, then they could take the system out of synchronism by braking one generator more than the other. This is actually the case 
considering that there is no generator control (i.e. governor control, excitation control, automatic voltage regulation (AVR), power system stabilizer (PSS)).

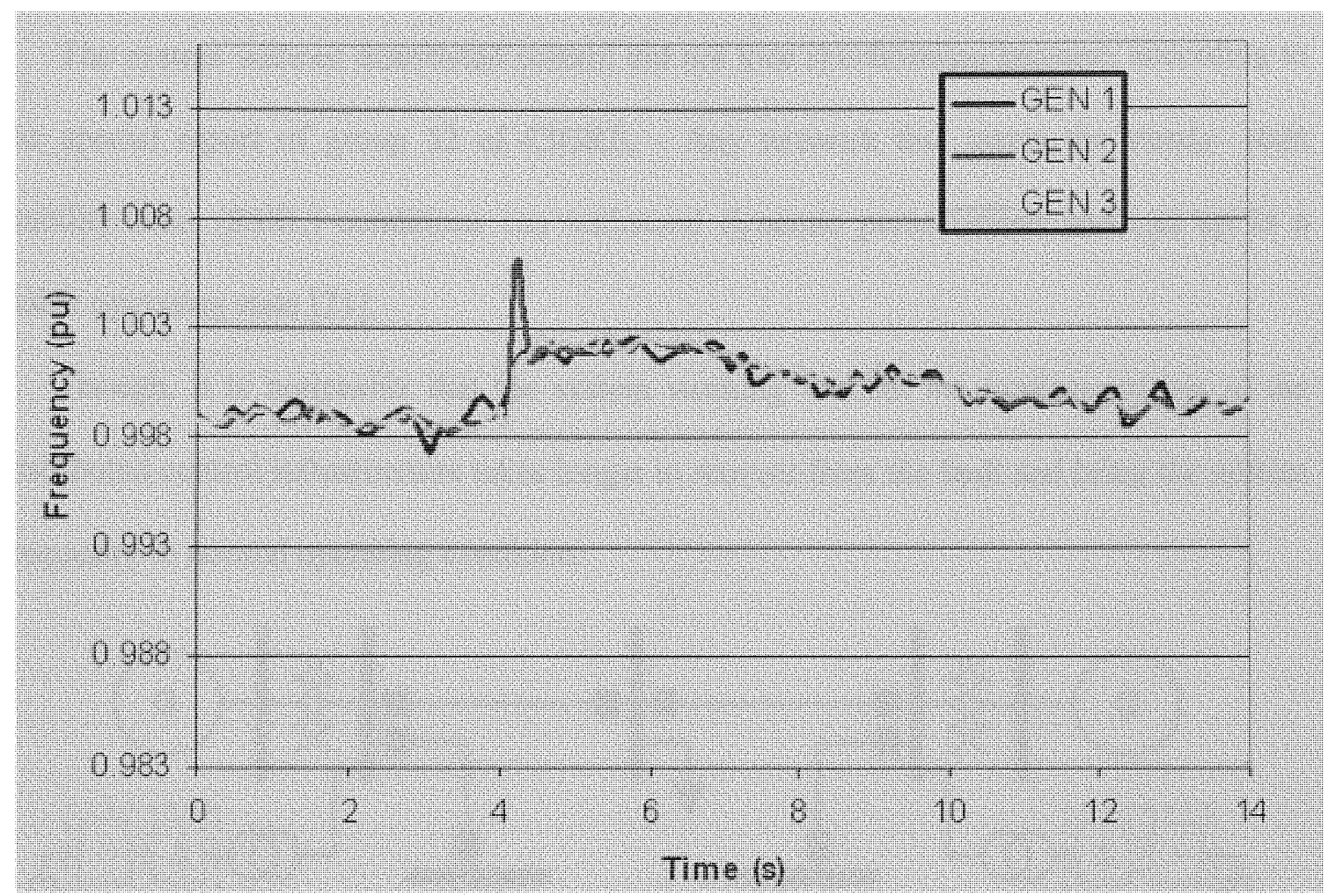

Figure (8.2.3.1.3) The frequency is controlled by the distributed control agents.

\subsubsection{The 9-Bus System Undergoes a Three-Phase Fault Example}

To evaluate the performance of the distributed control infrastructure, the 9-bus system was inflicted with a three-phase fault at Bus_3; the two transmission lines connected to the bus were momentarily tripped to clear the fault. The system frequency decreases during the fault because the generators suffered the effect of the fault. After the fault is cleared, the frequency violently increased because the generators are now generating more power than required.

Figure $(8.2 .3 .2 .1)$ shows the one-line diagram for the 9-bus test system during the three phase fault at Bus_3. The result of applying a three-phase fault into the system is shown in 
Figure (8.2.3.2.2) without the distributed control infrastructure and in Figure (8.2.3.2.3) with distributed control infrastructure implementation.

The system without distributed control did not lose synchronism because, according to the special report of group 32 of CIGRE, - Any network that meets the steady- state stability limit (SSSL) conditions can withstand dynamic perturbations and end up in a stable operating state [21].

Though, it is noticeable that the system working without the implementation of the distributed control infrastructure takes longer to recover from the fault and bring the system frequency to normal operating condition.

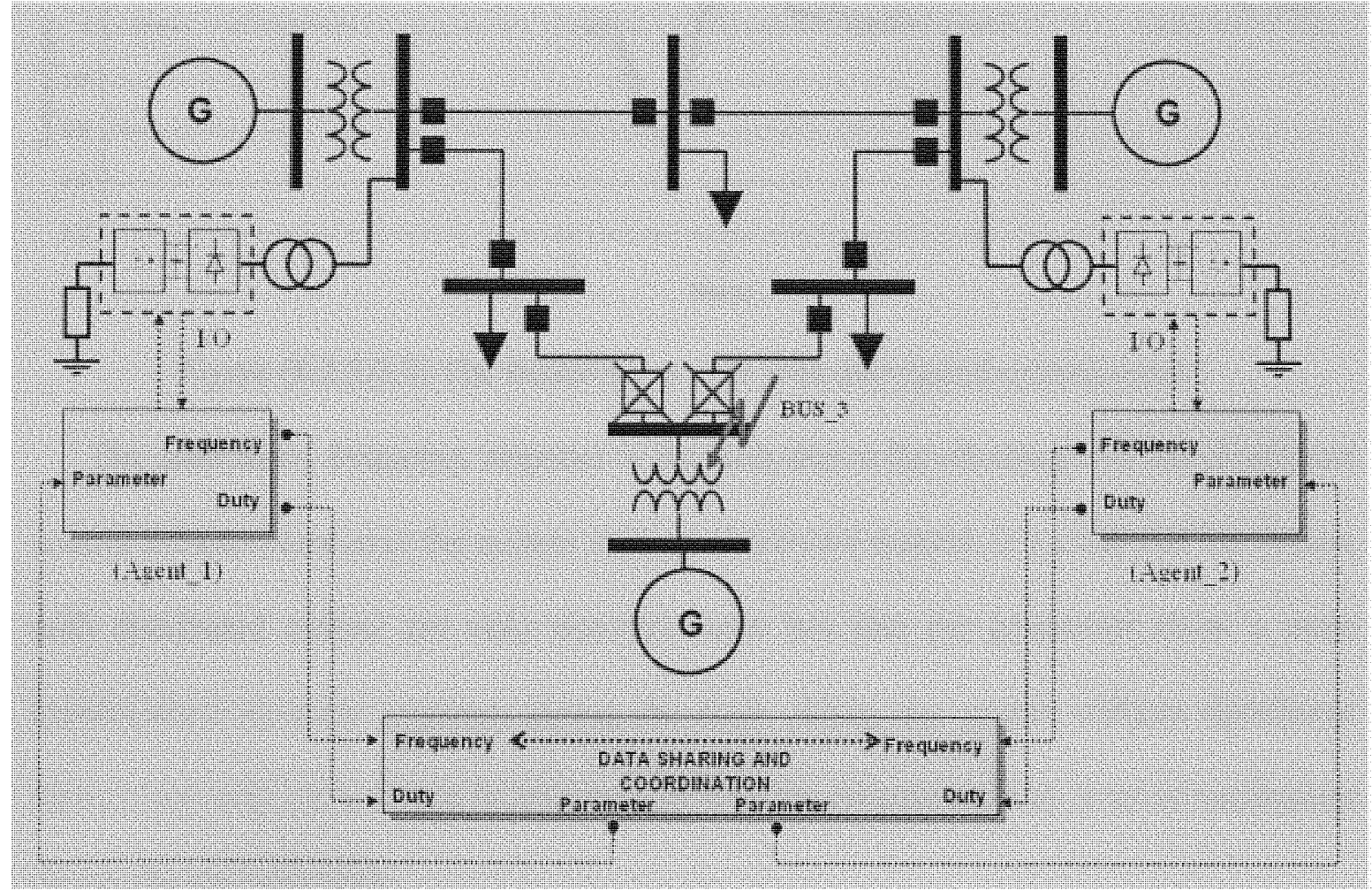

Figure (8.2.3.2.1) the 9-bus one-line diagram during the three phase fault at Bus_3. 


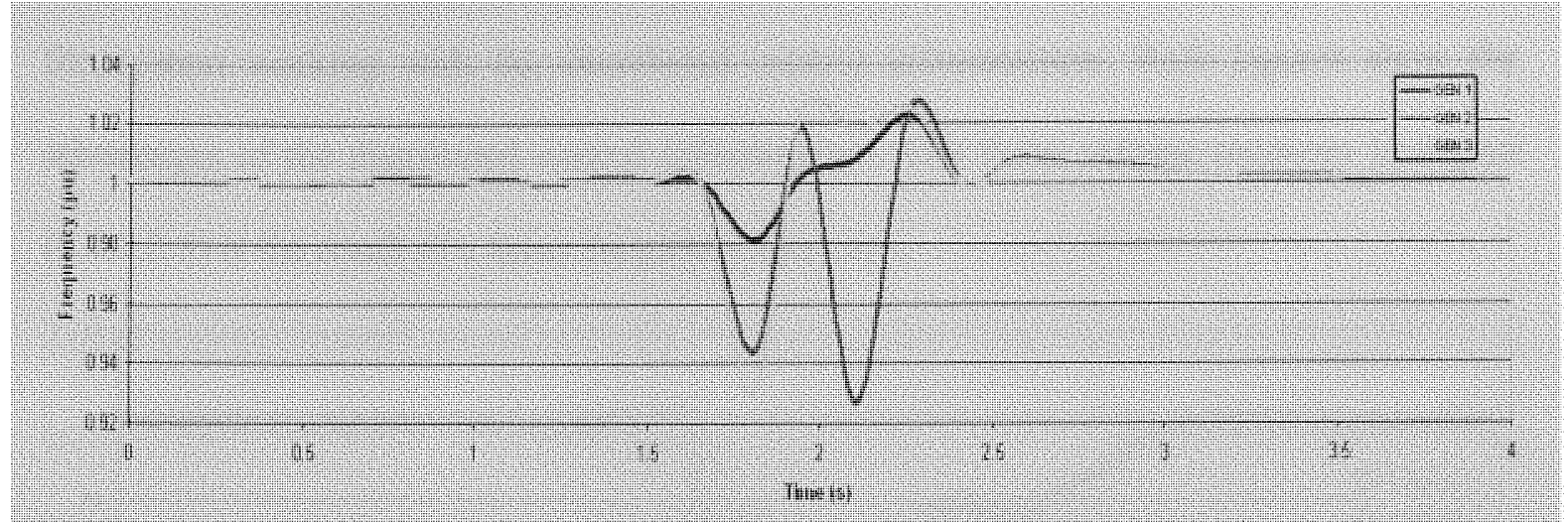

Figure (8.2.3.2.2) The frequency profile without the distributed control agents.

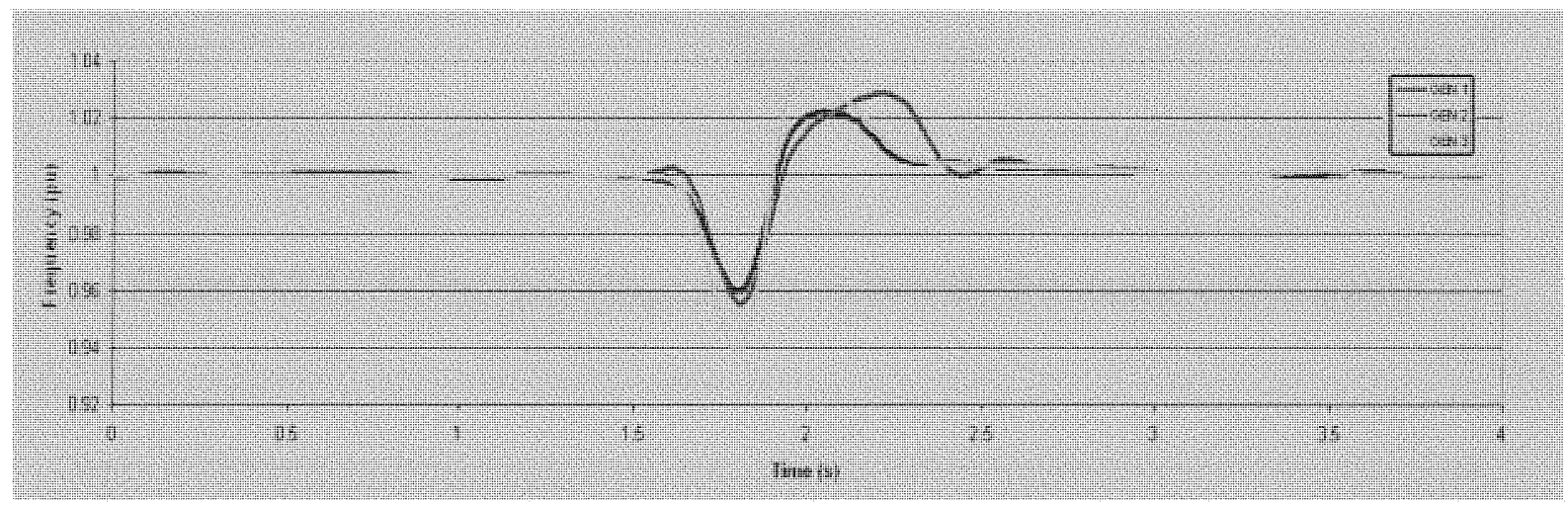

Figure (8.2.3.2.3) The frequency profile with distributed control agents.

The generators' frequency response to the fault shows the advantages of the distributed control infrastructure over the system without distributed control. It is clear that the system with distributed control not only recovers faster but also the generators show a more synchronized dynamic response during the fault. While the system without distributed control, not only takes longer to recovers but also the generators show a lot of oscillations during the recovery time.

For this experiment, the rms voltage profile for the nine buses were recorded and compared. Figure $(8.2 .3 .2 .4)$ shows the rms voltage profile for the system working without the distributed control infrastructure. Figure (8.2.3.2.5) shows the rms voltage profile for 
the system working with the distributed control infrastructure. Both figures show the advantages of the distributed control infrastructure. The faster voltage recovery in Figure $(8.2 .3 .2 .5)$ is the result of a faster frequency recovery.

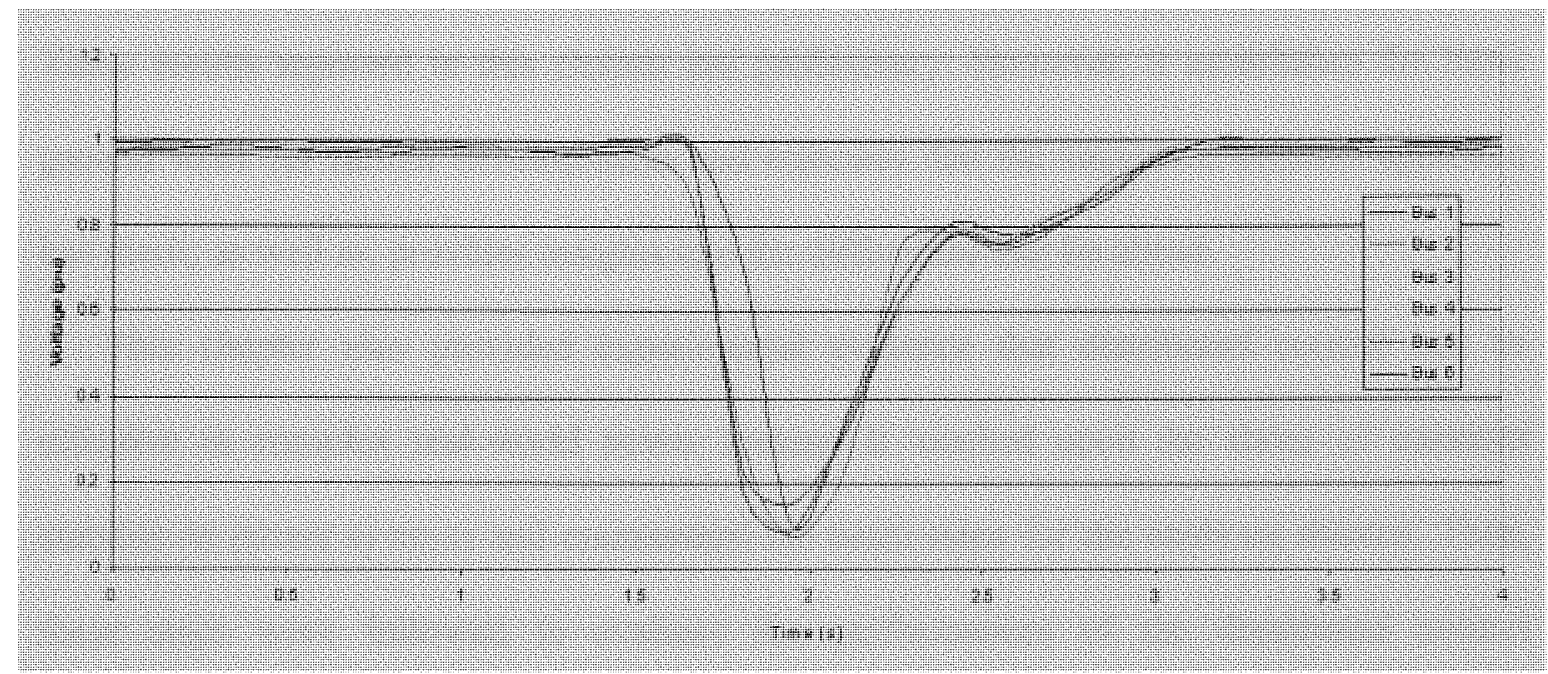

Figure (8.2.3.2.4) The rms voltage profile without the distributed control action.

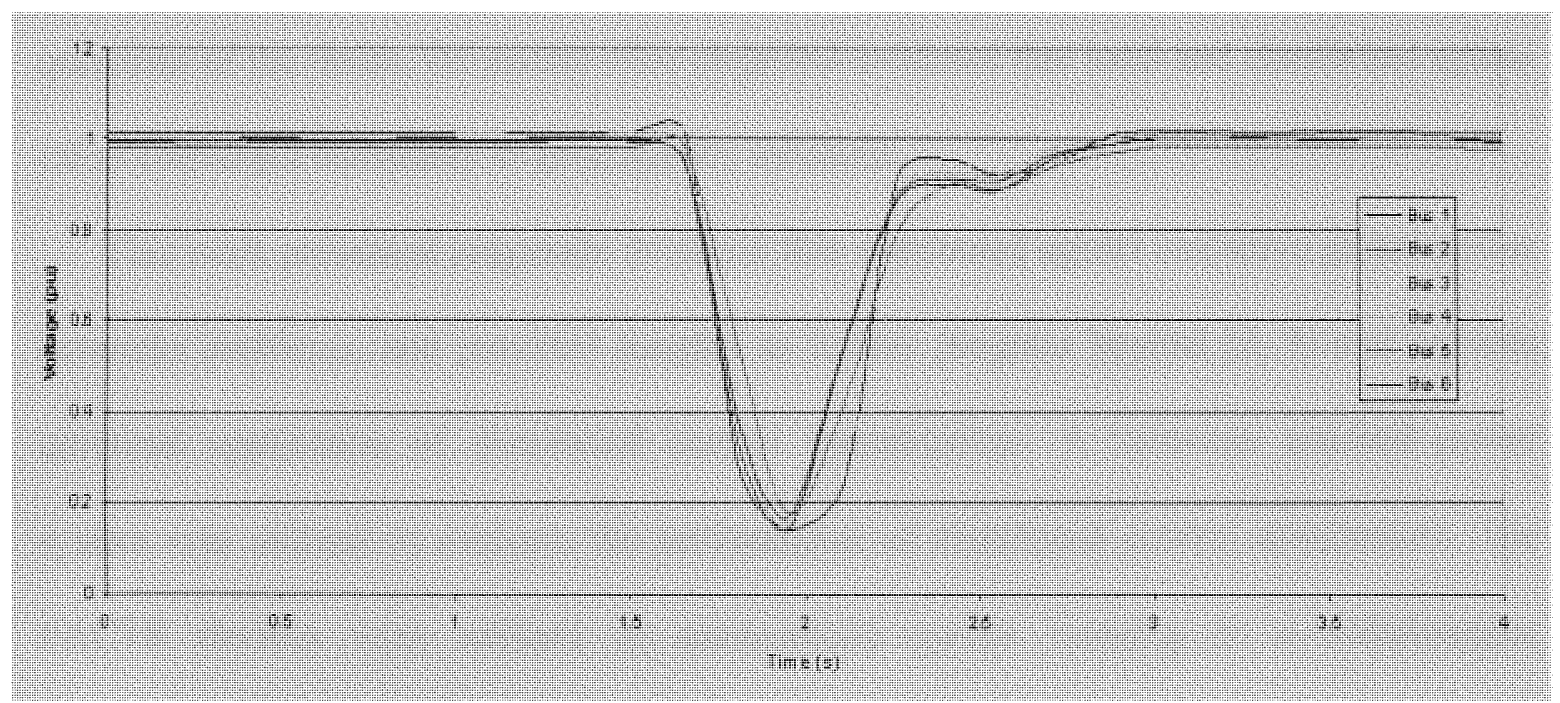

Figure (8.2.3.2.5) The rms voltage profile with the distributed control action. 
These case studies show the different disturbances and their effect on the system's frequency. Also, the system was tested for normal and abnormal system variations, such as normal load variations and abrupt variation either in generation or loads.

These results reported in this chapter are preliminary. However, it establishes the basic ground work for further developments and indicates that the suggested solution approach has merit; as well as, provides insights into the difficulties that lie ahead.

This chapter also provided experimental evidence that distributed control can be effective in complex real-world power system. It touched upon the status of transient stability in power systems and enumerated the basic steps for the analyses thereof. Then, these steps were followed to evaluate the performances of the distributed control infrastructure in three test power systems. This included the two-bus test-bed system, the four-bus test-bed system and the standard 9-bus network. Finally, with respect to the core questions of the thesis, the results offer empirical evidence that local and distributed agents effort converges constantly to good quality solutions.

This work presented pragmatic evidence that distributed agents can solve global power networks problems, and provided insights as further progress beyond $[5,8]$ and towards universal application of distributed control infrastructure. A hardware test-bed power system was utilized to evaluate the performance of distributed control architecture. The FACTS devices were utilized as autonomous agents working in a collaborative telecommunication network infrastructure.

Next we discuss the results of the experiments and provide recommendation for future work. 


\section{Chapter 9}

\section{Discussion and Results}

As previously stated, cascading failures and blackouts result from violations that have been allowed to persist long enough to trigger the protective system.

The specific goal of this work was to develop a test-bed system to prove that a distributed network infrastructure with autonomous agents is capable of eliminating power system violations before the protection system acts to disconnect equipment.

This means that agents should locally suppress violations while maintaining everything else constant, then the method is capable of increasing the overall reliability of the system without conflicting with other objectives (i.e. economics, reliability, protection).

Experiments performed for this thesis demonstrate that:

- The applicability of the developed test-bed to conduct system stability studies and to evaluate the strategies of distributed control infrastructure.

- That the utilization of FACTS devices allowed direct and constant control on the parameter that govern the performance of power system; this open opportunities to effectively utilize FACTS as local independent agents working in a collaborative network.

- It is possible to design a distributed control infrastructure of autonomous agents with limited communication abilities that can eliminate power network violations before they can trigger the protective system.

These are important results; because it implies that it is possible to solve some global power network problems without centralized control infrastructures. Finally it is believed that it is possible to develop a self-monitoring, self-corrective and self-healing power grid. 


\section{Chapter 10}

\section{Recommendation and Future Work}

This chapter addresses the recommendation for future work. With additional development, it seems probable that this technology could improve the ability of power systems to make better tradeoffs between conflicting objectives. In future work, it is recommended to refine this method, perhaps through the use of improved network models, and further study the benefits, costs, and risks of distributed agents for power networks.

The coordination and cooperation protocols and rules could be greatly improved through the implementation of intelligent operation such as Artificial Neural Networks, Expert Systems, Fuzzy Logic, and Genetic Algorithms. This would increase the adaptability of independent agents.

Also TSBR is working only in the braking mode in this thesis. It is the major importance that the system could also operate in an energy supply mode; instead of just wasting the extra energy of the generator while a speed deviation is created. The TSBR could be modified to store the extra energy for later injection back to the system.

The utilization of different type of FACTS devices is also encouraged; UPFC and STATCOM could have a much better impact as autonomous distributed agents.

The test-bed system can be further improved by creating a generator control system, (i.e. excitation control, governor control).

The test-bed software subsystem could also be improved by adding an on-line load flow program in the master computer; as well as, on-line alarms for voltage and loading violations, a program to determine the available transfer capability. The system should also include all the protection algorithms to perform protection and coordination analysis. 


\section{REFERENCES}

[1] I. Dobson, B. A. Carreras, D. E. Newman, "A criticality approach to monitoring cascading failure risk and failure propagation in transmission systems", Proc. of the Carnegie Mellon Transmission Conf., Pittsburgh, 2004.

[2] G. S. Vassell, "Northeast Blackout of 1965", IEEE Power Engineering Review, January, 1991.

[3] NERC Disturbance Analysis Working Group, "Western Interconnection (WSCC) System Disturbance - August 10, 1996", NERC 1996 System Disturbances Report, Aug 2002, Available: http:// www.nerc.com

[4] O. A. Mohammed, N. Cristaldo, N. Abed, "Secure Power Grid Infrastructure Simulation", Power Engineering Society, Tampa, June 2007.

[5] S.N. Talukdar and E. Camponogara, "Collaborative Nets," Proceedings of the $33^{\text {rd }}$ Hawaii International Conference on System Sciences, January 2000.

[6] E. Camponogara, "Controlling Networks with Collaborative Nets", Doctoral Dissertation, Carnegie Mellon University, Pittsburgh, Pennsylvania, September 2000.

[7] C. Rehtanz, "Autonomous Systems and Intelligent Agents in Power System Control and Operation", Springer, 2003.

[8] C. Rehtanz, E. Handschin, C. Becker, "Coordinated Decentralize Control of FACTS Devices Applying Autonomous System", International Conference of Electrical Engineering, ICEE, Kyongju, Korea, 1998.

[9] P. W. Sauer, M. A. Pai, "Power System Steady State Stability and the Load Flow Jacobian", IEEE Transaction on Power Systems, November, 1990.

[10] R. H. Miller, "Power System Operation", McGraw-Hill Inc., 1983.

[11] P. Kundur, "Power System Stability and Control", McGraw-Hill Inc., 1994.

[12] N. Mohan, T. M. Undeland and W. P. Robbins, "Power Electronics - Converters, Applications, and Design", John Wiley and Sons Inc, 2003.

[13] N. G. Hingorani and L. Gyugyi, "Understanding FACTS", IEEE Press 2000.

[14] P. M. Anderson and A. A. Fouad, "Power System Control and Stability", Iowa State University Press, 1977. 
[15] O. A. Mohammed, N. Y. Abed, and S.C. Ganu, "Real-Time Simulations of Electrical Machine Drives with Hardware-in-the-Loop", IEEE/PES Meeting, 2007.

[16] Hampden Engineering Corporation, "Bulleting 252G - Model HMD 100 CM", East Longmeadow, MA, 2007.

[17] Opal-RT, "RT-Lab User Manual", 2004.

[18] H. Saadat, "Power System Analysis", McGraw-Hill Inc., 1999.

[19] IEEE Task Force on Term \& Definitions, "Proposed Terms \& Definitions for Power System Stability", IEEE Transactions on Power Apparatus and Systems, July 1982.

[20] M. Magnien, "Rapport special du Grupe 32 Conception et Fonctionnement de Reseaux", Conference Internationale des Grands Reseaux Electriques a Haute Tension, CIGRE Session, 1964.

[21] S. C. Savulescu, "A Metric for Qualifying the Risk of Blackout", Real Time Stability Challenge Panel Session, Power Systems Conference and Exposition, New York, 2004 .

[22] NERC, "Available Transfer Capability Definitions and Determination", North America Reliability Council, June, 1996. 


\section{Power System Stability Assessment}

Any network that meets the steady state stability limit (SSSL) conditions can withstand dynamic perturbations and end up in a stable operating state [20]. This means that SSSL can be expressed for a given topology and set of voltages conditions, the SSSL is the total MW loading of the system. This also means that SSSL and TSL are closely related, although until now there is no mathematical model that shows such relationship [21]. Once the stability limits are set for the scheduled system's loading; the TTC and TRM can be easily calculated and locally monitored in real-time as shown in Figure (A.1)

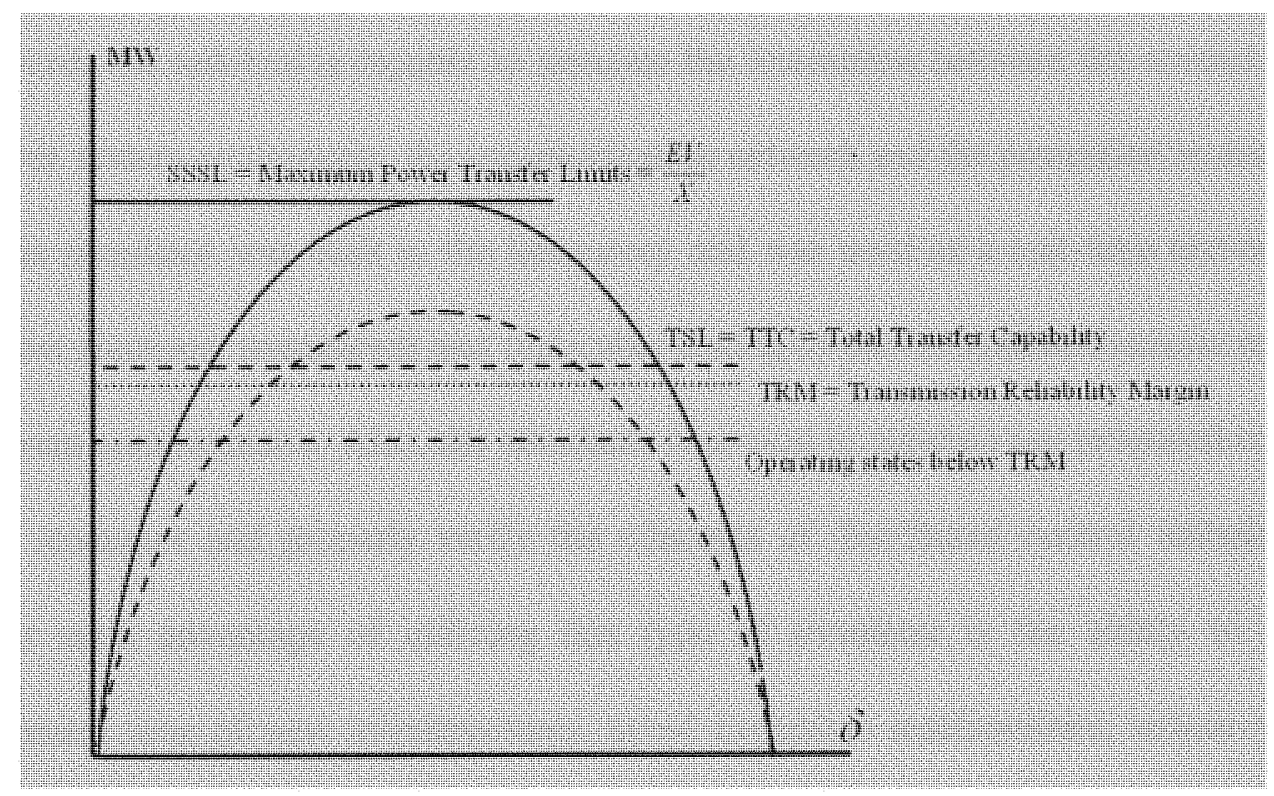

Figure (A.1) TTC and TRM seem from the steady-state stability perspective.

According to NERC [22], TTC is given by:

$$
\text { TTC }=\text { Min \{Thermal Limit, Voltage Limit, Stability Limit }\}
$$

Where thermal violation, voltage violation and frequency oscillations can be identified through on-line assessment and combined with off-line stability assessment for scheduled systems loading and other possible case scenario. (e.i. unscheduled inter-area transactions). 


\section{APPENDIX B}

\section{Description of the Test-Bed Elements}

This appendix reviews the details on the design and development of the test-bed power system simulator. The details of the equipments and components utilized for the development of the simulator are also described. Also, this appendix outlines the systematic methodology followed to achieve the experimental results.

\section{Test-Bed Power System Simulator}

The test-bed power system simulator was developed with the aim to emulate the interactions between the electrical network main subsystems in a real-time environment. In other words the test-bed is capable to simulate the interaction between the control system, the power system equipments, the communication network and the protection system.

This simulator was built in the laboratory and was used as a real-time test-bed. This will allow rigorous, transparent and replicable testing of power system dynamic responses to different contingencies. Also, the simulator was used to validate our proposed corrective control strategies and properly verify the concepts of distributed control infrastructures. Another main advantage of utilizing this type of simulator is the ability to improve development time of new infrastructures, avoid expensive malfunctions, reduce cost, and rigorously test the system before its implementation and eventual utilization in an operational system.

\section{Layout of the Overall Test-Bed}

The layout of the overall test-bed power system simulator is showing in Figure (B.2.1). This figure also shows the physical configuration of the test-bed including the utilized equipment and components already available in the laboratory. This configuration contains 
nine buses; six are represented by the sets of bus-bar models named B_1, B_2, B_3, B_4, B_5 and B_6. The other three remaining buses are represented by the nodes (Node_1, Node 2, Node 3) between the generators and the step-up transformers (not showed in the figure). Each of the bus-bar models is monitored and controlled by a remote terminal unit (RTU); these PCs are fitted with high-speed data acquisition cards.

A set of three motor-generator set-ups were utilized for the configuration $\left(G_{-}\right.$1, G_2, G_3); this set-up consist of a DC motor attached to a synchronous alternator through a shaft. The DC motor is utilized as the prime mover for the synchronous alternator, the DC motor is a two pole, compound wound rotor machine with a series field, shunt and armature winding. This machine is rated at 125 -Volts, 3.5 -Amps, $1 / 3$-hp at 1800 -rpms and shut-filed rated at 0.5 -Amps at 125 -Volts. The synchronous alternator is a three-phase, $1 / 3$-hp, rated at 1750 -rpms, and 208 line-to-line voltage at $60 \mathrm{~Hz}$. The machine is mounted behind a terminal box which consists of two ports for field connection to the rotor and six ports for three-phase connection to the stator. It also has six circuit breaker terminals and a circuit breaker switch. A toggle switch enables the user to switch the field connection for induction starting or synchronous run.

The six transmission lines (T-L_1, T-L_2, T-L_3, T-L_4 $\left., T-L_{-} 5, T_{-} L_{-} 6\right)$ shown in the configuration are represented by the transmission line models developed.

Three load-boxes (Load_1, Load_2, Load_3) were utilized to represent the loads in the simulator. The resistance-reactance load device has three variables, resistance, capacitance and inductance. The value of the resistance is controlled by six toggle switches each of which adds 2000-ohms in parallel with each other. The reactance loads are controlled by related knobs which serve to adjust the power factor of the load for either lagging 
(inductive) or leading (capacitive). The entire load may be used as single or three-phase applications for purely resistive, purely reactive or combined loads.

Also, the system simulator contains two Thyristor Switch Braking Resistor models (TSBR_1, TSBR); as previously mentioned in Chapter 5 are utilized as autonomous agents to prove some concepts on distributed control infrastructure for the power system. The TSBR model consists of one step-down three-phase transformer, one power electronic converter board and one resistive load-cart. The high side of the transformer is connected at the generator node. The low side of the transformer is connected to the power electronic converter board. The power electronic converter board consists of two independent three phase inverters. Each 3-phase PWM converters uses power MOSFETs as switching devices. The load-cart is utilized as the braking resistor. Each TSBR device is controlled by a real-time station (RTS). This PC utilizes a real-time operating system that allows realtime control over the power electronic converter board.

The entire simulator is controlled from the Master Station. This work-station PC is equipped with a high-speed processor that allows the processing of large amount of data. The Master Station collects data from each RTU. It also has full control over the operation of each RTU. This master/slave configuration emulates the SCADA environment in a central control infrastructure. Due to the high processing capacity of the Master Station, it is also utilized as the system coordinator for both RTS. This coordinator is responsible for the collaborative protocols and data sharing between both TSBR devices. The communication between the Master Station and RTSs; as well as, RTUs is done through two independent communication networks. The communication network utilized TCP/IP protocols. Two communication buses are included in the system (C-Bus_1, C-Bus_2). 


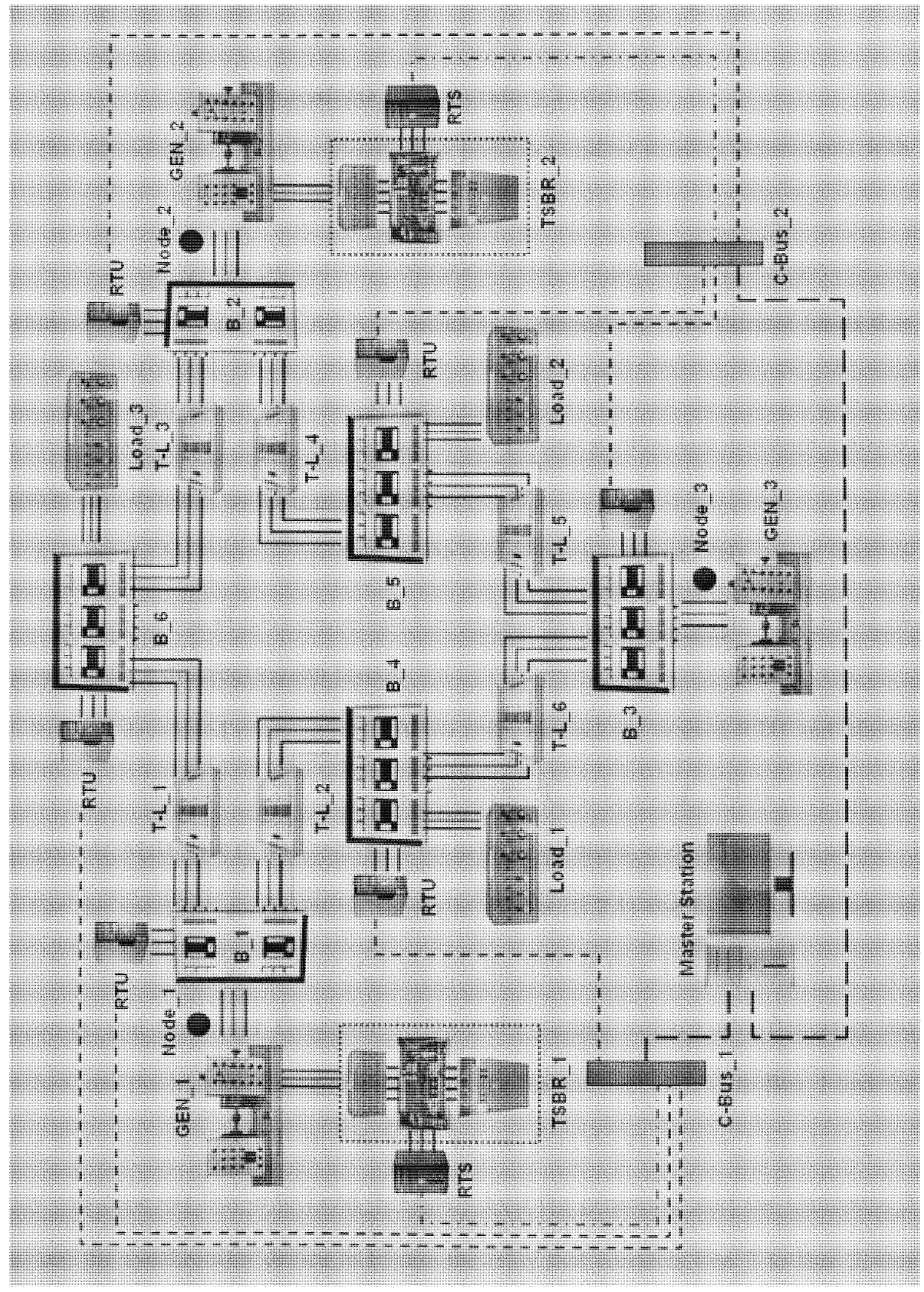

Figure (B.2.1) Physical configuration of the test-bed power system simulator. 
APPENDIX C

\section{Procedures for Laboratory Test-Bed}

The following steps can be identified to perform transient stability experiments with distributed control implementation on the hardware test-bed power system simulator.

Select power system parameters, components and ratings; this step is important for technical and safety reasons. All equipments and components have thermal limits that should never be reached for the steady state operation. All components and equipments can handle a physical limit violation for small periods of time (i.e. transient stability experiments, dynamic stability experiments).

Arrange the hardware components to the desired system layout. This step is possible due to the flexibility of the components blocks. In other words, the system can easily be rearranged for a different system layout.

Run the developed program in LabView software package at each RTU and Master Station; this step allows the SCADA environment to be setup before running the equipments. Make sure that all relays are set in the open mode, and that all loads are off.

For the specified configuration shown in Figure (B.2.1) the following procedures were developed. Start the Generator_1 and use the RTU at Bus_1 to monitor the voltage, frequency and phase for Generator_1. Once the desired voltage and frequency are reached, use the Master Station to close the relay that connects line_1 to Bus 1 and the relay that connects line_1 to Bus_6. Now, we can load the Generator_1 by closing the relay that connects Bus_6 to Load_3. Lightly load the generator, start the Generator_2 and use the synchronizer device to bypass the relay that connects line_3 to Bus_2; this procedure allows removing the synchronizer device after synchronization. Close the relay 
that connects line_3 to Bus_6. Now use the RTU at Bus_2 to read the voltage at Bus_2. At this point the synchronizer will flash until the phase, voltage and frequency of the generator matches the phase, voltage and frequency of Bus_2. As the frequency of the machine approaches that of the line, the frequency of the "flashing" will decrease. As the phase approaches that of the line, the lamps will become dim. At the instant the three lamps are all dark, the synchronizing conditions are met. Now, the relay that connects line_3 to Bus_2 can be safely closed. At this point Generator_1 and Generator_2 are sharing the load and working in synchronism. Now, close the following set of relays that connects:

- Line_2 to Bus_ 1 and Bus_4;

- Line 4 to Bus 2 and Bus_5;

- Line 5 to Bus_3 and Bus_5;

- Line 6 to Bus_3 and Bus_4;

At this point all RTUs and Master Station should be reading the bus voltages and some current absorbed by the transmission line models. Now, close the relays that connect Load_2 to Bus_5 and Load_1 to Bus_4. At this point the system can be slowly loaded to the desired point; this loading process should be done in steps of $2000 \mathrm{ohms}$ every 5 seconds and carefully adjusting the power outputs of the generators when needed. This allows the generator to reach the new steady state operation point before the next increase of load. Now, connect the synchronizer in series to the line between Node_ 3 and Bus_3 and close the relay that connects the synchronizer to Bus_3. Start Generator_3 and adjust the phase, voltage and frequency. At the instant the three lamps are all dark, the 
synchronizing conditions are met and the synchronizing switch can be closed safely. At this point, the three generators are sharing the load and working in synchronism.

Once the desired loading conditions are met and the system is operating in steady state mode, the two FACTS devices can be turned-on. Run the control program, developed on RT-Lab software package on the two RTS PCs from the Master Station.

Now the system is ready for experimental applications. The following experiments are suggested:

- Three-phase fault at any line;

- Fault at a generator bus and load bus;

- Small changes in load by adding or removing loads in steps of $2000 \mathrm{ohms;}$

- Abrupt changes in load by adding motors or removing large loads;

In order to study the results, the system is designed to keep records of the events if desired. This feature is initiated from the Master Station with the following options:

- Choose which parameter at which location is desired to be recorded;

- Choose where the information should be stored (i.e. Master Station or any RTU);

- Choose how the data should be recorded (i.e. time scale, sampling scale, format, etc)

- Choose the data to be printed or exported to a data base program or system historian (i.e. Microsoft Excel, Microsoft Access); 
APPENDIX D

\section{Design and Development of Transmission Line and Bus Models}

\section{D-1 Transmission Line Models}

The test-bed power system simulator is a three phase system simulator rated at 2-k VA, 208-V . This simulator was originally designed to model the scaled up IEEE 9-bus test system. Here, the system was scaled down by using the voltage transformer VT's and the current transformer (CT's) ratios as in equation (B-4.1).

$$
Z_{\mathrm{sec}}=\frac{R_{\mathrm{CT}} * Z_{\mathrm{pri}}}{R_{\mathrm{vT}}}
$$

Where $Z_{\text {sec }}$ is the impedance in the secondary side in ohms that represents the scaled impedance using the VT's and CT's turns ratio, $Z_{\text {pri }}$ is the impedance of the primary in ohms. Here, $R_{V T}$ and $R_{C T}$ are the VT turns ratio and $C T$ current ratio, respectively. This method allows the rapid scaling of the system components such as transmission lines. Basically, the system is scaled referring to the low side of the voltage and current transformer. The following example is solved for a 55-km, 50-MVA, and 110-kV overhead transmission line with the following characteristics: Conductor type $=\mathrm{Cu} 150 \mathrm{~mm}^{2}$,

$$
\begin{aligned}
& \mathrm{R}_{\mathrm{L}}^{\prime}=0.119 \Omega / \mathrm{km} \quad \rightarrow \quad \mathrm{R}_{\mathrm{L}}=6.55 \Omega \\
& \mathrm{X}_{\mathrm{L}}^{\prime}=0.40 \Omega / \mathrm{km} \quad \rightarrow \quad \mathrm{X}_{\mathrm{L}}=22.0 \Omega \\
& \mathrm{C}_{\mathrm{L}}^{\prime}=9.2 \mathrm{nF} / \mathrm{km} \quad \rightarrow \quad \mathrm{C}_{\mathrm{L}}=509 \mathrm{nF}
\end{aligned}
$$

The maximum current must be define to select the correct CT ratio,

$$
I_{L}=\frac{50 * 10^{6}}{\sqrt{3} * 110^{*} 10^{3}}=263 \text { Amps }
$$


We can safely approximate the CT ratio and VT ratio to be,

$$
\mathrm{R}_{\mathrm{CT}}=\frac{200}{5}=40 \quad \mathrm{R}_{\mathrm{VT}}=\frac{110 \mathrm{kV}}{\sqrt{3} * 120}=529.2
$$

Now, the line resistance and reactance; as well as, the shunt reactance can be obtained,

$$
\begin{aligned}
& \mathrm{R}_{\mathrm{L}}=\frac{6.55 * 40}{529.2}=0.495 \Omega \\
& \mathrm{X}_{\mathrm{L}}=\frac{22.0 * 40}{529.2}=1.66 \Omega \\
& \mathrm{X}_{\mathrm{C}}=\frac{6.3 * 10^{3} * 40}{529.2}=476.19 \Omega
\end{aligned}
$$

The above values correspond to the scaled transmission line; these values were utilized to design the $\pi$-model shown in Figures (B.4.1). These values were utilized to choose the components for the transmission line model shown in Figure (B.4.2).

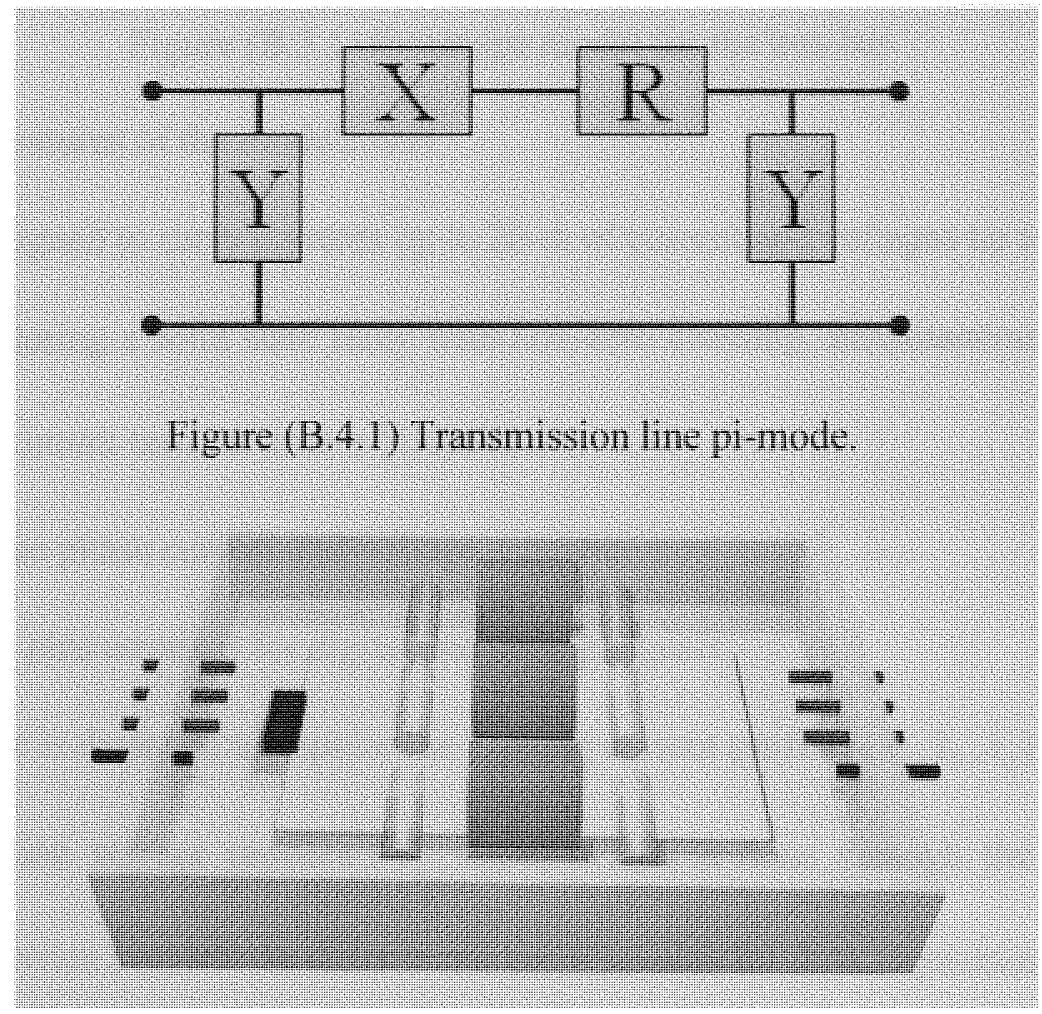

Figure (B.4.2) Scaled transmission line model. 


\section{D-2 Design and Development of the Bus-Bar Model}

The bus-bar model consists of a module that contains switching devices, measurement devices (sensors) and data acquisition (DAQ) terminals. This module is then interfaced to a PC through a data acquisition card from National Instruments. The PC uses LabView software package for data display, assessment and control and it is acting as a Remote Terminal Unit (RTU). Figure (B.5.1) shows the prototype model for the bus-bar.

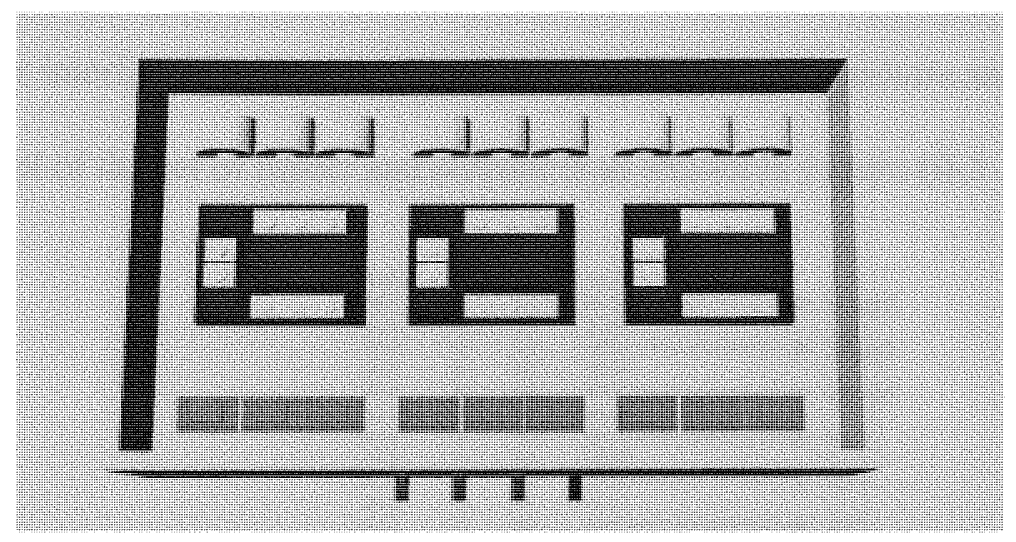

Figure (B.5.1) Prototype model for the bus-bar.

The main features of the module are:

- Designed to carry currents up to 20-A of current and voltages up to 530-V;

- Featuring a solid state relay with non-zero crossing, instantaneous turn on. The maximum turn-on time $=1 / 2$ cycle;

- Three phase current and voltage sensors;

- 100 pins terminal for digital and analog signals;

The bus-bar is designed to capture the 3-phase current profiles $\left(I_{a}, I_{b}, I_{c}\right)$ and the threephase line to line voltage profiles $\left(\mathrm{V}_{\mathrm{a}}, \mathrm{V}_{\mathrm{b}}, \mathrm{V}_{\mathrm{c}}\right)$. This is done through the utilization of voltages and current sensors. In order to use these sensors for data acquisition system, finetuning was required. The utilization of a $100-\Omega$ multi-turn potentiometer was necessary to 
achieve the required tuning. Figure (B.5.2) shows the circuit schematics for the sensor components.

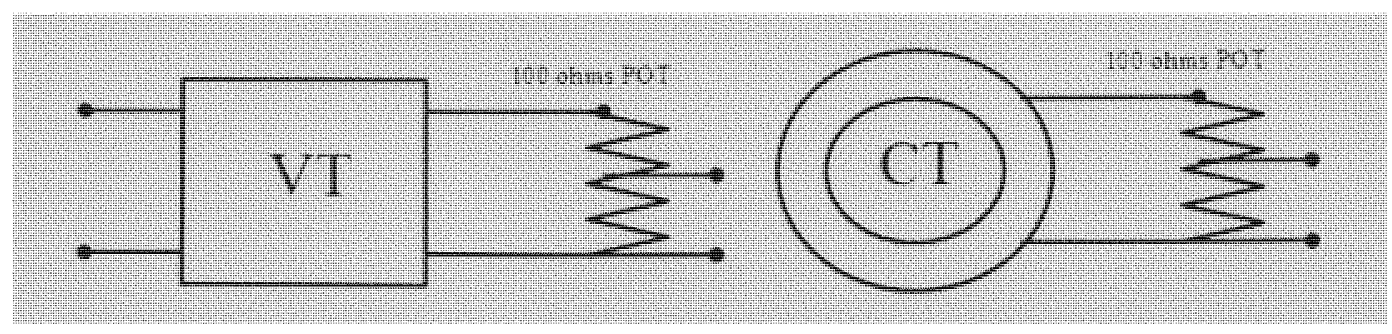

Figure (B.5.2) Circuit schematics for the sensor components.

The computer interface was done by creating two fifty-pins connectors and determining the pin assignment of the set of inputs and outputs is as shown in Figure (B.5.3).

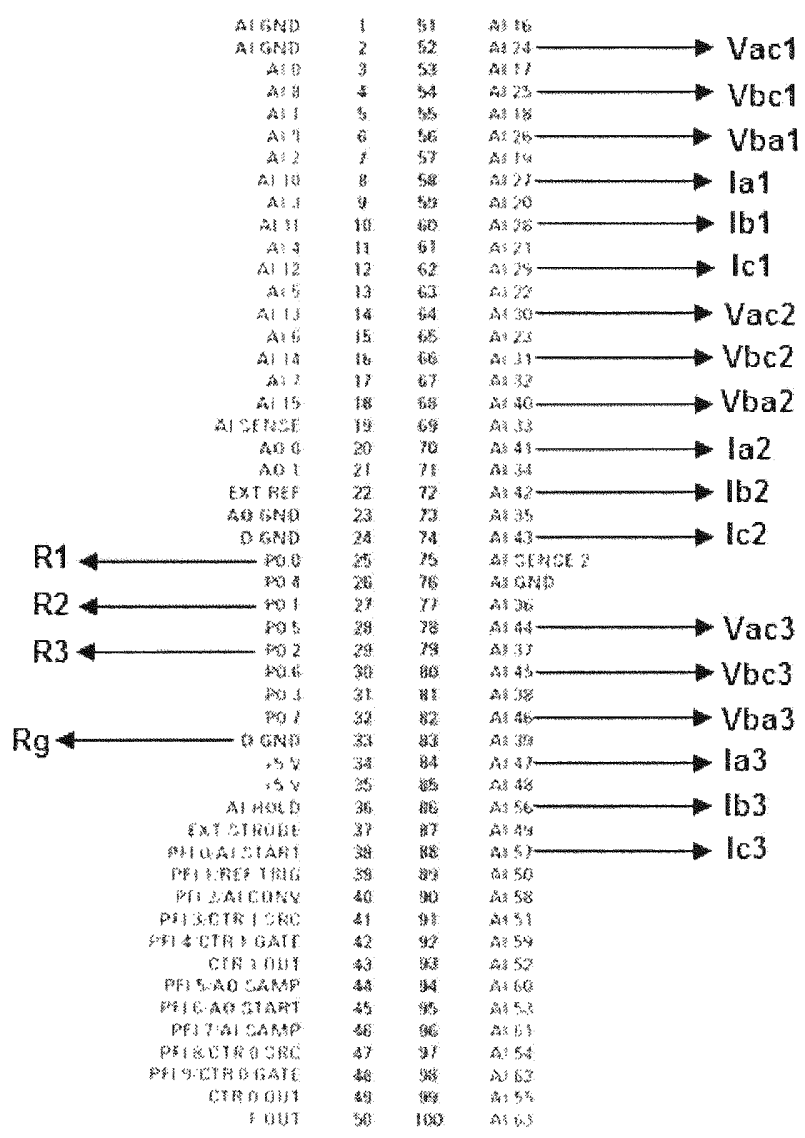

Figure (B.5.3) Pin assignment.

Further details can be found in the laboratory manual. 